Historic, Archive Document

Do not assume content reflects current scientific knowledge, policies, or practices. 

63.1

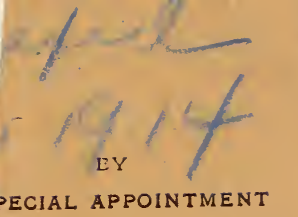

OPECIAL APPOINTMENT

62

\section{HARDY ORNAMENTAL \\ TREES AND SHRUBS}
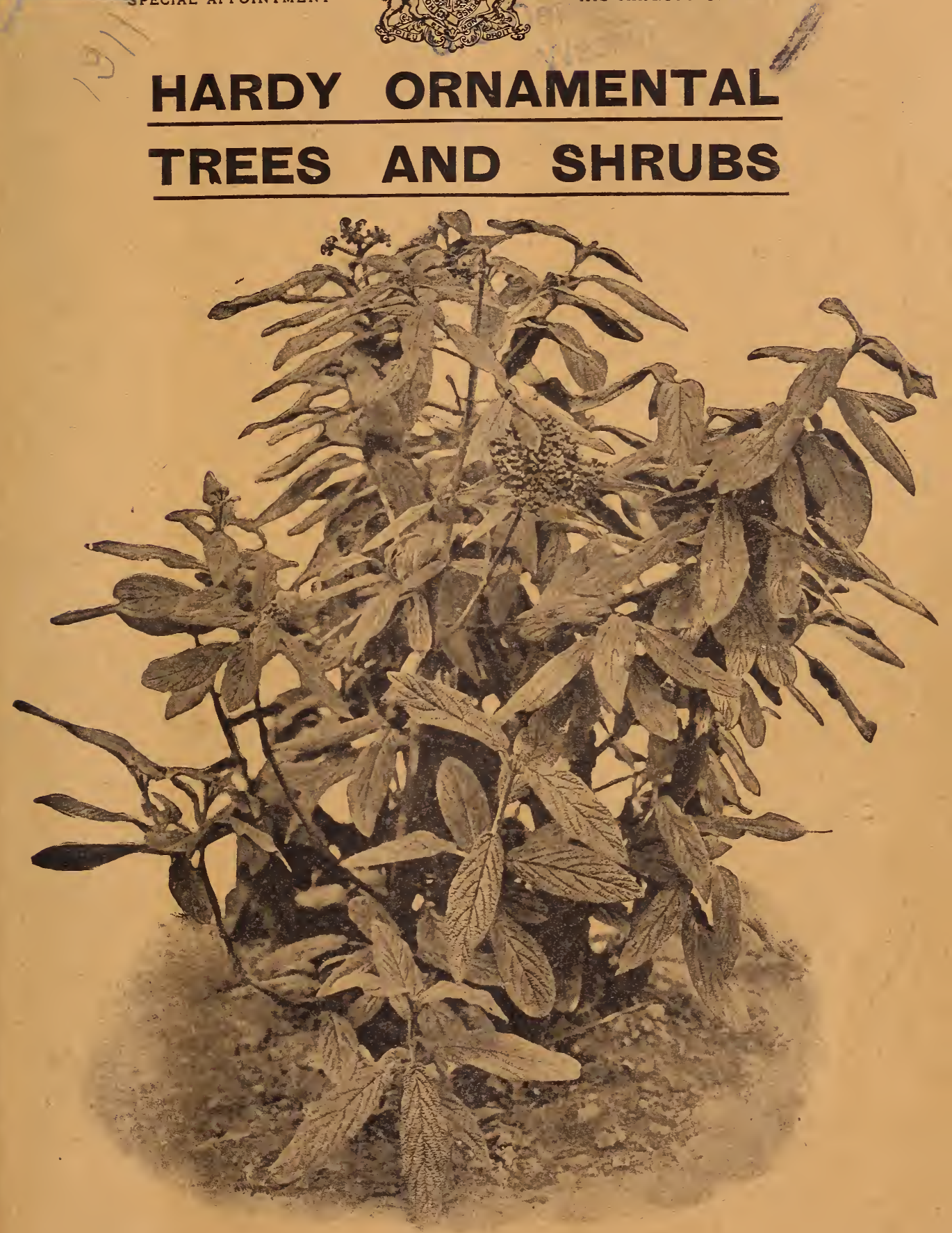

VIBURNUM RHYTIDOPHYLLUM.

See page 34

\section{JAMES VEITCH \& SONS CHELSEA}

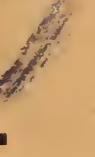




\section{VISITORS}

will be cordially welcomed at all our Nurseries on all week days except Bank Holidays.

The various Branches are as follows:

Royal Exotic Nursery,

544, King's Road,

Chelsea, London.

Telephone: 1642, Western.

Telegrams : Veitch, Chelsea.

Coombe Wood, Kingston Hill.

Station-NORBITON

(L. \& 8. W. R., Kingston Line)

Cabs at the Station

\section{Langley, near Slough}

Station-SLOUGH (G.W.R.)

$(1$ mile to Langley

New Town Entrance)

Cabs at the Station

\section{Feltham, Middlesex}

Station-FELTHAM (L. \& S. W. R., Windsor and Reading Line)

10 Minutes walk
Vegetable and Flower Seeds, Bulbs, and all Garden Tools and Sundries. Stove and Greenhouse Plants, Orchids, Palms, Ferns, Bay Trees in tubs, etc. All requirements for London Houses and Gardens.

\author{
Hardy Ornamental Trees \\ and Shrubs, \\ Avenue Trees, Yews, \\ American Plants, Climbers, \\ Rhododendrons, Conifers, Bamboos, \\ Hardy Water Lilies and \\ Aquatic Plants, etc. \\ Roses in Pots
}

Apple, Pear and Cherry Trees, Currants and Gooseberries, Strawberries, Hardy Herbaceous

Perennials and Alpine Plants, Roses in the open ground, Choice Seed Cultures

\section{SPECIALITY.}

A magnificent stock of Fruit Trees in pots for the Orchard House.

Trained Peaches, Nectarines, Apricots, Plums, Cherries, Apples, Pears, etc., Grape Vines and Fig Trees in pots, Raspberries, - Asparagus, Seakale, Rhubarb, etc.

Begonias, Carnations, Chrysanthemums, Camellias, Dahlias, Gloxinias, Fuchsias, etc. Seed Trial Grounds. 


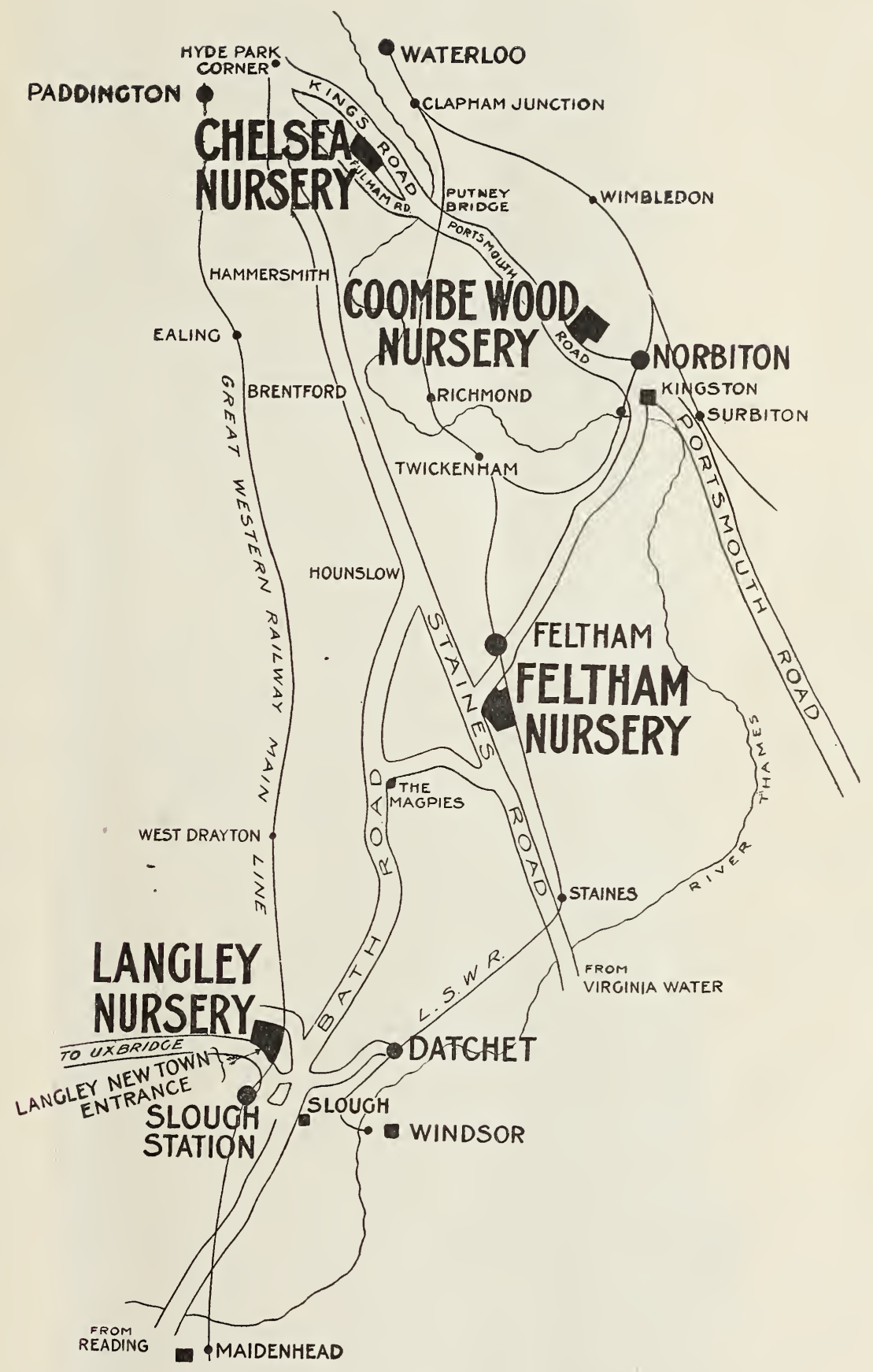

Sketch Map showing the positions of Veitch's Nurseries. 


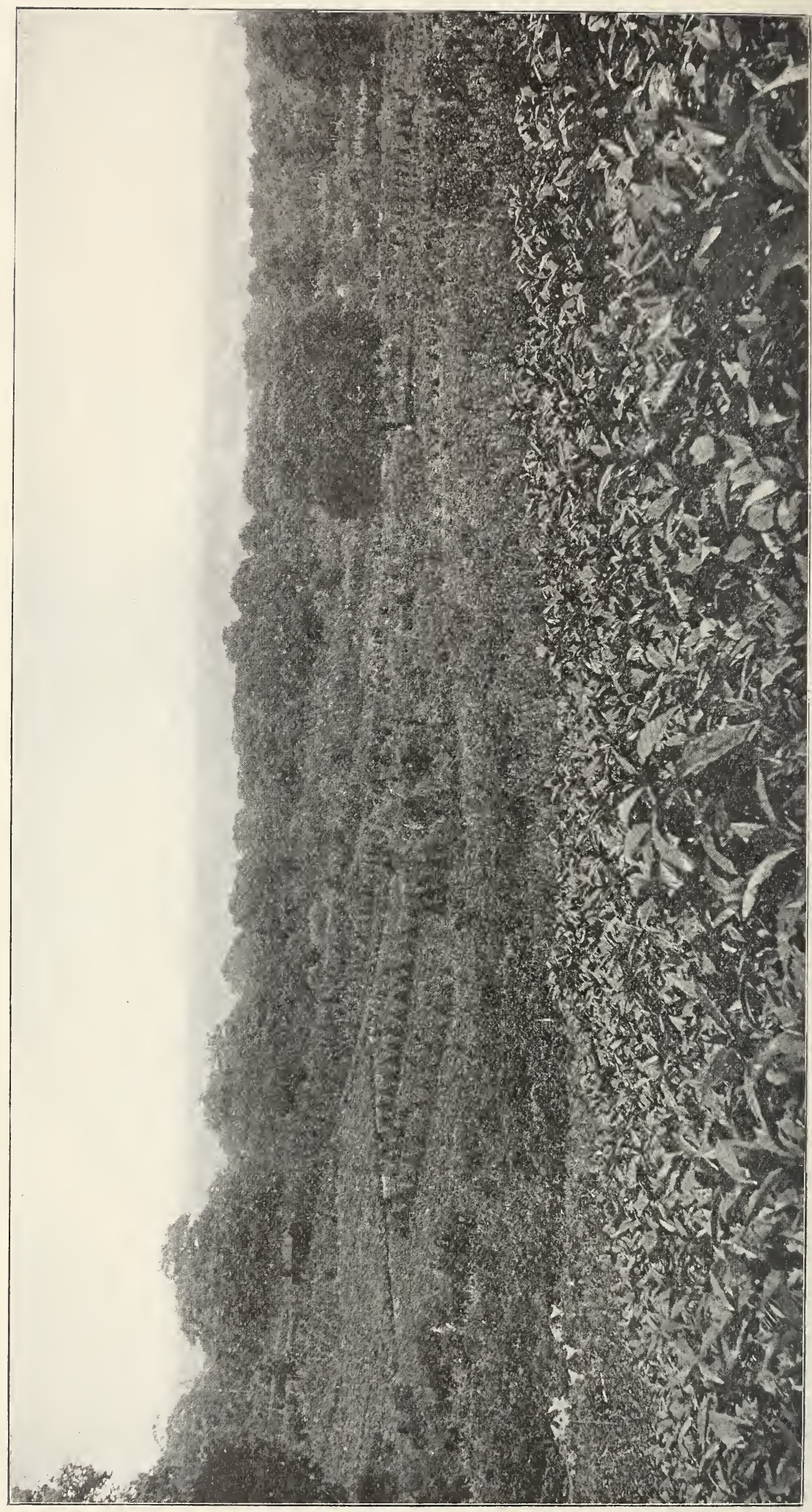


All interested in beautiful Trees and Shrubs are most cordially invited to visit the Coombe Wood Nursery, which faces the Portsmouth Road on the top of Kingston Hill. The route from the West End of London lies through Putney and Kingston Vale. The nearest railway station is Norbiton, on the Kingston branch of the London and South Western Railway, but the Nursery may also be reached from Kingston and Surbiton Stations, the latter being on the same Company's main line. Cabs are always in waiting at all three stations. Trams run from Surbiton to within five minutes' walk of the Nursery.

Prices.

The lowest prices in this Catalogue are usually for ordinary sizes, but we shall be very pleased to forward on application quotations for specimen plants or large quantities.

Good Results depend on how plants have been grown. Our plants have been frequently transplanted, which ensures good results for our customers provided the plants receive proper treatment after they leave our Nurseries.

Orders by Post receive the best attention and customers unable to make à personal call may rely on obtaining plants selected with the greatest care.

Export Orders may be entrusted to us with complete confidence as we are accustomed to send our plants, suitably packed, to all parts of the world.

Cash Discount at Five per cent is allowed on invoices of the value of $20 /-$ or over, if paid within 30 days from date of invoice.

This discount does not apply to export orders, as the value is made up to the customer in extra strong plants, specially selected to stand the voyage.

This discount also does not apply to special quotations and estimates, which are always strictly nett.

Gratis Plants to help towards the cost of carriage, are added whenever the order is sufficiently large.

Packing Charges are reduced to lowest cost, consistent with the well-known high standard of our packing, which ensures plants and trees reaching our customers in the best possible condition. The charges include the cost of all material as well as the skilled labour of our special staff's of packers. Half-price will be allowed on empty crates, boxes and baskets if returned at once in good condition, carriage paid and advised by post. Bundles are not returnable.

$$
\text { JAMES VEITCH \& SONS, }
$$




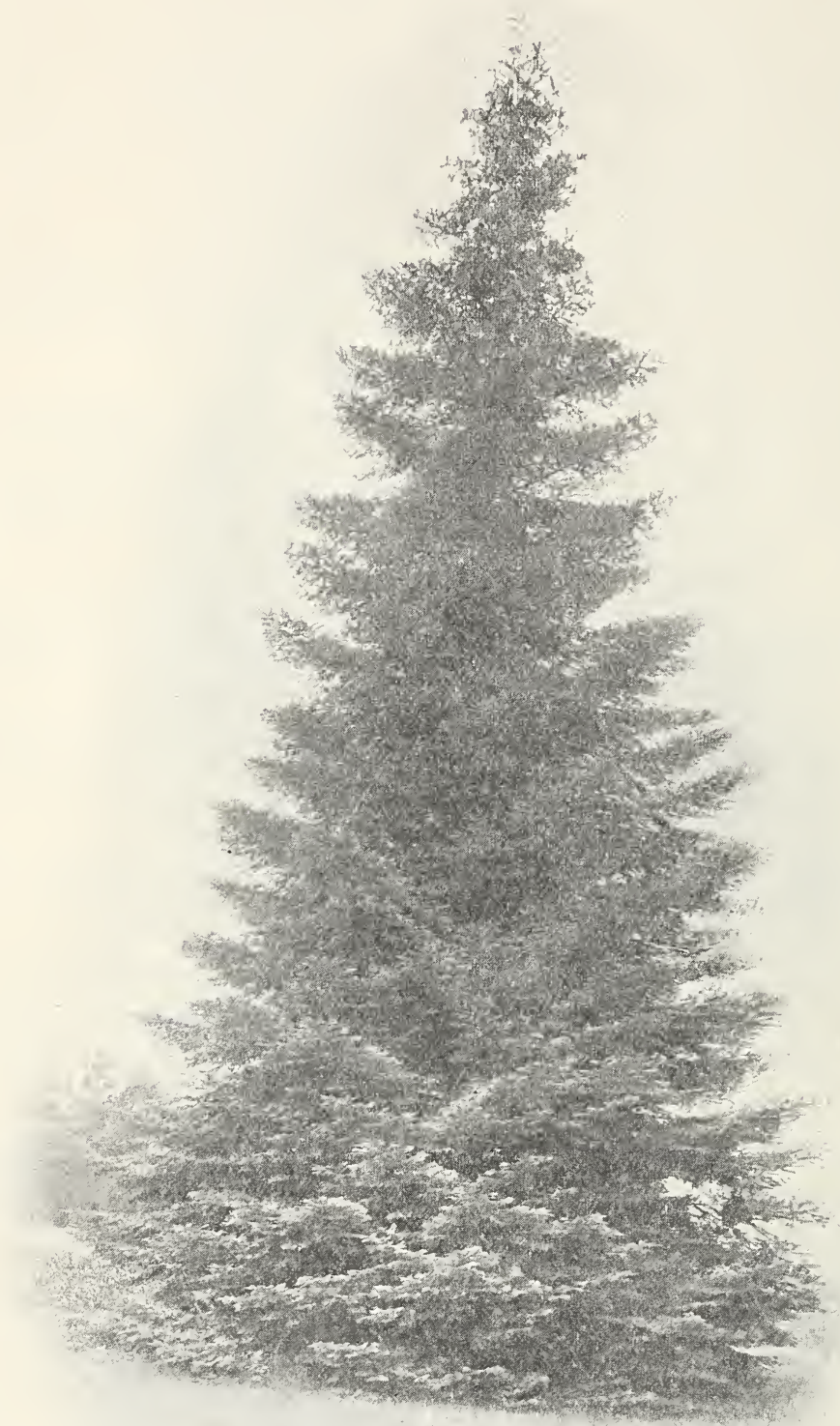

Abies grandis (Gordoniana).

A Silver Fir of rapid growth, symmetrical habit and rich green foliage (see page 5) 


\section{CONIFERS.}

\section{GENERAL COLLECTION.}

\begin{tabular}{|c|c|c|c|}
\hline NAME. & NATIVE COLXTRY. & PRICE PER & PLANT. \\
\hline \multirow{10}{*}{$\begin{array}{l}\text { Abies. } \\
\text { ajanensis (Picea) } \\
\text { alba } \\
\text { Albertiana (Tsuga) } \\
\text { - aurea , } \\
\text { Alcockiana (Picea) } \\
\text { brachyphylla (homolepis) } \\
\text { bracteata } \\
\text { canadensis (Hemlock Spruce) } \\
\text { - argentea (Tsuga) }\end{array}$} & & & \\
\hline & Japan & $3 / 6$ to & $10 / 6$ \\
\hline & North America & $1 /-$ & $3 / 6$ \\
\hline & British Columbia & $2 / 6$ & $5 /-$ \\
\hline & Garden variety & $3 / 6$ & $5 /-$ \\
\hline & Japan & - & $5 /-$ \\
\hline & Japan & $2 / 6$ & $10 / 6$ \\
\hline & S. California & - & $5 /-$ \\
\hline & N. America & $1 / 6$ & $5 /-$ \\
\hline & Gardeu variety & $3 / 6$ & $5 /-$ \\
\hline -parvifolia ," &,$\quad$, & 36 & $5 /-$ \\
\hline - pendula , & , & $3 / 6$ & $7 / 6$ \\
\hline cephalonica (Apollinis) & Greece & $2 / 6$ & $10 / 6$ \\
\hline cilicica & Asia Minor & $3 / 6$ & $5 /-$ \\
\hline concolor & Colorado & $3 / 6$ & $21 /-$ \\
\hline - Lowiana (lasiocarpa) & California & $3 / 6$ & $21 /-$ \\
\hline Douglasii (Pseudotsuga) & N. W. America & $1 / 6$ & $\mathbf{2 1} /-$ \\
\hline - glauca (Tsuga) & Garden variety & $2 / 6$ & $21 /-$ \\
\hline Engelmannii (Picea) & Rocky Mountains & $2 / 6$ & $\mathbf{5} / \cdot$ \\
\hline - glauca & Garden variety & $3 / 6$ & $5 /-$ \\
\hline excelsa (Norway Spruce) , & Europe & -16 & $5 /-$ \\
\hline - clanbrassiliana & Garden variety & $2 / 6$ & $5 /-$ \\
\hline - conica , & ,, & $2 / 6$ & $3 / 6$ \\
\hline - finedonensis (rariegated) & ," & $2 / 6$ & $10 / 6$ \\
\hline - Gregoriana $\quad$, & , & $2 / 6$ & $\mathbf{5} / \cdot$ \\
\hline - inverta (weeping) & , & $3 / 6$ & $7 / 6$ \\
\hline - mutabilis & , & $2 / 6$ & $5 /-$ \\
\hline -pumila & ," & $2 / 6$ & $5 /-$ \\
\hline - pygmæa &., & $2 / 6$ & $5 /-$ \\
\hline - Remontii & , & $2 / 6$ & $5 /-$ \\
\hline firma & Japan & $3 / 6$ & $7 / 6$ \\
\hline Fraseri & North Carolina & $2 / 6$ & $5 /-$ \\
\hline Glehnii (Picea) & Sachalin and Yesso & $3 / 6$ & $5 /-$ \\
\hline $\begin{array}{l}\text { grandis (Gordoniana) } \\
(\text { see illustration } \text { p. 4) }\end{array}$ & Oregon and Vancourer's Island & $2 / 6$ & $7 / 6$ \\
\hline Hookeriana (Tsuga) & N. W. America & $3 / 6$ & $21 /-$ \\
\hline lasiocarpa arizonica & $\begin{array}{l}\text { U. S. America. The silvery } \\
\text { foliage is of great beauty } \\
\text { the bark is very remark- } \\
\text { able, being cream-coloured } \\
\text { and of a corky nature }\end{array}$ & $5 /-$ & $10 / 6$ \\
\hline magnifica & California & $3 / 6$ & $10 / 6$ \\
\hline Mariesii & Northern Japan & $5 /-$ & $7 / 6$ \\
\hline Menziesii (Sitchensis) (Picea) & N. W. America & $1 / 6$ & $3 / 6$ \\
\hline nigra (Black Spruce) & North America & $1 / \cdot$ & $5 / \cdot$ \\
\hline nobilis & British Columbia and Oregon & $2 / 6$ & $10 / 6$ \\
\hline Nordmanniana & Caucasian Mountains & $2 / 6$ & $21 /$ \\
\hline numidica & Algeria, Atlas Mountains & $3 / 6$ & $10 / 6$ \\
\hline
\end{tabular}




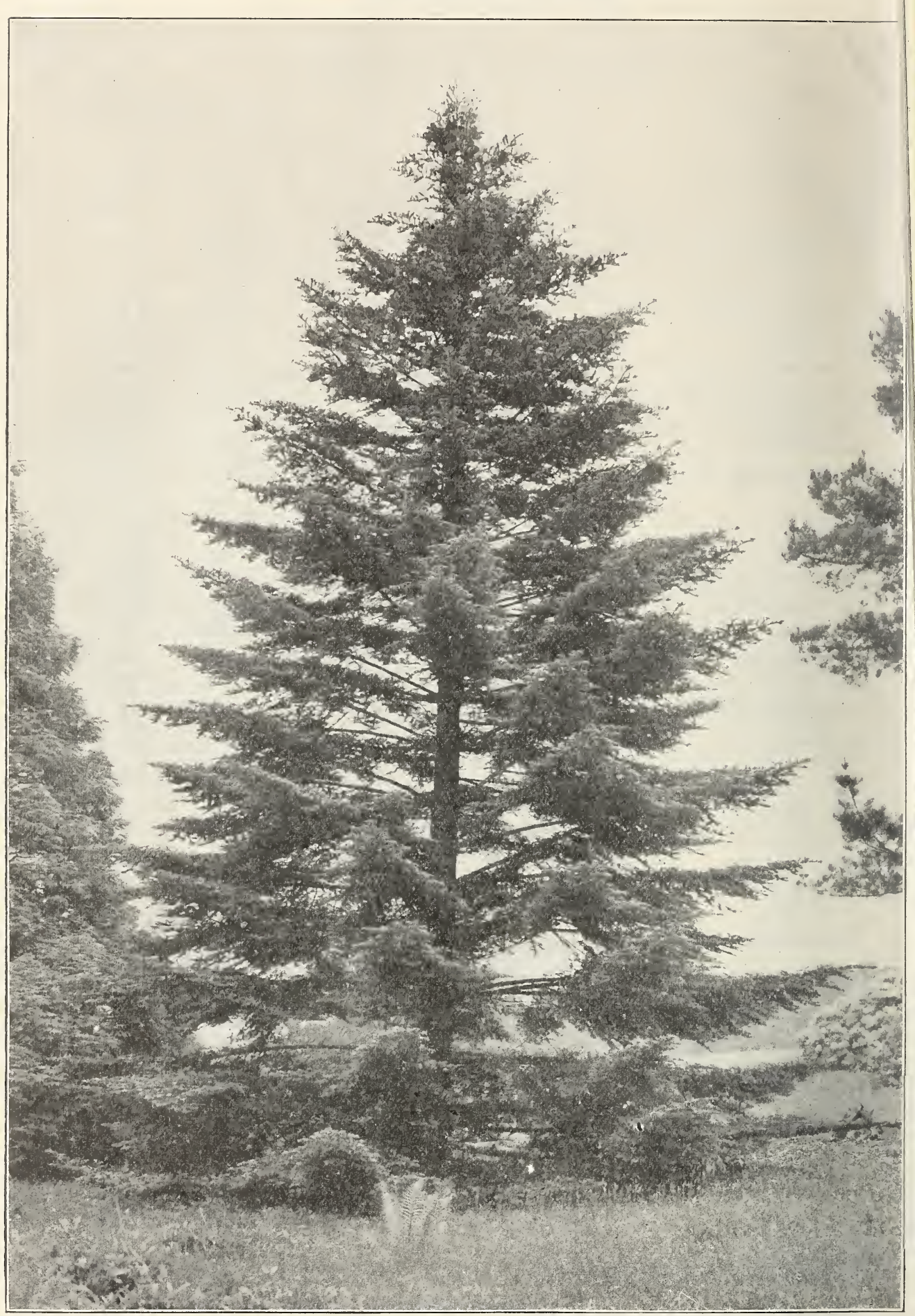

Abies Veitchii.

Slender habit, bright green and white foliage, and beautiful cones. An ornamental tree for lawns, etc. (see page 7 ). 


\begin{tabular}{|c|c|}
\hline \multicolumn{2}{|l|}{ NAME. } \\
\hline $\begin{array}{c}\text { Abies (continued). } \\
\text { obovata }\end{array}$ & (Picea) \\
\hline $\begin{array}{l}\text { Omorica (Servian Spru } \\
\text { orientalis }\end{array}$ & xe) , \\
\hline $\begin{array}{l}\text { Pattoniana glauca. } \\
\text { Pinsapo }\end{array}$ & eriana \\
\hline polita & (Picea) \\
\hline pungens (Parryana) & , \\
\hline - glauca (Blue Spruce) & , , \\
\hline - - pendula & , , \\
\hline
\end{tabular}

Sieboldii (Tsuga)

Smithiana (Picea)

subalpina (lasiocarpa, true)

Tsuga (Tsuga)

Veitchii (see illustration p. 6)

Araucaria (Chile Pine) imbricata

Athrotàxis (Tasmanian Cypress) cupressoides

Biota (Chinese Arbor Vitæ).

orientalis. See Thuia orientalis

Cedrus (Cedar). p. 14)

atlantica (africana)

- aurea

- glauca

\section{Deodara \\ - argentea \\ - aurea \\ - crassifolia \\ - robusta \\ - viridis}

Libani (Cedar of Lebanon)

Cephalotàxus (Chinese $\mathrm{Yew}$ ) drupacea

Fortunei

pedunculata

- fastigiata (Taxus japonica)

Chamacyparis. See Cupressus p. 8 Cryptomèria (Japanese Cedar) japonica (true)
NATIVE COUNTRY.

Siberia

S. E. Europe

Trans-Caucasia and Armenia

Spain

Japan

Colorado and Utah

Garden variety

Differing from the type in the colour of its bluish grey foliage and also in its pendulous branches and branchlets.

Japan

N. W. Himalaya

N. W. America

Japan

Japan

Andes of Valdivia, Chile

Tasmania

Algeria, Atlas Mountains

A variety in which the young foliage is a rich golden yellow.

An intensely glaucous variety; one of the most striking of park and landscape trees. Silvery white foliage appearing in the distance as if covered with hoar-frost.

N. W. Himalaya

Garden variety

,"

Syria

Japan

China

Japan

,

Japan
PRICE PER PLANT.

$3 / 6$ to $5 /$ -

$36 \quad 10 / 6$

$2 / 6 \quad 10 / 6$

2/6 21/.

$3 / 65 /$.

2/6 $7 / 6$

$3 / 6 \quad 21 /$.

$7 / 6 \quad 42 /-$

$3 / 65 /$ -

$3 / 6 \quad 5 /-$

$3 / 6 \quad 10 / 6$

$2 / 6 \quad 7 / 6$

$3 / 621 /$.

$3 / 6 \quad 21 /-$

$3 / 6 \quad 5 /-$

$1 / 621 /$.

5/. $\quad 21 /$.

$2 / 621 /$ -

2/6 21/.

$3 / 6 \quad 7 / 6$

$3 / 6 \quad 10 / 6$

$3 / 6 \quad 7 / 6$

$3 / 6 \quad 7 / 6$

$3 / 6 \quad 5 /-$

$3 / 6 \quad 10 / 6$

$3 / 6 \quad 7 / 6$

$3 / 6 \quad 5 /-$

- $3 / 6$

$2 / 6 \quad 5 /=$

$2 / 6 \quad 5 /=$ 


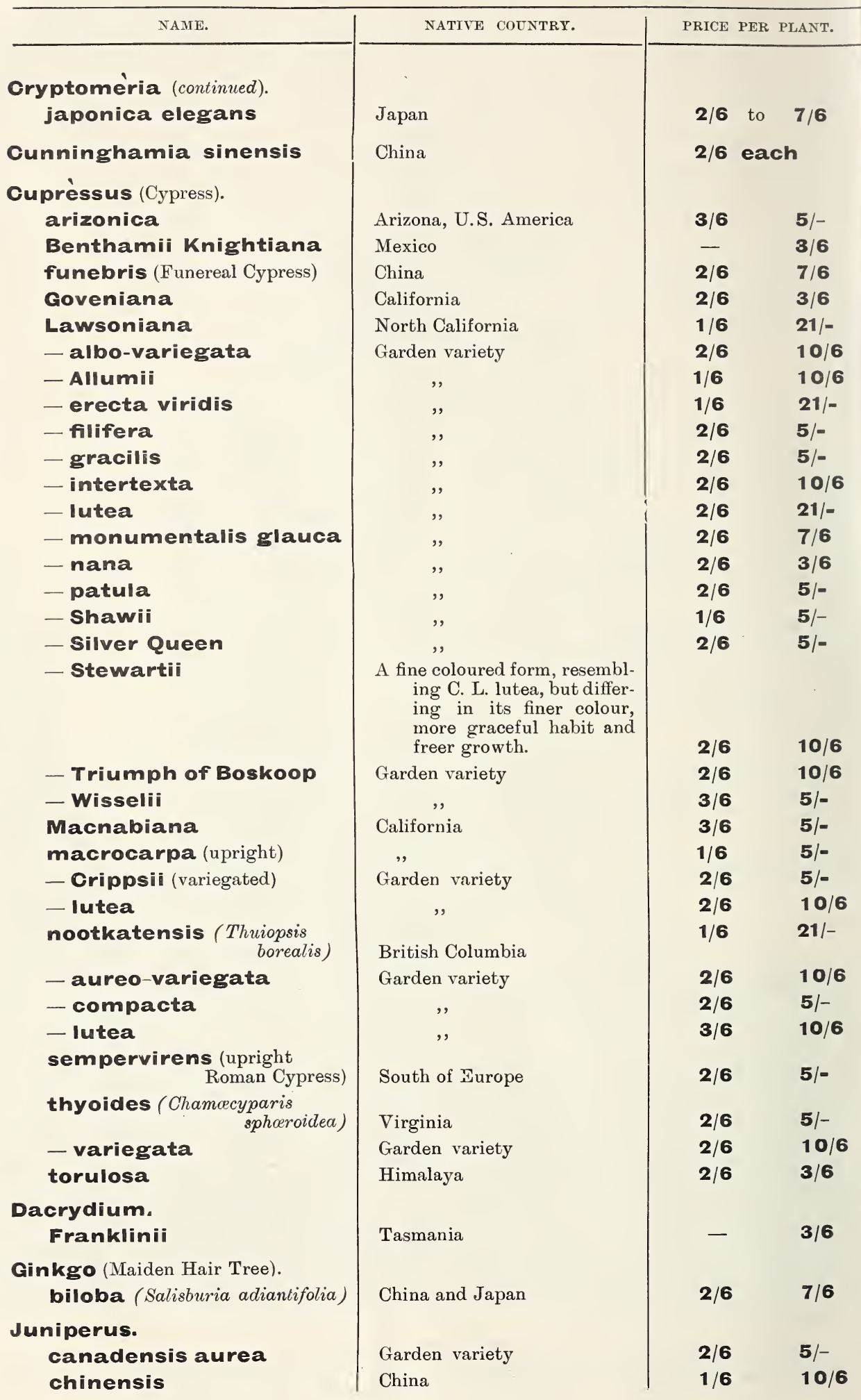




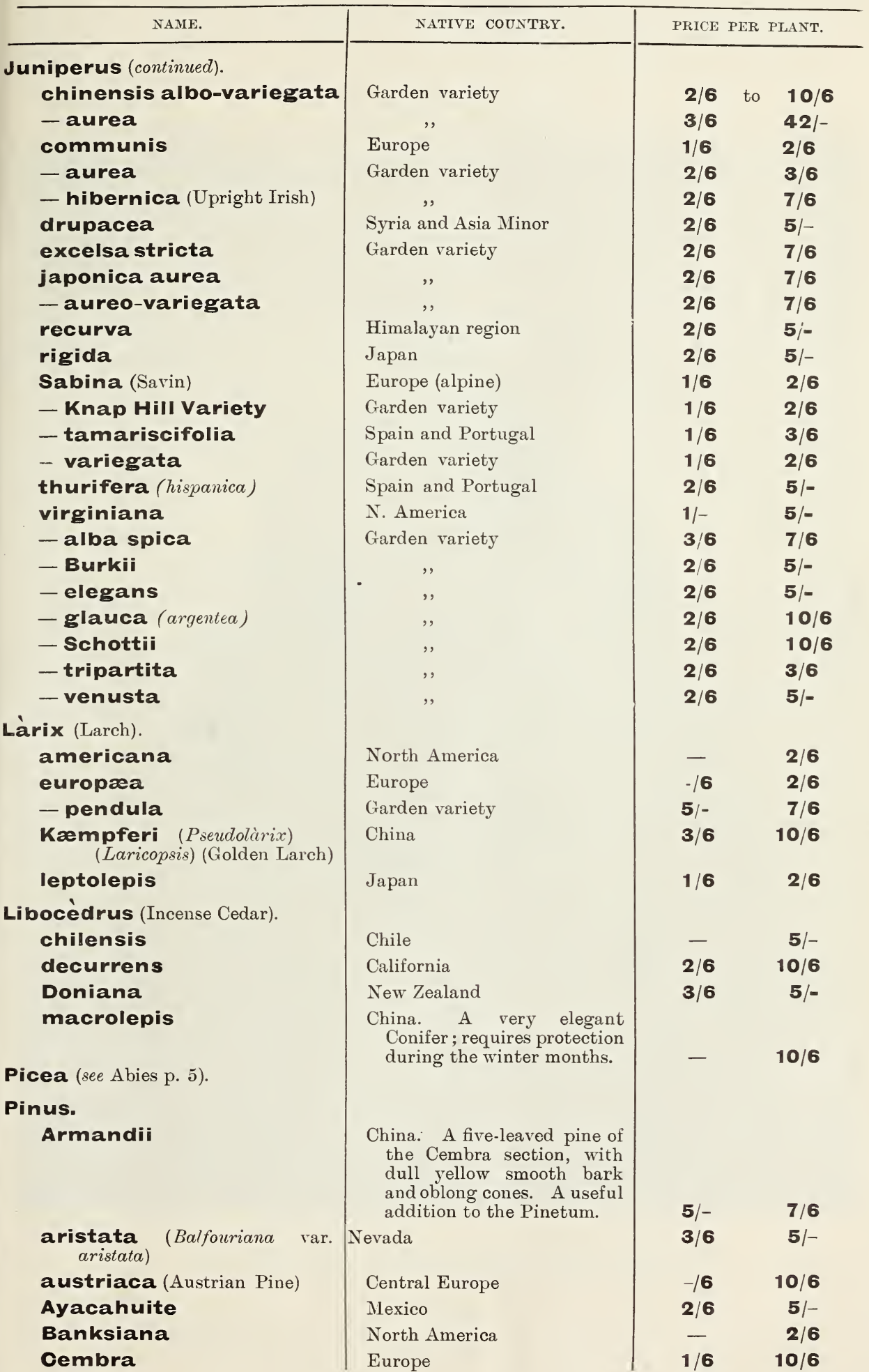




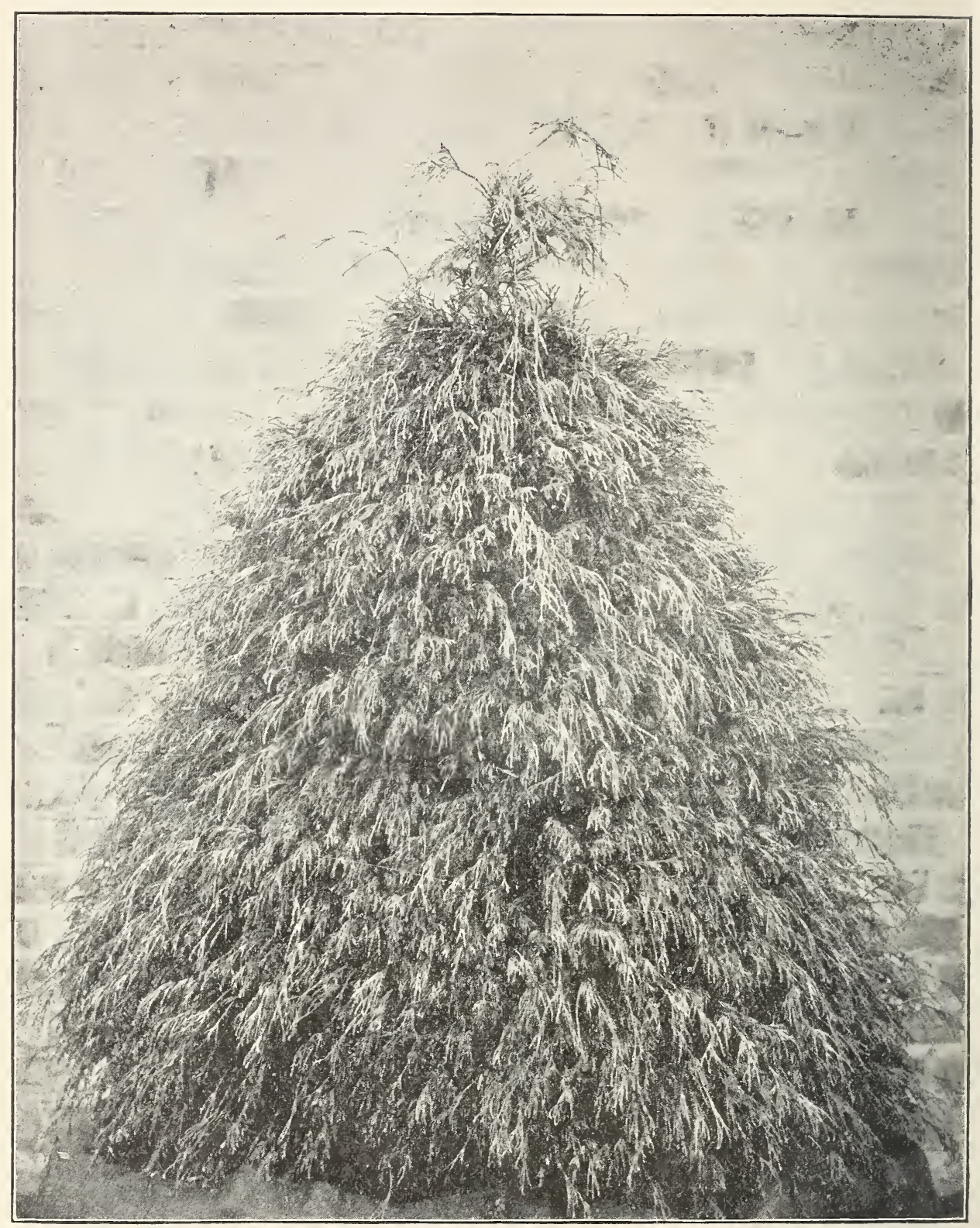

Retinispora filifera aurea (see page 11). 


\begin{tabular}{|c|c|c|c|c|}
\hline NAME. & NATIVE COUNTRY. & PRICE & PER & PLANT. \\
\hline \multicolumn{5}{|l|}{ Pinus (continued) } \\
\hline contorta & California and Oregon & $3 / 6$ & to & $5 / \cdot$ \\
\hline - Murrayana & N.W. United States & - & & $3 / 6$ \\
\hline Coulteri (marcrocarpa) & California & $2 / 6$ & & $5 /-$ \\
\hline densiflora & Japan & $1 / 6$ & & $3 / 6$ \\
\hline excelsa (Himalayan Pine) & Sikkim and Nepal & $1 / 6$ & & $5 /-$ \\
\hline flexilis & California & - & & $3 / 6$ \\
\hline inops (Scrub Pine) & Eastern United States & $2 / 6$ & & $3 / 6$ \\
\hline insignis (radiata) & California & $1 / 6$ & & $5 /-$ \\
\hline Jeffreyi & , & $2 / 6$ & & $3 / 6$ \\
\hline koraiensis & Northern China & $2 / 6$ & & $5 /-$ \\
\hline Lambertiana & California & $3 / 6$ & & $5 /-$ \\
\hline Laricio (Corsican Pine) & South of Europe & $1 /-$ & & $3 / 6$ \\
\hline monophylla (Fremontiana) & Sierra Nevada, Utah & - & & $3 / 6$ \\
\hline montana (Mugho) (Mountain & Alps and S. W. Europe & $1 /-$ & & $2 / 6$ \\
\hline monticola & North California & $2 / 6$ & & $5 /-$ \\
\hline parviflora & Japan & $2 / 6$ & & $5 /-$ \\
\hline ponderosa & N.W. United States & $2 / 6$ & & $3 / 6$ \\
\hline Pinaster (maritima) (Cluster & South of Europe & $1 /-$ & & $2 / 6$ \\
\hline pinea (Stone Pine) & Italy & $\mathbf{1} /-$ & & $2 / 6$ \\
\hline rigida (Pitch Pine) & Eastern United States & $2 / 6$ & & $3 / 6$ \\
\hline Sabiniana (Nut Pine) & California & $2 / 6$ & & $3 / 6$ \\
\hline Strobus (Weymouth Pine) & North America & -16 & & $3 / 6$ \\
\hline - nana & Garden variety & $2 / 6$ & & $5 /-$ \\
\hline sylvestris (Scotch Pine) & North of Europe & -16 & & $5 /-$ \\
\hline - argentea & Garden variety & $2 / 6$ & & $3 / 6$ \\
\hline - nana & ,, & $2 / 6$ & & $5 /-$ \\
\hline Thunbergii (Massoniana) & Japan & $2 / 6$ & & $3 / 6$ \\
\hline \multicolumn{5}{|l|}{ Podocàrpus. } \\
\hline alpinus & Tasmania & - & & $5 /-$ \\
\hline chilinus & Chile & $2 / 6$ & & $3 / 6$ \\
\hline macrophyllus & Japan & - & & $5 /-$ \\
\hline Totara & New Zealand & $2 / 6$ & & $\mathbf{5} /-$ \\
\hline $\begin{array}{l}\text { Prumnópitys (Stachycarpus). } \\
\text { elegans }\end{array}$ & Southern Chile & $2 / 6$ & & $10 / 6$ \\
\hline \multicolumn{5}{|l|}{ Pseudolárix. See Larix p. 9.} \\
\hline \multicolumn{5}{|l|}{ Pseudotsuga. See Abies p. 5} \\
\hline \multicolumn{5}{|l|}{ Retinispora (Cupressus). } \\
\hline $\begin{array}{c}\text { filicoides (var. of Cupressus } \\
\text { obtusa) }\end{array}$ & Japan & $2 / 6$ & & $7 / 6$ \\
\hline - tetragona aurea & Cxarden variety & $2 / 6$ & & $10 / 6$ \\
\hline$\underset{\text { pisifera) }}{\text { filifera }}$ (var. of Cupressus & Japan & $2 / 6$ & & $21 /-$ \\
\hline - aurea (see illustration p. 10) & Garden variety & $3 / 6$ & & $10 / 6$ \\
\hline leptoclada & , & $2 / 6$ & & $\mathbf{5} /-$ \\
\hline Cupressus obtusa) & Japan & $2 / 6$ & & $10 / 6$ \\
\hline obtusa & , & $2 / 6$ & & $7 / 6$ \\
\hline
\end{tabular}




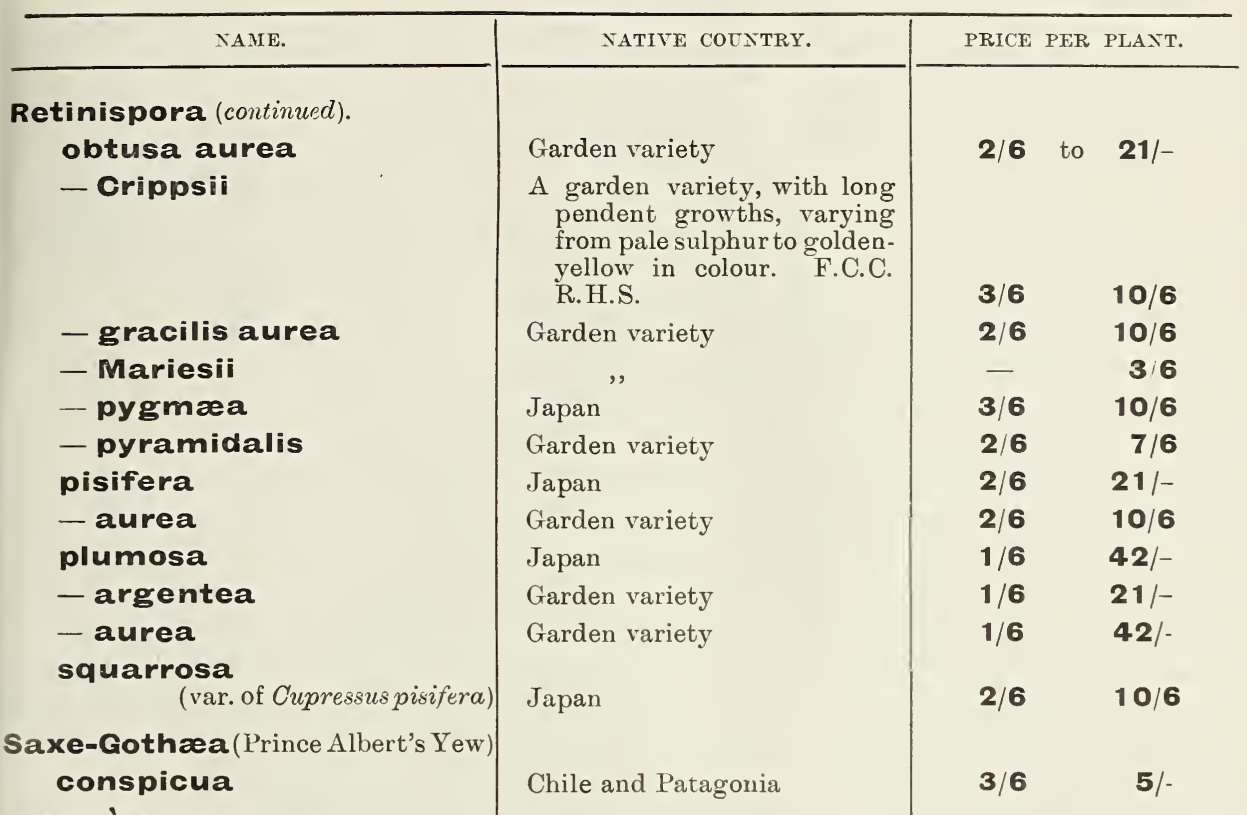

Sciadópitys (Umbrella Pine). verticillata (see illustration p. 15)

\section{Sequòia. \\ gigantea, see Wellingtonia gigantea $p .14$. sempervirens \\ (Californian Redwood) (Taxodium sempervirens)}

- alba spica

Taxódium (Deciduous Cypress). distichum

- pendulum

sempervirens See Sequoia above

Taxus (Yew).

baccata (English Yew)

\section{- adpressa}

- - stricta

- aurea

- Dovastonii (weeping)

- elegantissima

- erecta

$\cdot$

Japan. A rery distinct Conifer, and also one of the most remarkable evergreen trees ever introduced.

\section{-}

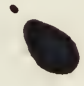

California

Garden variety

N. America, Atlantic States

Europe and Asia

We have an exceptionally fine stock of Yews specially grown for hedge planting, and we shall be pleased to give quotations for quantities on application.

Garden variety

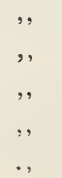

$\begin{array}{rr}2 / 6 & 7 / 6 \\ 2 / 6 & 10 / 6 \\ 2 / 6 & 42 /- \\ 3 / 6 & 21 /- \\ 2 / 6 & 42 /- \\ 2 / 6 & 21 /-\end{array}$




\begin{tabular}{l} 
NAME. \\
\hline Taxus (continued). \\
baccata erecta aurea \\
- fastigiata (Upright Irish) \\
- - argenteo-variegata \\
- - aureo-variegata \\
- fructu-Iuteo \\
- Washingtonii \\
cuspidata \\
japonica. See Cephalotaxus p. 7
\end{tabular}

Thuia (Arbor Vitæ).

dolabrata (see illustration p. 12)

- Iatevirens (nana)

- variegata

gigantea (Thuia Lobbii)

- aurea

\section{- pyramidalis}

japonica (Standishii) occidentalis

(American Arbor Vitæ)

- elegantissima

(George Peabody)

- Ellwangeriana

- Späthii

- Vervaeneana

- Wareana

- aurea

orientalis

- aurea (Golden Arbor Vitæ)

- elegantissima

Thuiópsis.

\section{borealis}

See Cupressus nootkatensis p. 8 dolabrata. See Thuia dolabrata Tórreya (Fetid Yew).

californica (myristica)

\section{grandis}

nucifera

Tsuga. See Abies p. 5.

Wellingtónia (Sequoia). gigantea

(7)

NATIVE COUNTRY.

PRICE PER PLANT.

This variety has the whole of the current season's growth, rich golden yellow; it is quite distinct from the golden varieties of the common and Irish Yews.

Garden variety

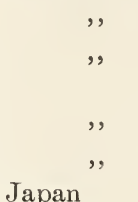

Japan

Japan

,

,

British Columbia

The value of $T$. gigantea for hedge planting cannot be overstated, and we shall be pleased to supply large quantities specially grown. Sizes and prices on application.

Garden variety

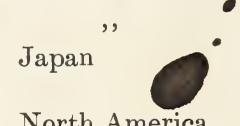

North America

Garden variety

,,

Garden variety

,

China

Garden variety ,

$\begin{array}{rr}2 / 6 & 42 /- \\ 2 / 6 & 5 /- \\ 2 / 6 & 21 /- \\ 1 / 6 & 21 / .\end{array}$

$2 / 6$ to $10 / 6$

$1 / 621 /$ -

$2 / 6 \quad 21 /-$

$3 / 6 \quad 21 /$.

$2 / 6 \quad 5 /-$

$2 / 6 \quad 5 /-$

$2 / 6 \quad 5 /-$

$3 / 6 \quad 5 /-$

- $2 / 6$

$3 / 6 \quad 5 /-$

$1 / 6 \quad 5 /-$

$2 / 6 \quad 7 / 6$

$1 / 6 \quad 2 / 6$

$2 / 6 \quad 3 / 6$

$2 / 6 \quad 10 / 6$

$1 / 6 \quad 7 / 6$

$2 / 6 \quad 5 /-$

$2 / 6 \quad 10 / 6$

$2 / 6 \quad 5 /$.

$3 / 6 \quad 5 /-$

California

North China

Japan

$\begin{array}{ll}3 / 6 & 7 / 6 \\ - & 5 /- \\ 3 / 6 & 5 /-\end{array}$

Sierra Nevada, California 


\section{HARDY RHODODENDRONS.}

Rhododendrons and their allies grow best in bog soil or in peat-mould, but when this cannot be procured, a good fertile loam with a dressing of rather fresh cow-manure, once in two years, forms a good substitute. When neither of these soils is available, a good mixture may be made as follows:-To three parts of halfrotted leaves add one part of the turfy surface of a pasture, cut about 4 inches thick; to this add a good quantity of white or other sand; chop but do not beat the soil, and use it as rough as possible. Should the foliage become yellowish, top-dress with cow-manure, or use liquid manure about four times during the month of July; by these means the plants will soon be restored to health. The seed pods should be carefully removed immediately after the plants have flowered.

In preparing beds or ground for Rhododendrons, the sub-soil should be sufficiently porous or drained to prevent water becoming stagnant beneath and around the roots of the plants. As these plants do not root deeply, from 4 to 6 inches of the soil beneath the balls will be sufficient, with a top-dressing annually added.

\section{RHODODENDRONS IN COLLECTIONS.}

To those unacquainted with the different varieties, we shall be pleased to give the benefit of our extensive experience in making a selection, on being informed of the number of plants required, the amount intended to be expended, together with the situation in which they are to be planted, and the prevailing colours required. By this plan a better selection can generally be made, and at a less cost than when the kinds are chosen otherwise.

Fine plants from the various sections will be supplied at the following prices when the selection is left to ourselves :-

All the best older garden varieties Per dozen.

Per 100.

Very fine newer sorts

$24 /$ - to $42 /$ $150 /$ - to $250 /$ -

Ponticum, good strong plants for

planting in woods, etc.

$30 /-$ to $84 /$

Ponticum, larger

Unnamed hybrids

Early-flowering varieties, suitable

for forcing and conservatory

decoration

$42 /-, 63 /-$ to $84 /-$

$12 /$ - to $42 /-$

$18 /$ - to $42 /-150 /-\&$ upwards

$30 /-$ to $42 /-$

\section{STANDARD AND HALF-STANDARD RHODODENDRONS}

Of all the leading kinds can be supplied at moderate prices; also some extra fine specimens. Particulars on application. 


\section{RHODODENDRONS}

\section{GARDEN HYBRIDS AND VARIETIES}

Containing all the finest and most distinct English and Continental varieties. Very hardy, of free growth, and with good foliage.

The prices quoted are for good established plants; many can be supplied in larger plants at proportionate prices.

\section{GENERAL COLLECTION.}

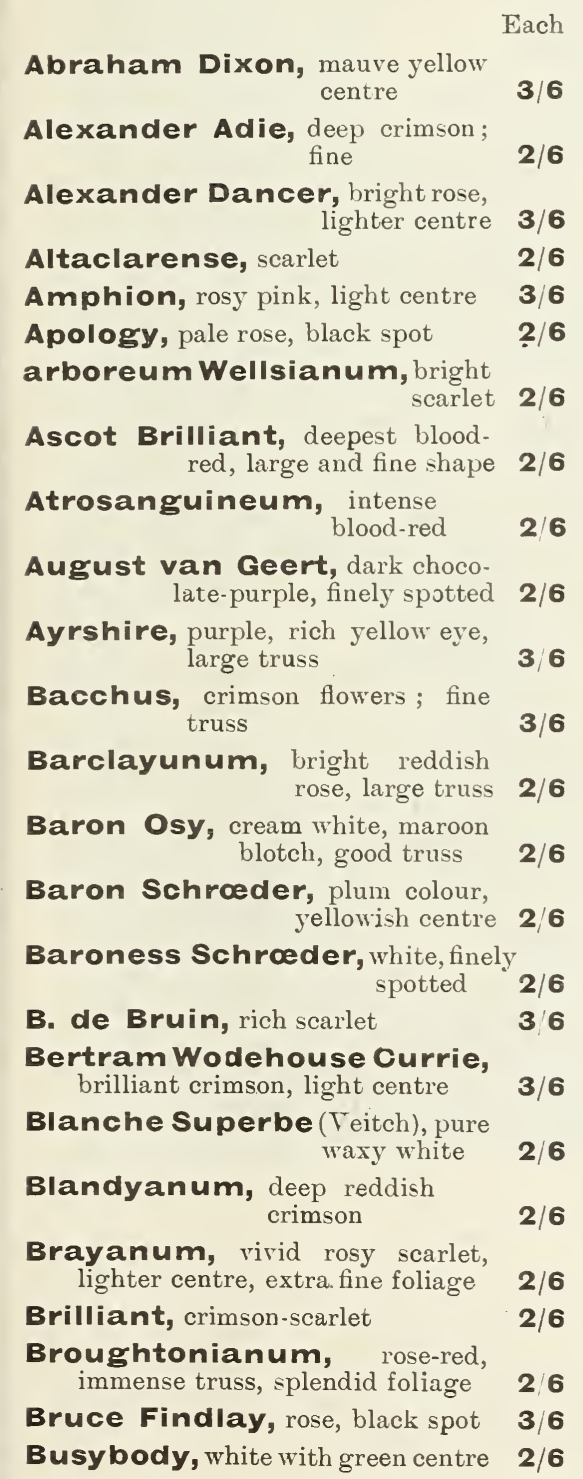

Each

Caractacus, rich purplish crimson, good truss

Catawbiense album elegans, waxy white, yellow spots 2/6

- purpureum elegans, very fine purple $\mathbf{3} / \mathbf{6}$

- grandifiorum, purple, large flowers $\quad 3 / 6$

- roseum elegans, a favourite old variety $\mathbf{2} / \mathbf{6}$

Caucasicum album (Cunningham's Dwarf White) 2/6

Caucasicum pictum, pale pink, lighter centre 2/6

Charles Bagley, cherry-red, large truss

$2 / 6$

Charles Dickens, dark scarlet

$2 / 6$

Charles Thorold, purple, greenish yellow centre 2/6

Charlie Waterer, scarlet, light centre

$3 / 6$

Concessum, deep rose-pink, paler centre

2/6

Countess of Clancarty, light rose-crimson, prettily marked 2/6

C. S. Sargent, bright scarlet, excellent habit and foliage delicatissimum, delicate blush, changing to white $2 / 6$

Doncaster, brilliant scarlet; one of the best of its colour $\mathbf{3 / 6}$

Duchess of Bedford, crimson, light centre 2/6

Duchess of Connaught, white with lemon-yellow markings 2/6

Duchess of Edinburgh, crimson, lighter centre

Duke of Connaught, rosecrimson, lighter centre 2/6

Earl of Shannon, deep crimson 2/6 Edward S. Rand, bright scarlet 2/6

Everestianum, rosy lilac, spotted and fringed 


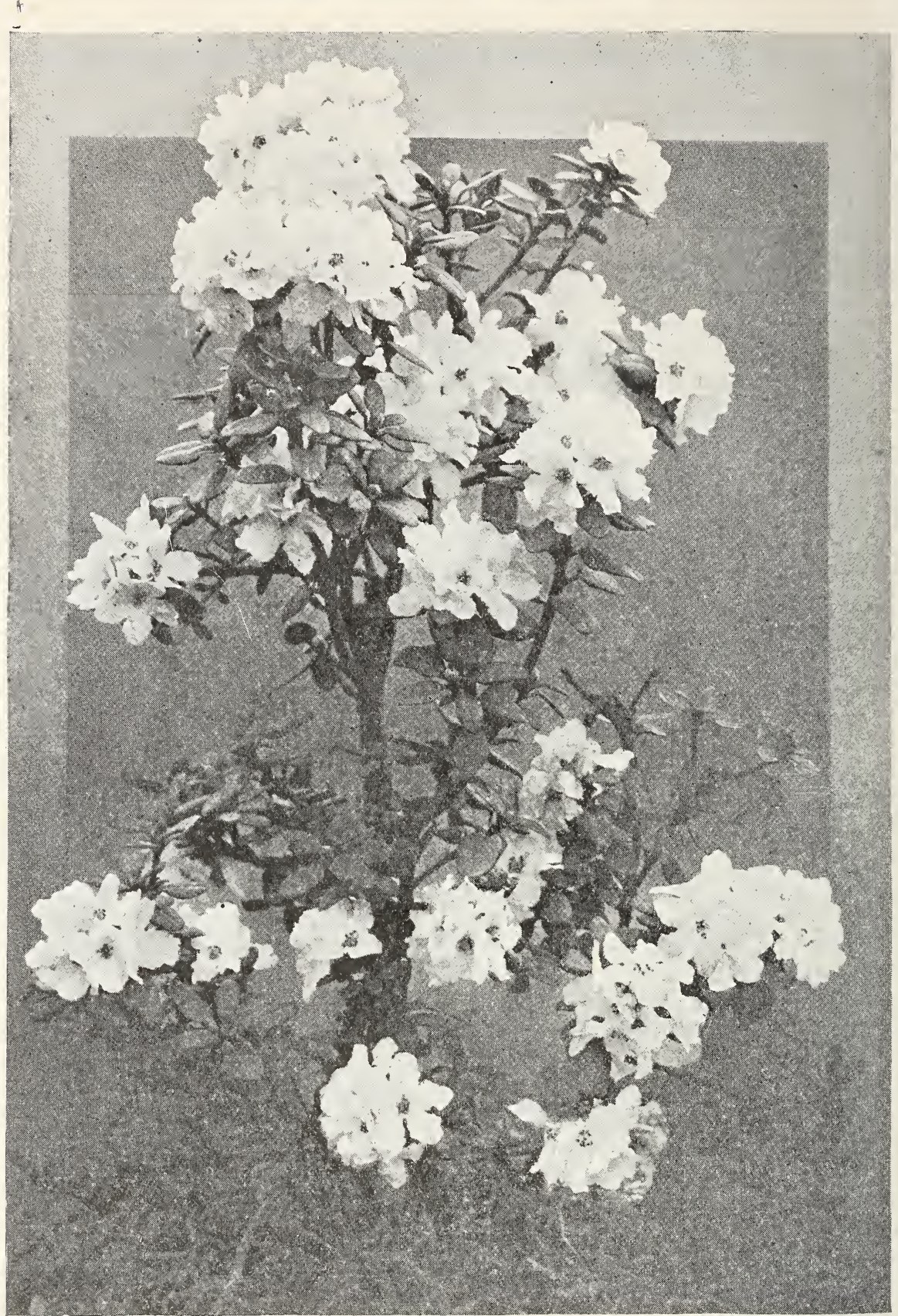

Rhododendron intricatum.

Lavender-coloured flowers, about $\frac{1}{2}$-inch in diameter, borne in trusses in great profusion. This species is dwarf, very compact and a charming suloject for the alpine garden; quite distinct from any other Rhododendron in cultivation (see page 22). First Class Certificate from the Royal Horticultural Society.

$$
3 / 6 \text { each }
$$




\section{GENERAL COLLECTION-continued.}

Fair Helen, white with yellow spots

Fastuosum, fi. pl., mauve; large trusses of double flowers $2 / 6 \&$

F. D. Godman, crimson blotched

Florence, pink with lighter centre

Florence Smith, pink, very distinct

Francis B. Hayes, white, black spots

Francis Dickson, brilliant scarlet 2/6

Frederick Waterer, fiery crimson, fine truss $2 / 6 \&$

George Hardy, pinkish white

George Paul, crimson, finely spotted

Giganteum, smooth, bright rosepink, very large truss

Gomer Waterer, white

Guido, deep crimson

Handsworth Early Red, very bright rose

Helen Waterer, pure white with bright crimson margin 2/6 \&

Helen Schiffner, pure white

Henrietta Sargent, pink, fine foliage

H. H. Hunnewell, rich crimson

H. W. Sargent, crimson, large truss

James Bateman, clear rosy scarlet

James McIntosh, rose-crimson James Marshall Brooks, rich scarlet with brown spots

James Mason, pale crimson centre, deeper towards the edge

James Nasmyth, rose-lilac with an intense maroon blotch

John Henry Agnew, pale blush, chocolate spots

John Spencer, rose with deep pink margin ; a late bloomer

John Walter, rich crimson $2 / 6$ \& John Waterer, deep glowing crimson, free bloomer

Joseph Whitworth, dark purplelake, dark spots

Kate Waterer, rose, yellow centre Kettledrum, rich purplish crimson King of the Purples, distinct in colour, of fine habit

Lady Annette de Trafford, cream colour with chocolate blotch $2 / 6 \&$

Lady Armstrong, pale rose, much spotted
Each

2/6

$3 / 6$

$2 / 6$

$3 / 6$

$3 / 6$

$5 /=$

$2 / 6$

$2 / 6$

$5 /-$

$2 / 6$

$2 / 6$

$3 / 6$

$3 / 6$

$3 / 6$

$3 / 6$

$3 / 6$

$2 / 6$

$2 / 6$

$2 / 6$

$2 / 6$

2/6

$2 / 6$

$3 / 6$

2/6

$2 / 6$

$3 / 6$

$3 / 6$

$3 / 6$

2/6
Each

Lady Clermont, rose-scarlet in tensely blotched with black 2/6

Lady Clementine Mitford,

peach colour, deeper margin ; very beautiful

Lady Clementine Walsh. pinkish white, brown centre

Lady Eleanor Cathcart, rose with chocolate spots

Lady Falmouth, clear rose with intense black blotch

Lady Francis Crossley, rosepink 2/6

Lady Grey Egerton, French

grey with faint blush ; extra fine

Lady Rolle (Veitch), white changing to blush, upper petal brownish yellow

Lady Tankerville, pale rose, white centre

Limbatum, white margined with crimson

Lord Eversley, very dark scarletcrimson with black spots

Lord Roberts, rosy scarlet, black spots ; late

Madame Miolan Carvalho, white with yellow blotch, fine truss 2/6 \&

Madame Wagner, bright pink, white centre

Marchioness of Lansdowne, pale rose with black spots; most striking and distinct

Marie Stuart, blush with maroon spot

Marie Van Houtte, white, slightly spotted

Martin Hope Sutton, scarlet, richly marked; very showy $3 \mathbf{6}$

Maximum triumphans, bright scarlet $\mathbf{3} / \mathbf{6}$

Maxwell T. Masters, rose-crimson ; fine truss and foliage

Melton, rich purple, darker centre 3/6

Memoir, white; one of the best 5/-

Meteor, fiery crimson $\quad$ 2/6

MichaeI Waterer, bright scarlet, good foliage

Minnie, blush-white, large blotch of chocolate spots

Mirabile, deep vivid rose, large

2/6

Miss JekyII, cream-white with chocolate blotch

Mont Blanc, white, dwarf habit 


\section{GENERAL COLLECTION-continued.}

Mrs. Anthony Waterer, white; very fine

Mrs. Arthur Hunnewell, pink with primrose centre

Each

$2 / 6$

Mrs. Beresford Melville, white, faintly tinted with pink $\mathbf{3} / \mathbf{6}$

Mrs. Charles Butler, lilac purple 5/-

Mrs. Chas. Sargent, pink, fine truss $3 / 6 \& 5 /=$

Mrs. Charles Thorold, bright pink, yellowish centre

Mrs. E. C. Stirling, very large trusses of blush-pink flowers 5/-

Mrs. Frederick Hankey, salmonred, richly spotted 2/6

Mrs. F. J.Kirchner, cream-white, spotted

Mrs. F. Phillips, deeprose, lighter centre

Mrs. Harry Ingersoll, purple primrose centre

Mrs. Holford, rich salmon-pink, large truss, extra fine $2 / 6 \& 3 / 6$

Mrs. John Clutton, white, one of the best whites in cultivation $2 / 6 \& 3 / 6$

Mrs. John Kelk, clear rose, late Mrs. John Penn, bright salmonpink, fringed edge

Mrs. John Walter, white centre with bright crimson edge $2 / 6 \&$

Mrs.John Waterer, rose-crimson

Mrs. J. P. Lade, French-grey, deeper centre, very distinct $\mathbf{3 / 6}$

Mrs. Mendel, pink striated with white, yellow centre

Mrs. Milner, rich crimson

Mrs. R. G. Shaw, light blush, with dark maroon blotch ; large truss, distinct

Mrs. Russell Sturgis, white with chocolate spots

Mrs. Shuttleworth, scarlet, lighter centre, much spotted

Mrs. S. Simpson, white beautifully spotted $\mathbf{2} / \mathbf{6} \& \mathbf{3} / \mathbf{6}$

Mrs. Tom Agnew, pure white, with lemon blotch

Mrs. William Agnew, pale rose, lighter edge, yellow centre 2/6 \&

Mrs. William Bovill, rich rosescarlet, very handsome

Neilsonii, brilliant rose-lake, fine truss and free bloorner

* Nobleanum, scarlet and rose 2/6 \&

* - coccineum, fine bright scarlet

* These are dwarf free-flowering varieties, and very early bloomers
Ochroleucum (Veitch), pale yellow with brown spots, very dwarf habit

Old Port, rich plum colour, distinct Othello, crimson-mauve

Pelopidas, crimson

Picturatum, cream-white, chocolate blotch

Pink Pearl, one of the finest Rhododendrons in cultivation $5 /-$ to $21 /$ -

Prince Camille de Rohan, French-white, shaded with rose 2/6

Princess Mary of Cambridge, white centre with rose-purple edging

Promethéus, dark scarlet, black spots ; large truss

Purity, white with a faint yellow eye, very distinct

Ralph Sanders, purplish-crimson; very fine truss

R. S. Field, scarlet, large truss

Sappho, white, blotched maroon

Scipio, rose with deep red $2 / 6$ \& $2 / 6 \& 3 / 6$

Sefton, maroon-crimson

Sigismund Rucker, magentacrimson, finely spotted

Silvio, rich purple, yellow centre

Sir Arthur Guinness, clear rose, very large truss

Sir Humphrey de Trafford, bright rose, yellow centre

Sir Thomas Sebright, rich purple, distinct bronze blotch 2/6

Snowflake, pure white $\quad 2 / 6 \& \quad 3 / 6$

Stella, pale rose with intense

chocolate blotch on upper petal 2/6

Strategist, very bright rosy-pink $3 / 6$

St. Simon, purplish crimson, spotted 2/6

Sylph, bright rose pink $\quad 3 / 6$

The Countess, blush, changing to white

The Crown Prince, bright rose with yellow blotch $\mathbf{2 / 6}$

The Queen, blush changing to white

$2 / 6$

The Warrior, rose-scarlet, large flower

Titian, bright rose-scarlet $\quad 2 / 6$

Vandyke, rose-crimson 2/6

Vauban, mauve, rich yellow blotch $2 / 6$

Vesuvius, deep crimson-scarlet $\quad 2 / 6$

Vivian Grey, rose-pink, spotted 2/6

William Ewart Gladstone, rich, rose-crimson, immense truss 


\section{RHODODENDRON CATAWBIENSE HYBRIDS.}

See General List for Description.

Album elegans Alexander Dancer Caractacus Charles Dickens Charles S. Sargent delicatissimum Everestianum fatuosum fl. pl. giganteum

\section{Guido}

Henrietta Sargent

H. H. Hunnewell

H. W. Sargent

James Bateman
Kettledrum

King of the Purples

Lady Armstrong

Mrs. Milner

purpureum elegans

- grandiflorum

roseum elegans

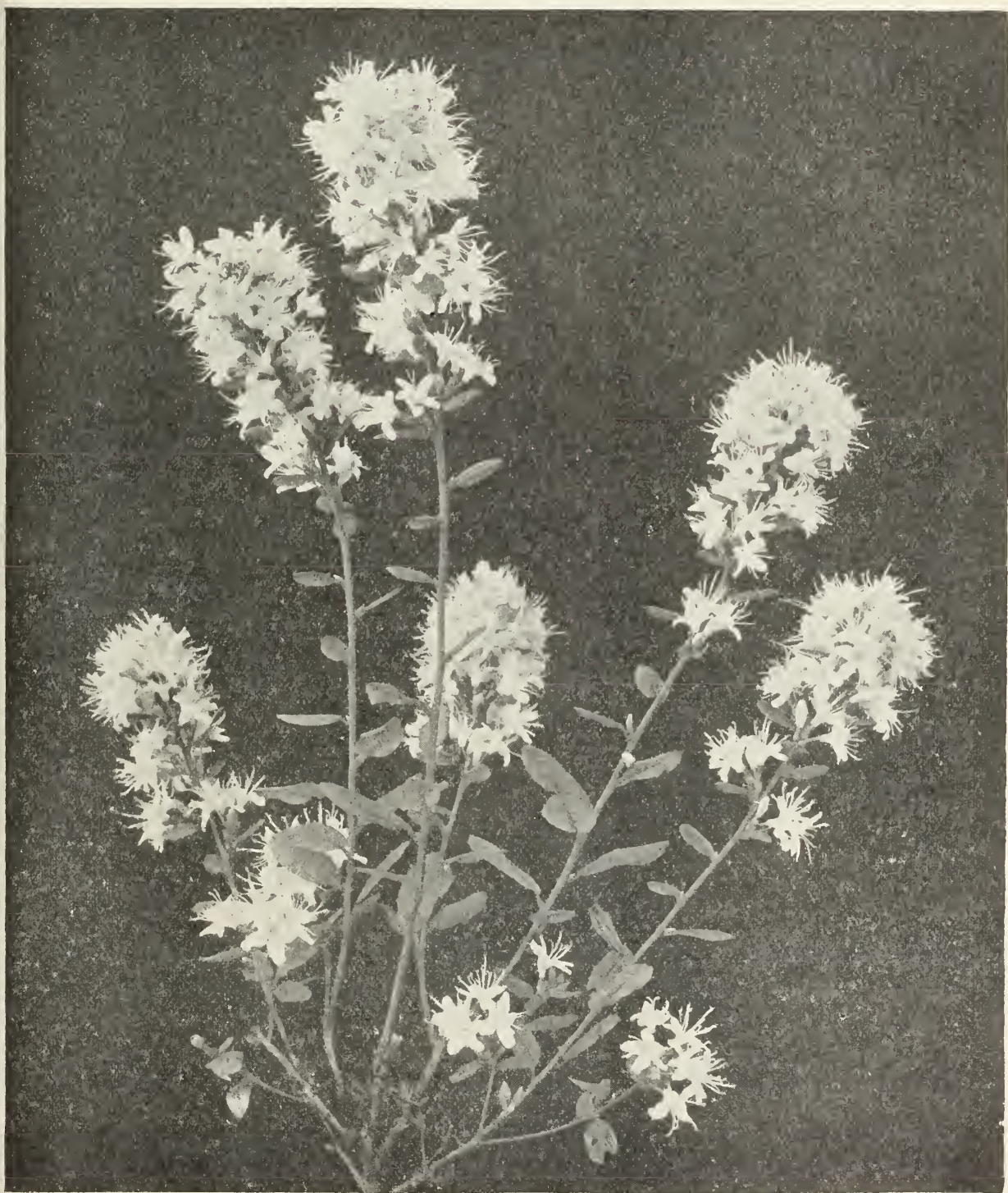

Rhododendron racemosum (see page 22). 


\section{EARLY-FLOWERING HYBRID RHODODENDRONS.}

\section{See General List for Description.}

Altaclarense
Arboreum Wellsianum
Auguste van Geert
Baron Osy
Blanche Superbe
Brilliant

Altaclarense

Auguste van Geert

Blanche Superbe

Brilliant

Broughtonianum
Caucasicum album
- pictum
Limbatum
Madame Wagner
Mirabile

\author{
Nobleanum \\ - coccineum \\ Ochroleucum \\ Prince Camille \\ de Rohan
}

Vesuvius

\section{RHODODENDRON SPECIES AND THEIR VARIETIES.}

Each
2/6
2/6
2/6

- pictum, pale rose

concinnum, dwarf, densely

branched habit, flowers produced

in clusters, pale yellow faintly

spotted on the upper petals, per-

fectly hardy (see illustration, $p .23$ ) 5/-

ferrugineum, rose-pink, a dwarf alpine species

- album, white

fragrans, pale rose

$1 / 6 \& 2 / 6$

hirsutum, light rose-pink $\quad 1 / 6$

- album, white

$2 / 6$

indicum, Krempferi, large,

single, dull brick-red flowers $\mathbf{3 / 6}$

intricatum (see illustration, p. 18) 3/6

\section{DWARF HYBRID RHODODENDRONS.}

Useful for edgings to American beds, for bedding and for Rockwork.

ponticum, rosy-purple (see below).

racemosum, quite hardy, of dwarf and compact habit, flowers about an inch in diameter, produced in dense clusters; white with a soft flush of rose-pink, passing into bright rose at the tips of the segments (see illustration, $p .21$ )

$2 / 6$ to $5 /=$

Smirnowii, rose

sutchuenense, very fine species, bright rose-pink flowers, 3 inches in diameter, borne in dense trusses during March. Foliage large, thick and leathery. Of the large flowered Chinese species collected by E. H. Wilson, this is the first to blossom in cultivation ; does best in partial shade, 10/6
Each

arbutifolium, rose

Early Gem, rosy-lilac, dwarf and

free flowering; highly recom-

mended for pots for winter decor-

ation

$1 / 6 \& 2 / 6$

$1 / 6$

\section{RHODODENDRON PONTICUM.}

One of the most useful for general planting. Good well-rooted vigorous plants, suitable for planting in woods and covers at the following reasonable prices:

Good Plants $30 /-$ to $84 /-$ per 100 ; Larger $12 /-$ to $42 /-$ per dozen. Rhododendron ponticum variegatum

$1 / 6$ to $2 / 6$ each.

\section{RHODODENDRON PONTICUM AND CATAWBIENSE HYBRIDS.}




\section{HIMALAYAN RHODODENDRONS.}

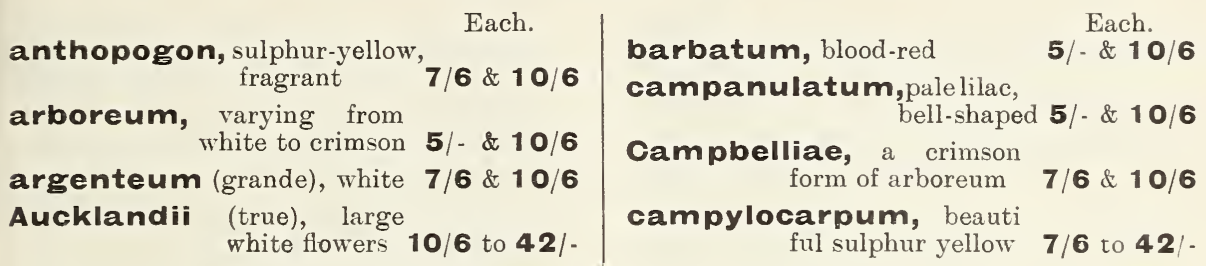

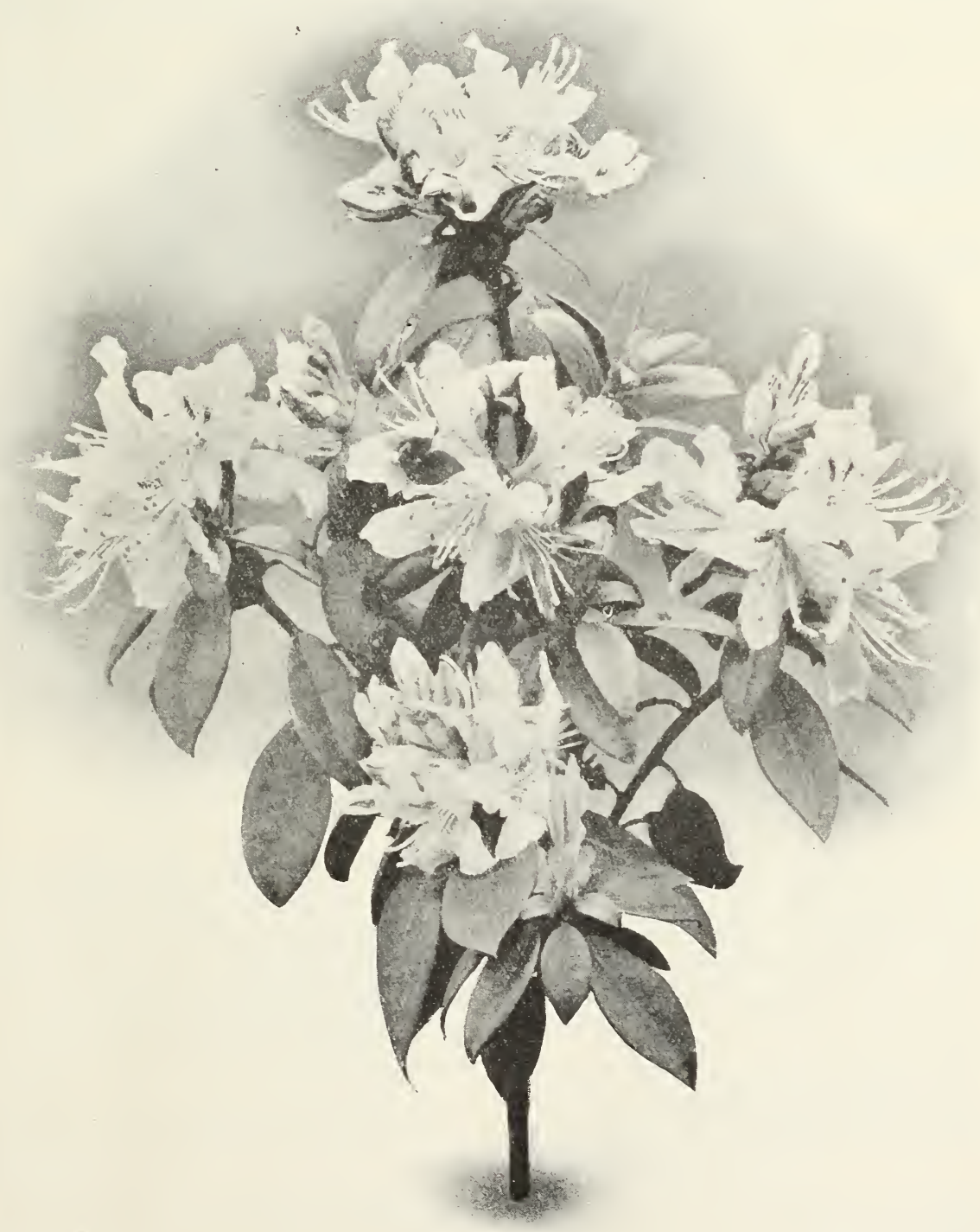

Rhododendron concinnum (see page 22). 


\section{HIMALAYAN RHODODENDRONS (continued)}

Each.

cinnabarinum, orange-red $5 / \cdot$ \& $10 / 6$

- Roylei, blood-red

$7 / 6 \& 10 / 6$

cinnamomeum, white

spotted with purple 10/6

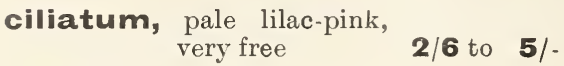

decorum, white, tinged

pink, fragrant $\mathbf{7} / \mathbf{6} \& \mathbf{1 0 / 6}$

Falconeri, dense globose heads of cream coloured flowers $\mathbf{7} / \mathbf{6} \& \mathbf{1 0 / 6}$
Fortunei, mauve-pink, fragrant

Each.

fulgens, brilliant scarletcrimson

$\mathbf{7} / \mathbf{6}$ to $\mathbf{2 1 / .}$

glaucum, small pink

$$
\text { flowers } \quad 5 / \text { to } 10 / 6
$$

Hodgsonii, delicate pale rose-purple flowers 15/- \& 21/.

Manglesii, blush, large trusses $\mathbf{7} / \mathbf{6}, \mathbf{1 0} / \mathbf{6} \& \mathbf{2 1 /}$.

niveum, pale lilac, purple spotting

$7 / 6 \& 10 / 6$

Thomsonii, deep blood-red $\mathbf{7} / \mathbf{6} \& \mathbf{2 1 /}$ -

\section{HYBRIDS.}

Duchess of York, soft salmon pink, upper petals spotted green

$5 /$. to $10 / 6$

Kewensis, white, suffused with pale rose

Each. $7 / 6 \& 10 / 6$

Mrs. Butler, lilac-purple, fragrant flowers $5 /=\& 10 / 6$

Duke of York, bright rosy pink, brown spots on upper petals, free flowering

Mrs. Thiselton Dyer, rose-pink, rich maroon blotch, very large flowers $\mathbf{5 / .} \& \quad \mathbf{7} / \mathbf{6}$ For other Himalayan Varieties see Catalogue of Indoor Plants free on Application.

\section{HARDY AZALEAS.}

\section{PONTICA AND GHENT AZALEAS.}

The finest English and Continental varieties, strong plants well set with bloom buds.

\section{OUR SELECTION.}

Finest named varieties, good blooming plants 24s., 30s., 42s. and 60s. per doz.

Finest varieties, unnamed, mixed 18s. and $24 s$. 12s. and $18 \mathrm{~s}$.

Common, sweet-scented, yellow selection suitable for forcing ... 24s., 30s. and 42 s.

Admiral de Ruyter, blood-red

Altaclarensis, deep orange

Astreans, fine orange

Auguste Mechylinck, white

Aurore de Royghem, yellow shaded with orange

Beaute Celeste, bright rose

Belle Merveille, light pink

Bijou des Amateurs, magenta-rose

Bouquet de flore, salmon-pink ; centre of lobes striped with white

Bronze Unique, blood-red

Charlemagne, orange

Cardinal, bright pink spotted with
Coccinea speciosa, orange-red

Comte de Flanders, carmine

Cymodoce, crimson

Daviesii, white

Domenico Scassi, salmon-pink

Duc de Provence, vermilion-red

Eugénie, blood-red

Fama, mauve

Flameola incarnata, bright pink, yellow spots

Fritz Quihou, deep red throughout

Furst Camille von Rohan, light

Géant des Batailles, carmine 


\section{PONTICA AND GHENT AZALEAS (continued)}

General Trauff, purplish red Glauca stricta, white

Gloria Mundi, vermilion-red

Graf Alfred von Niepperg, salmonpink

Grand Duc de Luxembourg, dark red

Grand Monarque, salmon-pink

Grandeur Triomphant, amaranth shaded with orange

Guelder Rose, bright orange

Heureuse Surprise, milk-white shaded rose

Ignea Nova, purplish carmine

Josephine Klinger, crimson Joseph N. Baumann, blood-red Julda Schipp, blood-red Julius Caesar, dark crimson

Madame Joseph Baumann, salmon-pink
Madame Gustav Guillemot, bright

Madame Moser, pink striated white Marie Verschaffelt, light rose

Mathilde, blush-white

Melaine, light pink

Meteor, brilliant red

Minerva, salmon-rose

Nancy Waterer, orange

Pallas, magenta-red

Prince Henry des Payes Bas, blood-red

Princess Adrienne, deep blood-red

Pucella, purplish crimson

Queen Victoria, magenta-rose

Rembrandt, crimson, shaded violet

Sang de Gendbrugge, deep crimson

Unique, golden yellow

Viscocephala, white

William III., deep orange

\section{AZALEA MOLLIS.}

A race of Azaleas of excellent habit, producing good foliage and large trusses of flowers in many shades of colour; the flowers are larger and of more perfect form than those of the Belgian and Pontica varieties. They are perfectly hardy, and are invaluable for forcing.

Named varieties, $30 /-$ to $42 /-$ per dozen; Standards, $7 / 6 \& 10 / 6$ each. Seedlings, $18 /-$ to $30 /-$ per dozen; $100 /-$ to $150 /-$ per 100 .

Admirable, fine rose

Alphonse Lavallée, bright orange Ambroise Verschaffelt, orangèred Baron Constant de Rebecque, deep yellow

Baron Edmund de Rothschild, red Bouquet d'Orange, bright orange

Charles Lupis, rose shaded with magenta Chevalier A. de Realii, straw-yellow Comte de Gomer, bright pink Comtesse de Kerchove, orange-red Comte de Quincey, bright yellow Comte Papadopoli, rose-pink Consul Ceresole, red shaded with pink
Consul Pecher, bright pink

Dr. Léon Vignes, white tinted with yellow Ébenezer Pycke, salmon-pink

Elizabeth, claret

Ernest Bach, salmon-pink shaded with orange

Isabel van Houtte, dark yellow

Madame Caroline L. d'Hanis, pink

M. Arthur de Warelles, light carmine

M. Koster, fine red

Professor M. Koster, deep red

Queen Sophia, bright orange

T. J. Seidel, bright pink

W. E. Gumbleton, golden-yellow 


\section{AZALEA MOLLIS HYBRIDS.}

\section{A. Mollis $\times$ A. Pontica.}

\section{2/6 each.}

Charles Rogier, mauve

Dulcinee, orange-red

Edison, pink

Esmeralda, dark orange

Frederic de Mérode, red

Frère Orban, cream-white

General Brialmont, mauve

General Goffinet, pinkish-mauve

Gloire de Belgique, red, shaded lilac.

Henry Conscience, pinkish-white
Heros de Flandre, bright pink, shaded yellow

Mignon, rose-pink

Mons. Desbois, scarlet-rose

Oswald de Kerchove, pink

Prince Albert, salmon-pink, shaded cream

Prince Baudouin, bright rose

Souvr. de L. van Houtte, white and pink, spotted yellow

\section{A. Mollis $\times$ A. Sinensis.}

$3 / 6$ each, except where otherwise priced. Seedlings, $24 /-$ \& $30 /-$ doz.

Alma Tadema, soft rose-pink

Anthony Koster, rich yellow (see illustration $p .27$ )

Betsy de Bruin, orange, crimson blotch, 5/- each

Captain Carsjens, rose, red margin

Charles Maarschalk, bright orange

Dr. Pasteur, orange-red

Dr. Reitenbach, salmon-red

Ellen Cuthbert, salmon, spotted purple,

$$
\text { 5/- each }
$$

Emil Liebig, salmon-red

Franz van der Bom, orange, spotted purple, 5/- each

Garten-Inspector Ohrt, rose, bronze blotch

General Vetter, orange
Glory of Boskoop, deep yellow, shaded

Hortulanus Witte, orange orange, $\mathbf{5} /$ - each Hugo Koster, salmon-red

J. C. van Tol, deep red, 3/6 each

J. J. de Vink, soft rose

Louis Endz, orange-yellow, 5/- each

L. Späth, salmon-pink, shaded rose, spotted orange, 5/- each

Madame Anthony Koster, apple

blossom

N. Beets, dark orange

Peter Koster, orange

Professor Rontgen, light orange, red blotches, $\mathbf{5} /-$ each

Sebastopol, soft red

\section{AZALEA HYBRIDS WITH DOUBLE FLOWERS.}

\section{2/6 each; Standards $15 /-$ to $31 / 6$ each.}

Aida, rose-pink

Apelles, scarlet

Ariadne, white

Byron, white

Corneille, pale rose

Diogenes, pink shading to cream

Fenelon, salmon-rose, paler edges

Freya, pale mauve

Hora, yellowish-pink

II Tasso, light red

Le Titien, white

Mécene, white

Milton, white and pink
Murillo, purplish-pink

Norma, flesh-pink

Phébé, pale yellow and pink

Phidias, blush-white and yellow

Praxiteles, white and pink

Quentin Metsys, bright pink

Rubens, yellowish-white, shaded pink

Racine, pale pink

Teniers, pale flesh tinted rose

Vandyck, straw-yellow, centre and edges shaded pink

Velasquez, cream-white

Virgil, chrome-yellow 


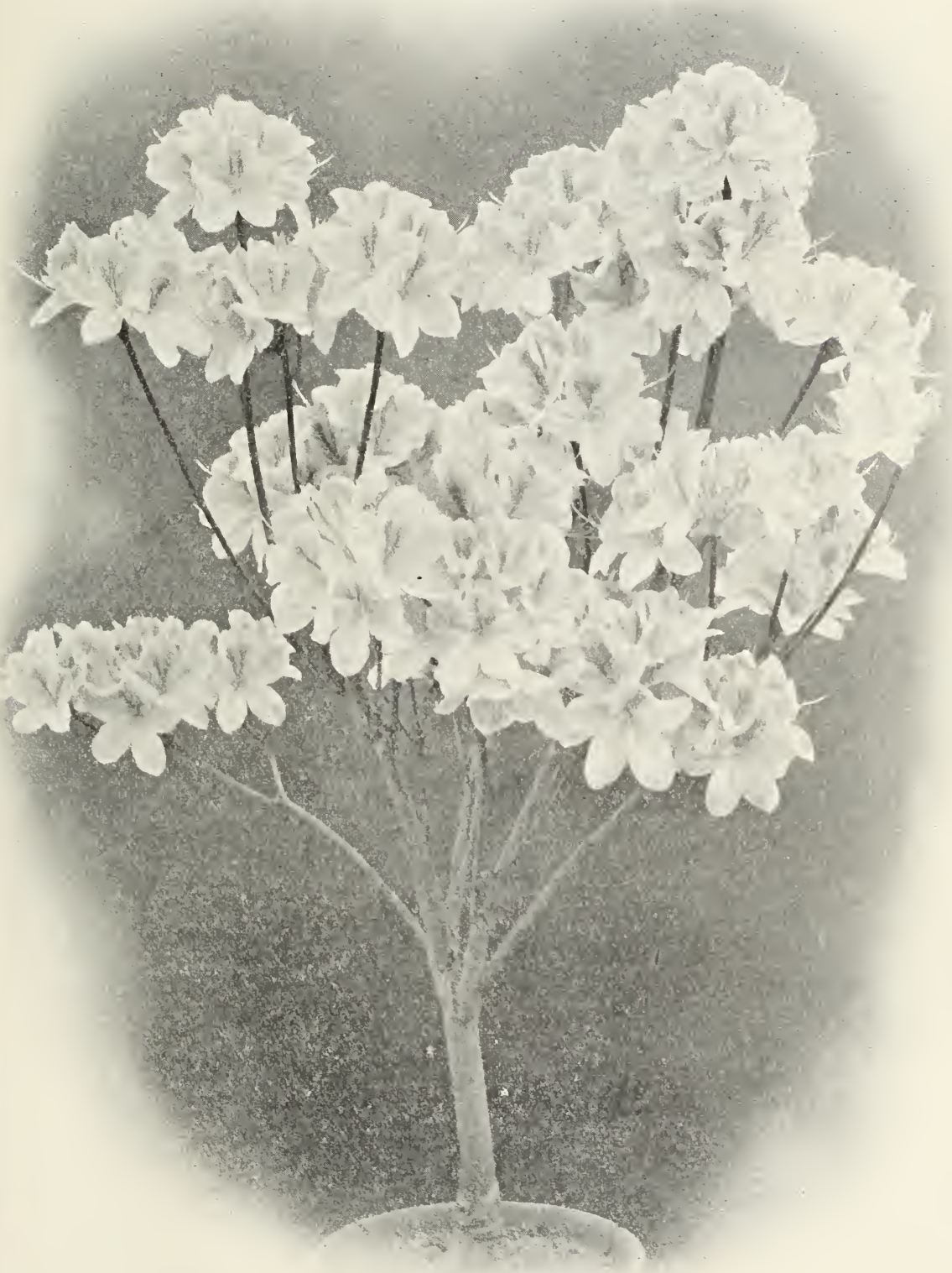

Azalea Anthony Koster.

The best Azalea for forcing and general planting. Colour rich yellow. Blooms during May (see page 26). 


\section{AZALEA PONTICA HYBRIDS WITH DOUBLE FLOWERS.}

\section{$2 / 6$ each; $24 /-$ and $30 /-$ per dozen.}

Bartolo Lazaris, flesh-pink

Bijou de Gendbrugge, white and pink Dr. Streiter, carmine

Graf von Meran, white tinted with pink Heroine, rose-lilac

Leibnitz, egg-yellow bordered with carmine Louise Aime Van Houtte, vermilion and orange
Mina Van Houtte, pink toned with yellow

Narcissifiora, pale yellow

Ophirie, yellow toned with pink

Rosetta, pink

Van Houttei, salmon-pink, spotted golden-yellow

\section{AZALEA SPECIES.}

The following cannot be included in any of the preceding sections. Amcena, obtusa and roscflora are among the best of Azaleas for pot culture. Occidentalis and Vaseyi are handsome American species, Pontica is the common yellow Azalea.

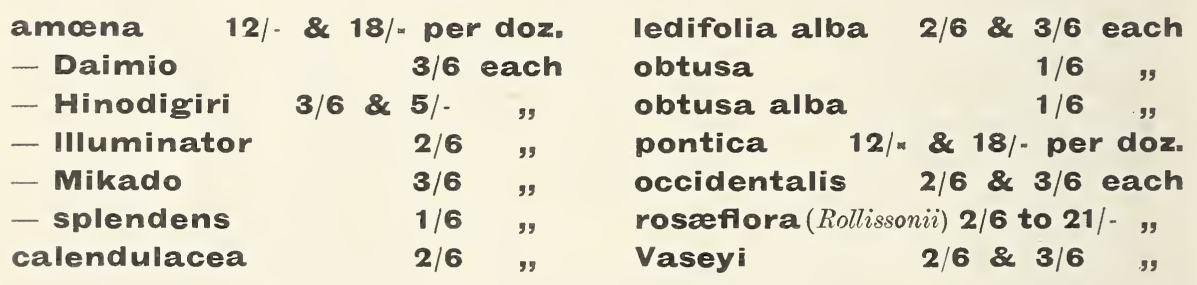

\section{AMERICAN AND JAPANESE PLANTS.}

These require a peat soil, similar to that for Rhododendrons.

Andromeda

arborea. Pure white bell-shaped

flowers, produced in profusion

during Aug. and Sept. $2 / 6$ to

axillaris (Leucothoe)

calyculata (Cassandra)

- minor

Catesbæi (Leucothoe)

floribunda (Pieris) $\quad \mathbf{1 / 6}$ to

formosa (Pieris)

japonica (Pieris)

- albo-marginata

$2 / 6$ to $5 /-$

$3 / 6 \& 5 /-$

Mariana (Pieris)

$2 / 6$

polifolia angustifolia $\quad 1 / 6$

speciosacassinefolia(Zenobia)

$1 / 6 \& 2 / 6$

Bruckenthalia

spiculifolia

$1 / 6$
Bryànthus (Menziesia)

empetriformis

Clèthra

alnifolia

canescens. Numerous milkwhite flowers in panicles upwards of a foot long, produced during the summer months. F.C.C., R.H.S.

Cyrilla

racemiflora. 4 to $5 \mathrm{ft}$., with slenderangular branchesand tufts of dark green leaves 1 to $1 \frac{1}{2}$ inches in length. Many flowered racemes of small pure white flowers, arranged in tufts on the ends of the old wood. A.M., R.H.S. 
AMERICAN AND JAPANESE PLANTS-continued.

\section{Empètrum \\ nigrum \\ - scoticum \\ - tomentosum}

\section{Enkianthus}

campanulatus. Deciduous shrub, abundant Andromedalike dark red flowers

Epigaa

repens

Erica (Hardy Heaths)

$\begin{array}{lr}\text { arborea } & 2 / 6 \text { and } 3 / 6 \\ \text { australis } & 1 / 6 \\ \text { ciliaris } & \\ \text { codonodes (lusitunica) } & 1 / 6 \\ \text { herbacea } \\ \text { - alba } \\ \text { Mackayana } \\ \text { mediterranea } \\ \text { - alba } \\ \text { - hybrida } \\ \text { scoparia } \\ \text { stricta } \\ \text { Tetralix } \\ \text { - alba } \\ \text { - intermedia } \\ \text { - rubra } \\ \text { vagans alba } \\ \text { - carnea } \\ \text { - rubra } \\ \text { Veitchi }\end{array}$

\section{Veitchii} vulgaris alba (Calluna)

- Alportii

- aurea

- cuprea

- fiore pleno

- Foxii

- Hammondii

- monstrosa

- pumila

- rigida

- Searlii

- variegata

Fothergilla alnifolia

Gaultheria

nummularifolia

procumbens

Shallon

- acutifolia

tricophylla

2/6

\section{Each. \\ $1 / 6$ \\ $1 / 6$ \\ $1 / 6$}

\section{Pernettya}

mucronata per dozen

- angustifolia

- speciosa

Philesia

buxifolia

$3 / 6$

$1 / 6$

$1 / 6$

$1 / 6$

Leucothoe

axillaris Catesbai\} See Andromeda p. 29

Davisiae

$1 / 6$

- pumila $1 / 6$

$1 / 6$

$1 / 6$

$1 / 6$ to $5 /-$

$2 / 6 \& 3 / 6$

\section{6}

\section{6}

Menzièsia

empetriformis (Bryanthus) $\quad 1 / 6$

globularis

$2 / 6$

polifolia (Daboecia) (Irish)

- alba

- erecta

- atropurpurea

1/- each

9/- doz.

$50 /-100$

Oxycóccus (Cranberry)

macrocarpus

Oxydendron

arboreum (Andromeda arborea)

Polygala

Chamabuxus purpurea 1/6

Rhodora

canadensis

$1 / 6$

Vaccinium

Myrtillus per dozen 9/- 1/-

ovatum $2 / 6$

pensylvanicum $\quad 2 / 6$

stamineum $\quad 2 / 6$

uliginosum $1 /-$

Vitis-ldaea $1 / 6$

- major $\quad 1 / 6$ 


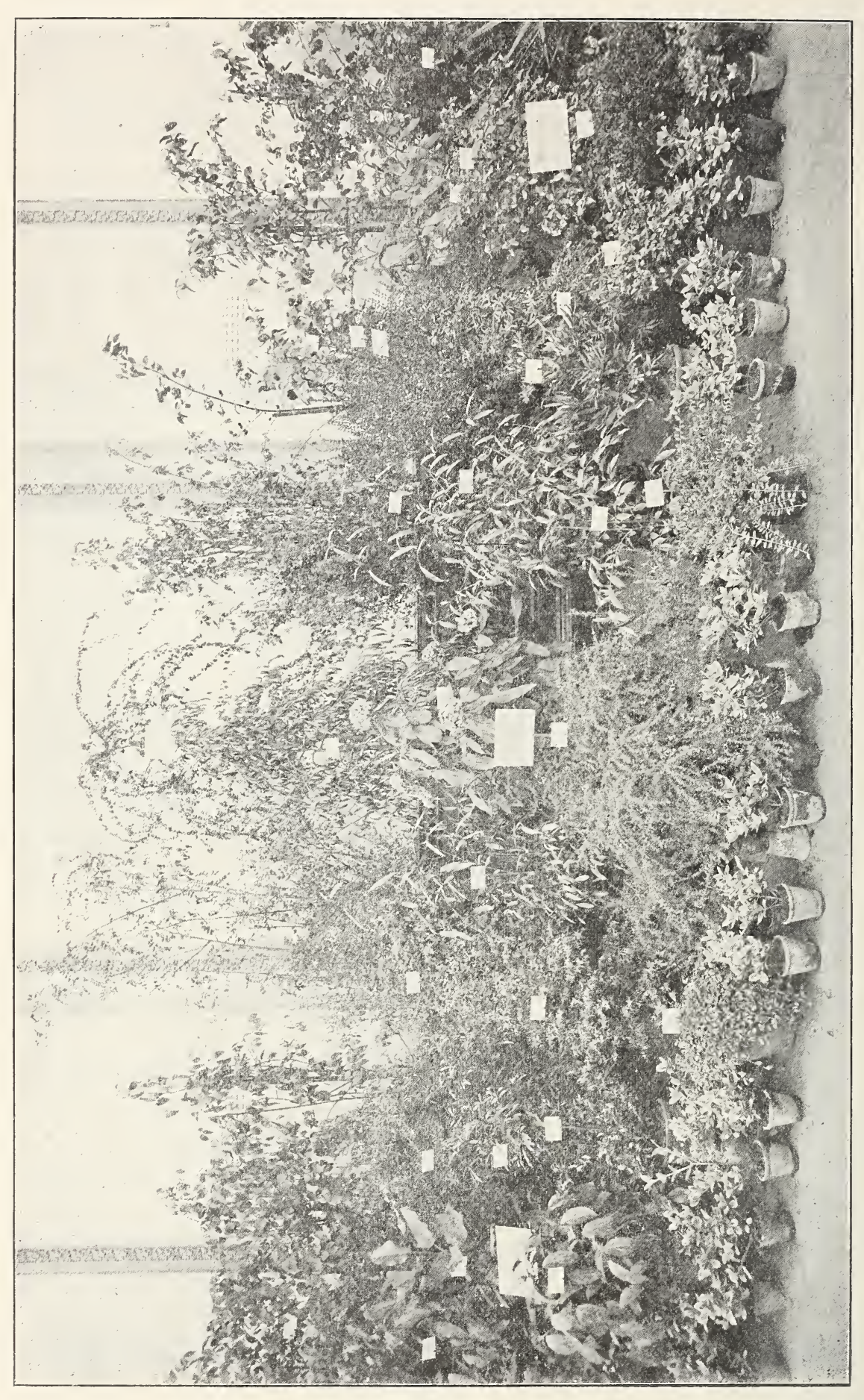

en.

is

$\infty$ :

ร

잉

के के

를 을

ฮซ

$\rightarrow$ of

잉

엉화

눙

응

돈

(1)

응응

50

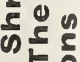

๘

도은

\&

है

के

$\lambda$

한항

똥ํㅇ

404

등용

茾

뚜요

ㅍ

०

สำ

울

55

을

옹옹

4

ำ

오

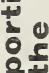

त)

वذ 


\section{NEW AND CHOICE EVERGREEN}

\section{TREES AND SHRUBS.}
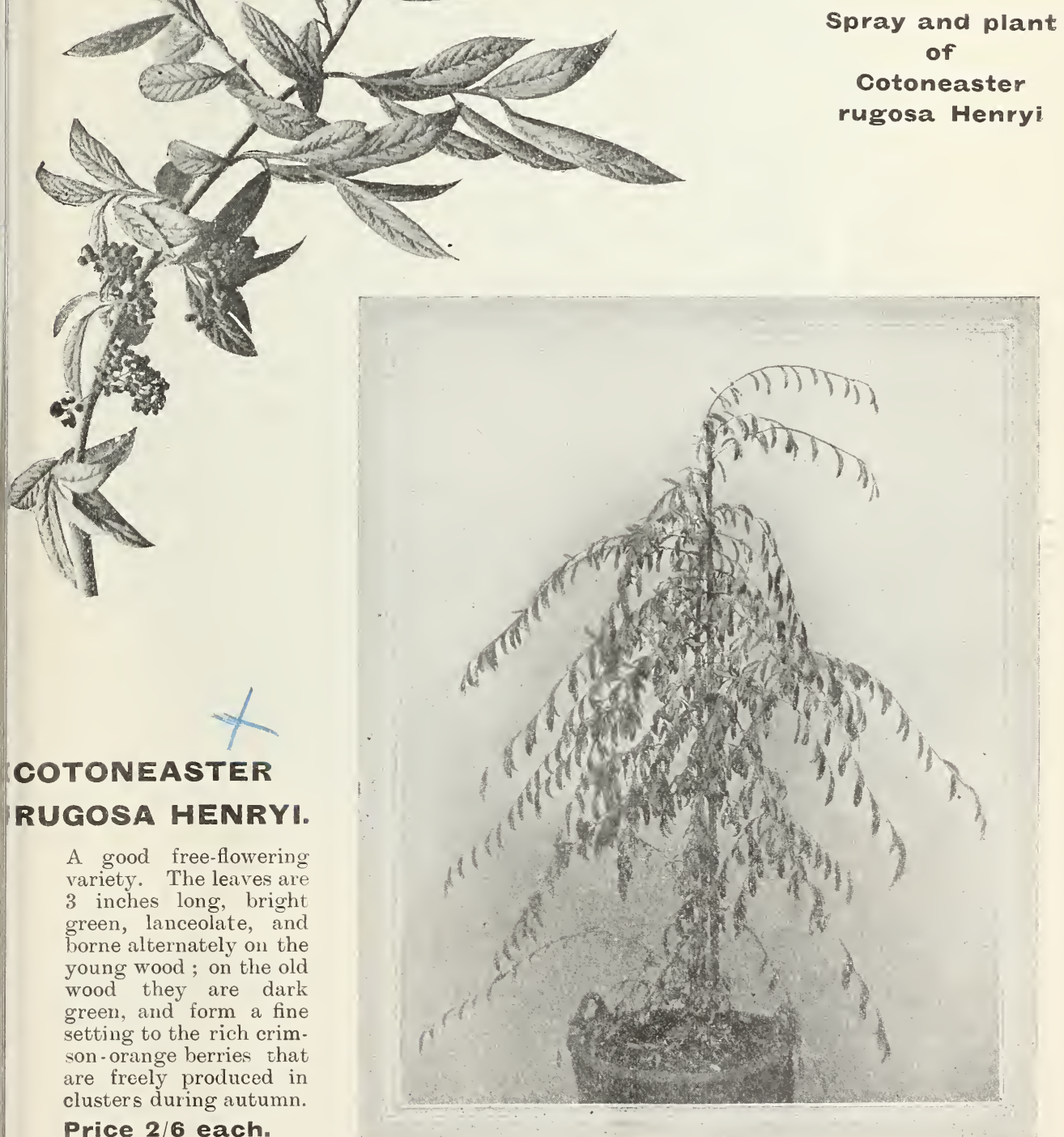

\section{COTONEASTER} RUGOSA HENRYI.

A good free-flowering variety. The leaves are 3 inches long, bright green, lanceolate, and borne alternately on the young wood; on the old wood they are dark green, and form a fine setting to the rich crim. son-orange berries that are freely produced in clusters during autumn. Price 2/6 each. 


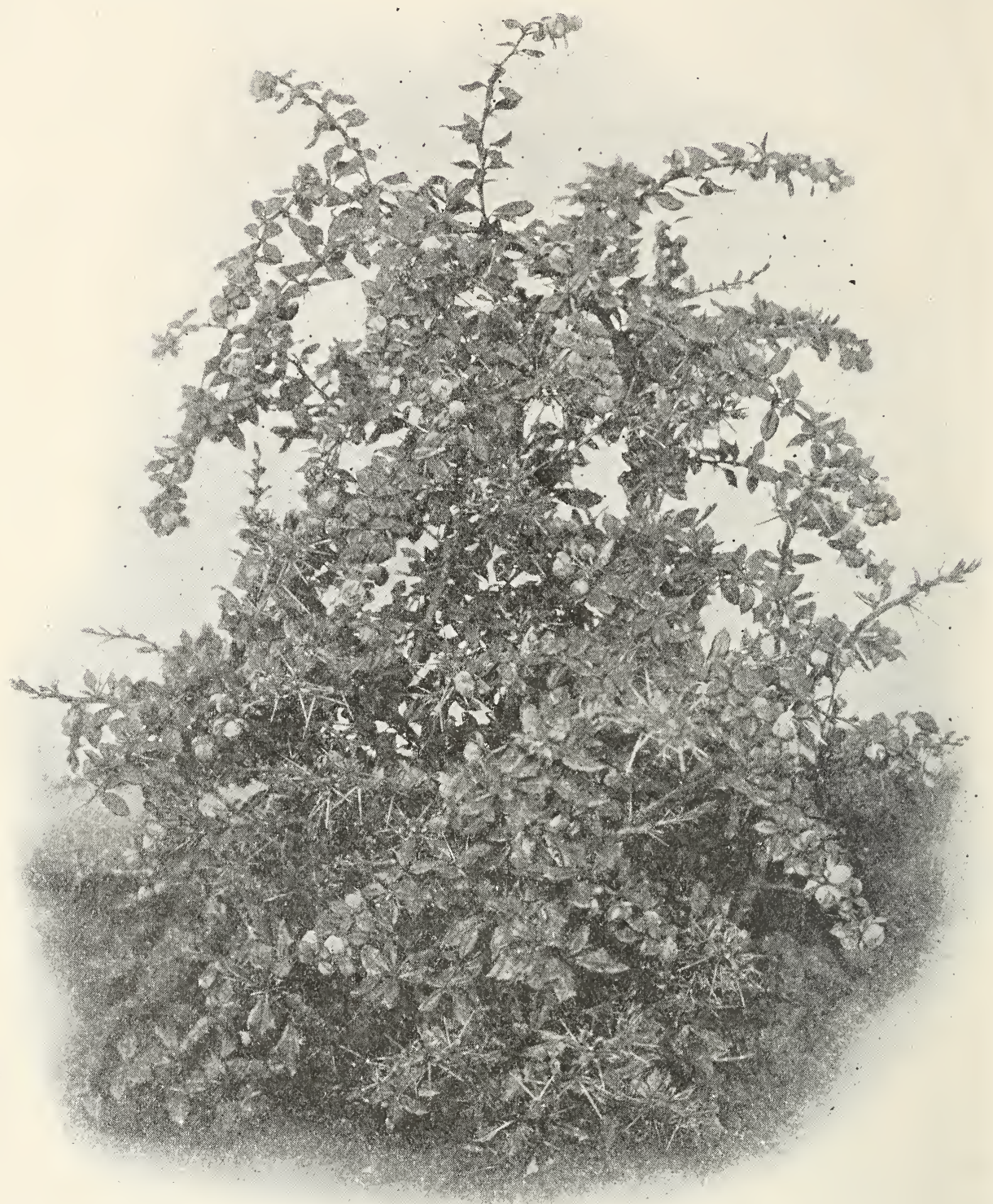

Berberis verruculosa.

\section{BERBERIS VERRUCULOSA}

A dense growing evergreen shrub about 2 feet high. Leaves leathery, ovate, spiny, shining above, glaucous beneath. Flowers yellow, borne in pairs, succeeded by violet purple fruits. A.M. R.H.S. See illustration above.

each $3 / 6$

\section{BERBERIS GAGNEPAINII}

An elegant evergreen Barberry of compact growth flowering freely during June in this country. The pale yellow flowers are succeeded by glaucous purple berries.

Illustrated in Gard. Chron. 1909. Vol. II. p. 226 fig. 96.

each $3 / 6$ 


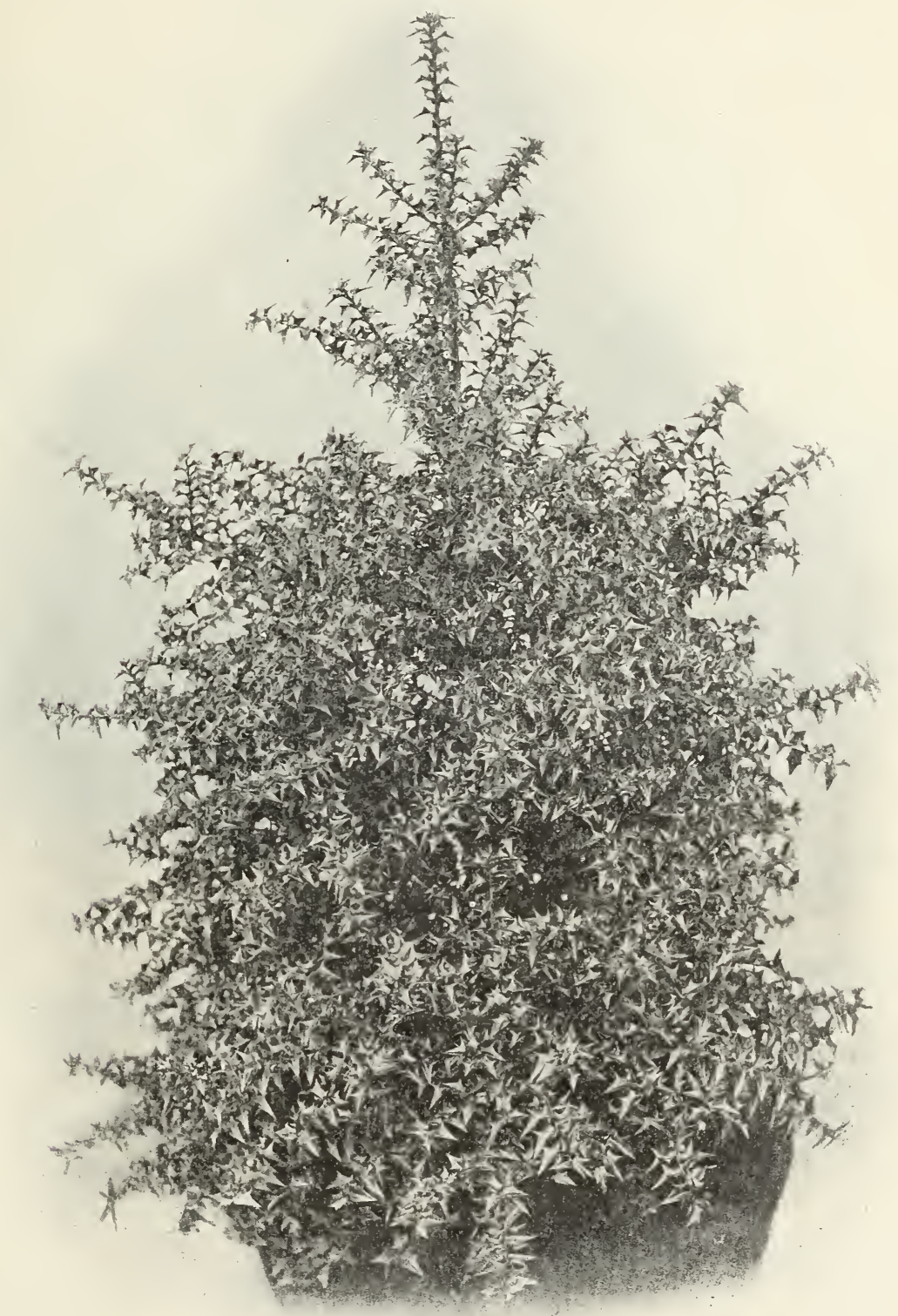

\section{ILEX PERNYI.}

A dense growing very distinct Holly, with leaves about $1 \frac{1}{2}$ inches long, which, on the young wood, are pale green, changing to very dark green on the older shoots. A beautiful neat evergreen of very compact habit (see illustration above).

First Class Certificate from the Royal Horticultural Society. 


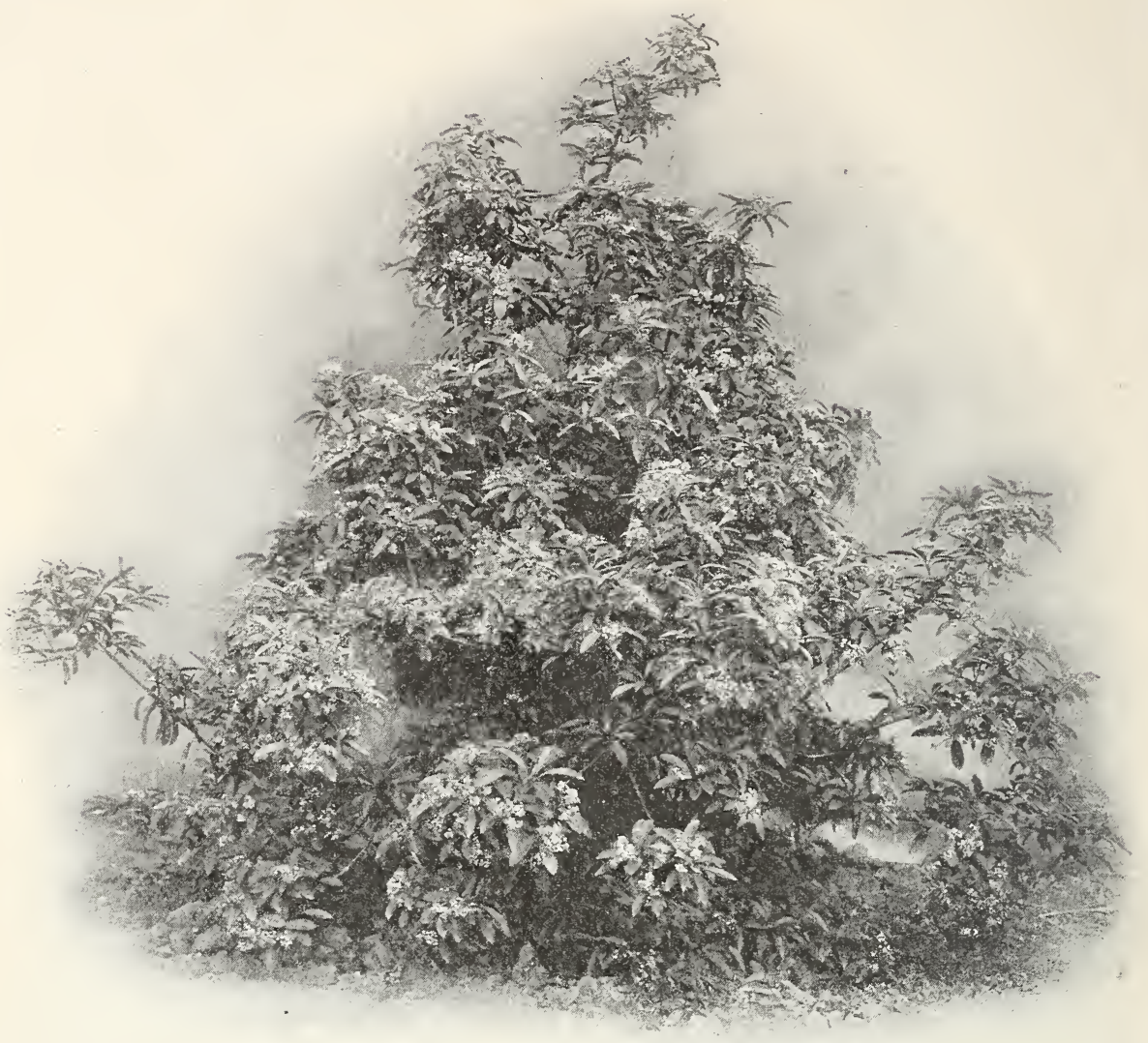

Stranvæsia undulata.

\section{LONICERA NITIDA}

An evergreen shrub of very neat habit 3 to $6 \mathrm{ft}$. high. Branches erect growing, densely covered with small ovate leathery leaves that have a brilliant gloss on the upper surface. Fragrant cream-white flowers. A most distinct Bush Honeysuckle $5 /-\mathbf{e a c h}$

\section{LONICERA PILEATA.}

A dwarf spreading honeysuckie with very small neat leaves of pale green colour. A most desirable subject for the rock garden.

$1 / 6$ each

Award of Merit from the Royal Horticultural Society.

Fig. in Bot. Mag. T. 8060 .

\section{STRANVAESIA UNDULATA}

A handsome evergreen plant resembling a Photinia, which, on account of its orange red fruits produced in corymbs, is a valuable acquisition for the shrubbery.

See illustration above.

$3 / 6$ each

\section{VIBURNUM RHYTIDOPHYLLUM.}

A very striking evergreen shrub with remarkable foliage. Its broadly lanceolate leaves are from 8 to 9 inches in length and 2 to $2 \frac{1}{2}$ inches broad. The upper surface, being dark green and much channelled, forms an agreeable contrast to the underside, which is covered with a dense dun-coloured tomentum. The vigorous growths are terminated by corymbs of yellowish-white flowers, standing well above the foliage, giving place in September to dark red berries. Although grown in an extremely cold and bleak position in our Coombe Wood Nursery it has proved quite hardy, retaining its ornamental appearance throughout the Winter months (see illustration on cover). $3 / 6$ to $21 /$ each

First Class Certificate from the Royal Horticultural Society. 


\section{EVERGREEN TREES \& SHRUBS.}

\section{GENERAL COLLECTION.}

Alatèrnus. See Rhamnus p. 39.

Price per plant. Arbutus

Unedo (common)

1/6 to $3 / 6$

- Croomii (scarlet)

- $2 / 6$

- microphylla

- $2 / 6$

- Rollissonii

Arctostaphylos

Uva-ursi

- californica

Aristotelia

Macqui

- variegata

Arundinaria (ste page 43).

Aucuba

Female varieties.

japonica limbata

- fructu-albo

$1 / 6 \quad 2 / 6$

- Iongifolia

$1 / 6 \quad 2 / 6$

$1 / 6 \quad 86$

- maculata (the old A. japonica of gardens)

- vera nana (green)

- viridis

Male varieties.

japonica maculata

- viridis

Azara

dentata

Gilliesii

integrifolia

- variegata

Ianceolata

microphylla

serrata

Bàccharis

patagonica

Bambusa (see page 43)

Benthàmia (Cornus)

fragifera

Berberidópsis

corallina

Bèrberis

acuminata, gracefully arching branches, young wood bright red; leaves sessile, lanceolate and spiny along the margin, bronzy yellow flowers borne in clusters on slender stalks in the axils of the leaves

$2 / 6 \quad 5 /-$
Berberis (continued) Price per plant.

Aquifolium (Mahonia) 6d. 1/6

- fascicularis _ $\quad 1 / 6$

- rotundifolia Hervei - $\quad 1 / 6$

- undulata nana _ $1 / 6$

buxifolia (dulcis) $\quad 9 d, \quad 1 / 6$

- nana - $1 / 6$

congestiflora

hakeoides - $3 / 6$

Darwinii 1/- $2 / 6$

Fortunei $\quad-\quad 2 / 6$

Fremontii $\quad-\quad 3 / 6$

Gagnepainii (see $p .32$ ) - $\quad 3 / 6$

glumacea $\quad-\quad 1 / 6$

ilicifolia — $\quad 1 / 6$

japonica $\quad-\quad 1 / 6$

Knightii $\quad-\quad 2 / 6$

Neubertii $\quad-\quad 1 / 6$

pruinosa $\quad-\quad 2 / 6$

stenophylla (hybrid) 1/- $1 / 6$

trifoliata $\quad-\quad 3 / 6$

verruculosa (see p. 32) - $3 / 6$

Wallichiana (Hookeri) _ $1 / 6$

B. Darvinii and B. Aquifolium make excellent cover for game, for which purpose suitable plants can be supplied by the 100 or 1,000 , at a moderate price.

For Deciduous Species see page 57.

\section{Bupleurum}

fruticosum

$1 / 6$

Buxus (Box Tree)

balearica (Minorca Box) 1/6 2/6 japonica aurea $\quad 1 / 6 \quad 2 / 6$

sempervirens

- argentea

- Handsworthii

- rosmarinifolia

6d. $2 / 6$

- suffruticosa

and upwards

- thymifolia

- 1/6

- $6 d$.

$1 / 62 / 6 \quad$ - thymifolia 6 d. $2 / 6$

\section{Camellia}

Sasanqua $\quad 3 / 6 \quad 5 /=$

Very free flowering. Well adapted for training against a wall. Three varieties, single red, double white, and double pink flowers. F.C.C.R.H.S.

Carpentaria

californica $\quad 3 / 6$ to $5 /=$

2/6 Ceanòthus (see page 87) 


\section{GENERAL COLLECTION-continued.}

Price per plant.

\begin{tabular}{|c|c|c|}
\hline ferasus (Prunus) (Che & & \\
\hline ilicifolia (evergreen Plu & ) - & \\
\hline $\begin{array}{l}\text { Laurocerasus } \\
\text { (common Laurel) }\end{array}$ & 6d. & to \\
\hline - caucasica & $1 /-$ & \\
\hline - colchica & 6d. & \\
\hline - latifolia & $1 / 6$ & \\
\hline - rotundifolia & $1 / 6$ & \\
\hline $\begin{array}{r}\text { - Schipkaensis } \\
\text { (Zabeliana) }\end{array}$ & - & \\
\hline $\begin{array}{c}\text { Iusitanica (Portugal } \\
\text { Laurel) }\end{array}$ & $9 d$. & \\
\hline - azorica & $1 / 6$ & \\
\hline - myrtifolia & $1 / 6$ & \\
\hline
\end{tabular}

Standard Portugal Laurels, with excellent heads and clean straight stems, both of the common form and of the variety myrtifolia, can be supplied at prices varying to the size of the plants.

\section{Choisya}

ternata

$1 / 6 \quad 3 / 6$

\section{Cistus}

angustifolius

cymosus

cyprius

Florentinus

formosus

Each. Perdoz.

$1 / 6 \quad 12 /$

$1 / 6 \quad 12 /=$

$1 / 6 \quad 12 /=$

$1 / 6 \quad 12 /=$

Iadaniferus (Gum Cistus) 1/6 12/=

laurifolius

$1 / 6 \quad 12 /-$

Iusitanicus

$1 / 6 \quad 12 /=$

salvifolius

$1 / 6 \quad 12 /=$

undulatus

$1 / 6 \quad 12 /=$

\section{citrus}

trifoliata (Agle sepiaria) -

2/6

cleyèra

japonica

2/6 to $3 / 6$

- variegata

- $5 /=$

Colletia

cruciata (bictonensis) - 2/6

spinosa (horrida, ferox) - 2/6

\section{Coròkea}

Cotoneaster

Cotoneàster

angustifolia

buxifolia (Wheeleri)

congesta (glacialis)

Franchetii
Cotoneàster (continued)

Price per plant.

horizontalis

$1 / 6$ to $2 / 6$

humifusa, a creeping

species of dense growth,

long trailing shoots covered

with small dark green

leaves, admirably adapted

for planting on steep

banks and in the niches

of a rockery ; scarlet

fruits in autumn

- $1 / 6$

microphylla

6d. to $1 / 6$

pannosa

$1 / 6$

rugosa Henryi (see page 31)

rupestris

thymifolia

uniflora

$\begin{array}{ll}- & 1 /- \\ - & 1 /- \\ - & 1 /=\end{array}$

See also Deciduous List, page 61.

\section{Cratagus}

Pyracantha (Evergreen

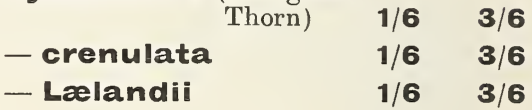

\section{Dàphne}

Blagayana $\quad-\quad 3 / 6$

Cneorum $\quad-\quad 1 / 6$

hybrida (Dauphinii) $\quad \mathbf{1 / 6} \quad 2 / 6$

Laureola (Spurge Laurel) 6d. $\quad 1 / 6$

- purpurea _ 3/6

neapolitana (Fioniana) $1 / 6 \quad 2 / 6$

pontica $\quad-\quad 1 / 6$

\section{Daphniphyllum}

glaucescens

$$
\text { (macropodum) 2/6 5/- }
$$

Leaves ovate-lanceolate, 6 to 8 inches long, dark glaucous green on the upper surface, paler and more glaucous beneath with reddish petiole and midrib. A distinct specimen for the lawn or border.

- viridis (concolor) $\quad 2 / 6 \quad 5 /=$

Desfontainea $\begin{array}{lll}\text { spinosa } & 2 / 6 & 3 / 6\end{array}$

Diplopáppus (Cassinia) chrysophyllus (fulvida) - $1 / 6$ leptophyllus _ _ $\quad 1 / 6$

Drimys

Winteri (Winter's Bark) $1 / 6 \quad$ 2/6 


\section{GENERAL COLLECTION-continued.}

\begin{tabular}{|c|c|c|}
\hline glabra & - & $1 / 6$ \\
\hline - folio-variegata & - & $1 / 6$ \\
\hline macrophylla & $1 / 6 \mathrm{t}$ & $2 / 6$ \\
\hline pungens & $1 / 6$ & $2 / 6$ \\
\hline - aureo-maculata & $1 / 6$ & $2 / 6$ \\
\hline - - picta & $1 / 6$ & $2 / 6$ \\
\hline - variegata & $1 / 6$ & $2 / 6$ \\
\hline
\end{tabular}

See also Deciduous List, page 64.

$\begin{array}{lrr}\begin{array}{l}\text { Embòthrium } \\ \text { coccineum }\end{array} & 5 /- & 10 / 6 \\ \begin{array}{l}\text { Escallonia } \\ \text { exoniensis (hybrid) }\end{array} & - & 1 / 6 \\ \text { illinita } & 1 / 6 & 2 / 6 \\ \text { langleyensis (hybrid) } & - & 2 / 6\end{array}$

Bright rose-carmine flowers, dark lustrous green leaves. A.M.R.H.S.

\begin{tabular}{lll} 
macrantha & $1 /-$ & $2 / 6$ \\
- Ingramii & $1 / 6$ & - \\
- sanguinea & $1 / 6$ & - \\
montevidensis & & \\
\multicolumn{1}{c}{$\quad$ (floribunda) } & $1 / 6$ & - \\
Philippiana & $2 / 6$ & - \\
pterocladon & - & $1 / 6$ \\
rubra & - & $1 / 6$
\end{tabular}

\section{Eucalyptus} coccifera $\quad 1 / 6$ to $2 / 6$ Gunnii

Eugènia (Myrtus)

$\begin{array}{lll}\text { apiculata } & 1 / 6 & 2 / 6 \\ \text { Ugni } & 1 / 6 & 2 / 6 \\ \text { - variegata } & 1 / 6 & 2 / 6\end{array}$

\section{Euónymus}

fimbriatus (pendulus) $\quad-\quad 1 / 6$

japonicus 9 d. $2 / 6$

- aureo-marginatus

(ovata)

$1 / 6 \quad 3 / 6$

- argenteo-

variegatus $1 / 6 \quad 2 / 6$

- aureo-variegatus

(picta)

$1 / 6 \quad 2 / 6$

- latifolius albo-

variegatus $1 / 6 \quad 2 / 6$

- microphyllus
Euonymus

Price per plant. radicans variegatus $9 \mathrm{~d}$. to $1 / 6$ - Silver Gem

$1 / 6 \quad 2 / 6$

E. radicans variegatus is a hardy, dwarf, prostrate-growing variety, well adapted for edgings or filling beds where a permanent bright silver-edged plant is required. We can supply this useful plant by the 100 or 1,000 at very moderate prices.

\section{Eurya}

japonica (Fortunei) $\quad \mathbf{1 / 6}$ to $2 / 6$

$\begin{array}{lll}- \text { angustifolia } & 1 / 6 & 2 / 6\end{array}$

- latifolia variegata $1 / 6 \quad 3 / 6$

Eurybia. See Olearia p. 38.

Fàbiana

imbricata $\quad-\quad 2 / 6$

Gàrrya

elliptica

- fomina

$1 / 6$ to $2 / 6$

Thuretii

$1 / 6 \quad 2 / 6$

Grevillea

$\begin{array}{lll}\text { rosmarinifolia } & 1 / 6 & 2 / 6 \\ \text { sulphurea } & 1 / 6 & 2 / 6\end{array}$

Griselinia

littoralis $\quad \mathbf{1 / 6}$ to $2 / 6$

- macrophylla $\quad$ - $3 / 6$

\section{Hedera}

arborea (Tree Ivy) $\quad 1 / 6 \quad 2 / 6$

$\begin{array}{lll}\text { - chrysophylla } & 1 / 6 & 2 / 6\end{array}$

$\begin{array}{lll}\text { - elegantissima } & 1 / 6 & 2 / 6\end{array}$

Standards of these Tree Ivies can be supplied. Particulars on application.

Helianthemum (Rock Cistus)

in variety

9d.

6/- per doz.

Hymenanthèra

crassifolia

Hypericum

calycinum, 4/- doz. ;

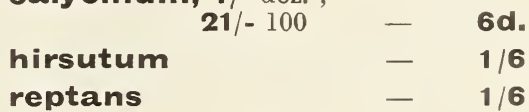

Ilex (Holly)

Aquifolium (common Holly), fine bushy plants, from 1 to

5 and 6 feet

6d. to $15 /=$

- Broad silver

2/6 42/-

- flavescens (yellow-

berried) $26 \quad 5 /$

Golden King $3 / 6 \quad 5 /=$

- Queen 2/6 42/-




\section{GENERAL COLLECTION-continued.}

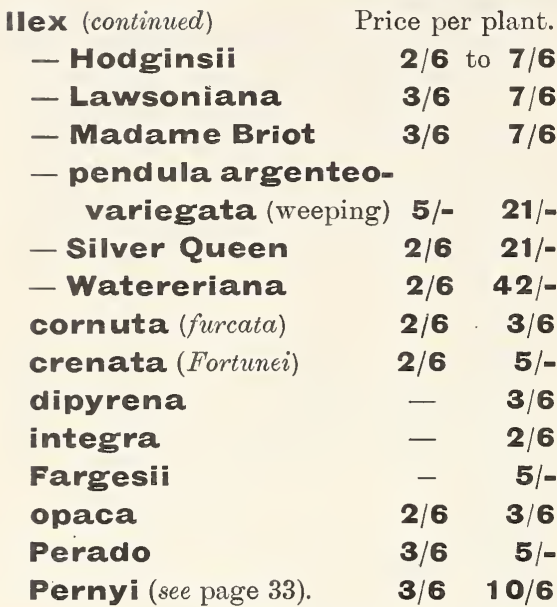

X Gold, Silver, and Green Hollies

in great variety, and of various sizes.

Pyramidal Variegated and Green Hollies, very fine.

Prices on application.

\section{Illicium}

floridanum $\quad 2 / 6$ to $3 / 6$

Laurestinus. See Viburnum p. 40.

$\begin{array}{lll}\text { Laurus } & & \\ \text { nobilis (Sweet Bay) } & 1 / 6 & 5 /- \\ \text { - salicifolia } & 1 / 6 & 2 / 6\end{array}$

Lavandula (Lavender)

Spica (vera)

- alba

9d. $1 /-$

- nana compacta - $1 /-$

\section{Ligustrum (Privet)}

Henryi. Named after

Dr. Henry, the celebrated

Chinese explorer. Neat, attractive, glossy dark green ovate leaves

Ibota

japonicum

- macrophyllum

lucidum

- coriaceum

- tricolor

ovalifolium

* - elegantissimum

6d. $1 /-$

$1 /-\quad 5 /-$

Quihoui

$1 / 6$

* sinense floribundum 1/- to 1/6

* L ovalifolium elegantissimum and sinense floribundum can be supplied as standards, at $\mathbf{3} / \mathbf{6}$ each and upwards.
Ligustrum (continued) Price per plant.

- latifolium robustum $1 / 6 \quad 2 / 6$

strongylophyllum $\quad-\quad 1 / 6$

vulgare sempervirens - $6 d$.

\section{Lonicera}

nitida (see $p .34) \quad$ - $5 /-$

pileata (see $p$. 34) - $1 / 6$

Magnólia

Delavayi.

Large glaucous leaves ;

a very distinct species

from S. China

$10 / 6$ to $21 /=$

grandifiora

Exmouth $3 / 6 \quad 10 / 6$

- ferruginea

$3 / 6 \quad 10 / 6$

For Deciduous kinds see $p .66$.

Mahónia. See Berberis p. 35.

Nandina

domestica $\quad-\quad 2 / 6$

Notospàrtium

australis $\quad-\quad 3 / 6$

$\begin{array}{lll}\text { Carmichaelix } & 2 / 6 & 3 / 6\end{array}$

Olea

Aquifolia ilicifolia,

syn. (Osmanthus Aqui-

folium ilicifolius)

$2 / 6 \quad 3 / 6$

europaa

$-\quad 1 / 6$

excelsa

$2 / 6 \quad 3 / 6$

fragrans

$2 / 6 \quad 3 / 6$

Oleària

argophylla $\quad-\quad 2 / 6$

Forsteri $\quad-\quad 1 / 6$

Gunnii (Eurybia) $\quad-\quad 2 / 6$

Haastii $1 / 6$ to $2 / 6$

macrodonta (ilicifolia) 2/6 $3 / 6$

nummularifolia $\quad-\quad 2 / 6$

$\begin{array}{lll}\text { stellulata } & 2 / 6 & 3 / 6\end{array}$

Oreodàphne

californica (Laurus regalis) $\quad$ 2/6

Osmanthus

$\begin{array}{lll}\text { ilicifolius } & 1 / 6 & 2 / 6\end{array}$

- argenteo. variegatus $1 / 6 \quad 2 / 6$

$\begin{array}{lll}\text { - purpureus } & 1 / 6 & 2 / 6\end{array}$

$\begin{array}{lll}\text { myrtifolius } & 1 / 6 & 2 / 6\end{array}$

Osteomeles

anthyllidifolia $\quad-\quad 3 / 6$

Othéra

japonica (Ilex integra) - $\quad 2 / 6$ 


\section{GENERALAWOLLECTION-continued.}

Ozothàmnus rosmarinifolius $\quad 1 / 6$ to $2 / 6$

Philèsia

buxifolia - 5 /.

Phillyræa

decora

latifolia

$1 / 6 \quad 3 / 6$

$1 / 636$

- ilicifolia $\quad 1 / 6 \quad 3 / 6$

media $1 / 6 \quad 36$

Vilmoriniana (densa) $2 / 6 \quad 3 / 6$

Phiomis

fruticosa

$1 / 6$

Photinia

$\begin{array}{lcc}\text { arbutifolia (dentata) } & 1 / 6 & 2 / 6 \\ \text { Benthamiana } & 26 & - \\ \text { ovata } & 1 / 6 & 2 / 6 \\ \text { serrulata } & 1 / 6 & 2 / 6\end{array}$

Phyllostachys (see $p .43$ ).

Pittosporum

Buchananii

Colensoi

$1 / 6 \quad 2 / 6$

crassifolium (Rulphii) 1/6

$2 / 6$

eugenioides

$2 / 6$

- variegata

$1 / 6 \quad 2 / 6$

Mayanum

$1 / 6 \quad 2 / 6$

Tobira

16

-

undulatum

1626

$2 / 6 \quad 3 / 6$

Prinos

glaber

16

Quércus (Oak)

acuta (Buergeri) (see p. 41).

26

Cerris Lucombeana

(see p.41). $3 / 6 \quad 5 /=$

glabra (latifolia) (see p. 41) $3 / 6 \quad \mathbf{1 0 / 6}$

Ilex (Holly-leaved Ever-

green oak) $1 / 6 \quad 31 / 6$

- Fordii

$2 / 621 /$.

phillyraeoides

$2 / 6 \quad 3 / 6$

Suber (Cork Oak)

$5 /$.

See also Deciduous List, p. 69.

Raphiòlepis

japonica (ovata)

$1 / 6$

2/6

Rhàmnus

Alaternus angustifolius $1 / 6 \quad 2 / 6$ californica
Rosmarinus (Rosemary)

Price per plant.

officinalis, 9/- per doz. - $1 /$.

\section{Ruscus}

aculeatus (Butcher's

$\begin{array}{lll}\begin{array}{l}\text { hypoglossum } \\ \text { racemosus }\end{array} & - & 1 /- \\ \begin{array}{l}\text { (Alexandrian Laurel) } \\ \text { (Aleom) }\end{array} & - & 1 / 6\end{array}$

\section{Sarcococca}

humile. Very dwarf

habit, suitable for dry

soils or planting under

trees. Leaves bright

green, lanceolate and

leathery

ruscifolia. Bright

shining green foliage,

resembling " Butcher's

Broom," compact habit,

thrives under the shade

of trees. Flowers white

and fragrant. A.M.R.H.S. - $\quad 1 / 6$

\section{Senècio}

compacta

elæagnifolius

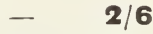

Grayii

$2 / 6$ to $3 / 6$

rotundifolius

- $2 / 6$

Skimmia

Fortunei

$2 / 6 \& 3 / 6$

japonica

$1 /$ - to $2 / 6$

- fragrans

$1 / 6 \quad 8 / 6$

$1 / 6 \quad 2 / 6$

Stranvasia

undulata (see p. 34) - $\quad 3 / 6$

Sycopsis

sinensis. Neat habit,

attaining a height of

10 to 15 feet. The only

species known to be

hardy in this country

$2 / 6$

Thamnocalamus (see $p .43$ ).

Tricuspidaria

hexapetala

$$
\text { (Crinodendron) }
$$

(Hookerianum) - $\mathbf{5 / .}$

dependens (true) $\quad-\quad 3 / 6$

Trochodendron

aralioides. Flowers greenish and rather inconspicuous, although highly interesting. Leaves 4 to 5 inches in length, dark green, glossy and handsome; rapid grower

$5 /=$

Ulex (Furze)

europaus 9/-perdoz. - $1 /$.

- flore pleno $9 /$. , - $1 /$.

strictus $9 / .,, \quad-\quad 1 /$. 


\section{GENERAL COLLECTION-continued.}

Veronica

Price per plant.

Alicia Amherst

*Andersonii

*Autumn Glory

*anomala

*Colensoi glauca

*cupressoides

*Diament

*epacroidea

Gauntletti

Hulkeana. Small lilac-

coloured flowers, borne in

densely-flowered panicles

at the tips of the branches $2 / 6 \quad 3 / 6$

*La Seduisante

*Lindsayii

Macrowia

*pinguifolia

*Purple Queen

*salicifolia (Lindleyana)

*Traversii

$$
\text { * 9/- per dozen. }
$$

For other kinds of Veronicas,

see Herbaceous Catalogue,

free on application.

\section{Viburnum}

\section{Awafukii}

(odoratissimum) -

Viburnum (continued)

Price per plant. coriaceum. Height 12 to 15 feet. Flowers cream-white

Hen ryi. Lanceolate glossy green leaves. Panicles of coral-red fruitsin Autumn, highly ornamental

rhytidophyllum

\section{japonicum} (see p. 34). $\mathbf{3} / \mathbf{6}$ to $\mathbf{2 1 / -}$

Tinus (Laurustinus)

- Iucidum

$1 / 6$ to $5 /=$

- purpureum

$1 / 6 \quad 2 / 6$

$1 / 6 \quad 2 / 6$

utile. Ovate coriaceous leaves, dark glabrous green above and tomentose beneath. White flowers freely produced in terminal corymbs early in April

See also Deciduous List, $p .74$

5/-

$1 /-$

$1 / 6$

$1 /-$

$1 /=$

$1 /-$

1/-

\section{Vinca}

major

- variegata (elegantissima)

6/- per doz.; minor (Periwinkle)

- alba 25/- per 100

- carulea f. pl.

Yucca

filamentosa $\quad 1 / 6$ to $3 / 6$

gloriosa

- variegata

recurva

$5 /-10 / 6$

$5 /-10 / 6$

$2 / 610 / 6$

Whippelii

\section{HARDY ORNAMENTAL GRASSES.}

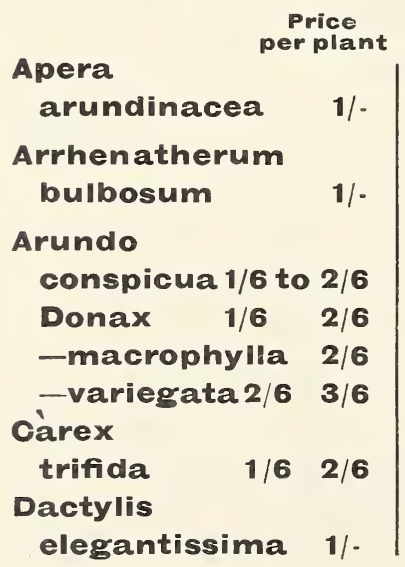

Elymus glaucifolius

Price perplant

Eriànthus

Ravenna

Eulàlia

(Miscanthus) japonica - gracillima

$1 / 6$

-variegata $1 / 6$ to $2 / 6$

-zebrina $1 / 6 \quad 2 / 6$

Glyceria spectabilis

fol. var.
Price

Gymnothrix perplant latifolius $\quad 1 / 6$

Gynerium

(Cortaderia)

argenteum 1/6 to 10/6

-albo-lineata $3 / 65 /=$

-aureo-lineata

Phalaris $3 / 65 /$ (Digraphis)

arundinacea elegantissima 1/Poa aquatica 1/-

\section{Stipa}

gigantea 1/- to 1/6 pennata 1/. $1 / 6$ 


\section{EVERGREEN AND DECIDUOUS OAKS.}

\section{QUERCUS ACUTA (Buergeri)}

A large evergreen shrub or small tree, native of Japan, of spreading habit, with leathery dark green leares, and shoots of olive-coloured bark, which become brown with age.

Either as a single specimen on the lawn or in ornamental plantations this Oak cannot fail to be appreciated.

2/6 each

\section{QUERCUS ALBA}

A large deciduous tree commonly called the American White Oak on account of the bark being white. Leaves oblong, pinnatifidly serrated.

$3 / 6$ each

\section{QUERCUS BANISTERI}

Known also as Quercus ilicifolia and commonly referred to as the Bear Oak. Leaves obovate-wedge-shaped with 3 or 5 deep bristle pointed lobes. A small shrub 3 to 10 feet high introduced from $\mathrm{N}$. America.

$3 / 6$ each

\section{QUERCUS COCCINEA}

(The Scarlet Oak).- In its native home in North America this species forms a tree 70 to 80 feet high, clothed with oblong or oborate deeply lobed leaves, which in autumn assume a brilliant scarlet colour. In his "Silva of North America," Professor Sargent says, "The autumn colours of no other American tree are more splendid, or retain for a longer time their beauty, which is often intensified by the first snowflakes of winter."

\section{QUERCUS CONFERTA}

$3 / 6$ to $10 / 6$ each

This handsome Oak, sometimes known as Quercus pannonica or The Hungarian Oak, is a native of Southern Europe, where it forms an ornamental round-headed tree of mocierate size. The leares are deciduous, almost sessile, oblong-ovate in outline and deeply pinnately lobed. It is a noble tree in its own country, and one of the quickest growing Oaks in cultivation.

$3 / 6$ to $10 / 6$ each

\section{QUERCUS FALCATA}

A deciduous tree 40 to 80 feet in height, remarkable for the great difference in its leaves and general appearance in various climates.

$3 / 6$ each

\section{QUERCUS GLABRA (latifolia)}

A handsome evergreen Oak which it is impossible to recommend too highly. The leaves are from 2 to 5 inches long, and 1 to $1 \frac{1}{4}$ inches broad, glabrous, broadly lanceolate, Laurel-like, dark green above, paler beneath. It is very desirable in its young state as an evergreen shrub and older plants form handsome trees.

$3 / 6$ each

\section{QUERCUS LUCOMBEANA}

Commonly known as The Lucombe Oak or Exeter Oak was raised by Lucombe at Exeter in 1762 , and is supposed to be a hybrid between Quercus Cerris and $Q$ Suber. It makes a handsome sub-evergreen tree, and should find a place in every collection.

\section{QUERCUS NIGRA}

$\mathbf{3} / 6$ and $5 /$ - each

A small deciduous tree 20 to 30 feet high, commonly known as the Black Jack Oak.

Leaves wedge-shaped and slightly three-lobed at the end, deep green in summer and turn

blackish-red in autumn.

$3 / 6$ each

\section{QUERCUS PALUSTRIS}

(The Pin Oak)-In the United States of North America this handsome Oak forms a tree 70 to 80 feet high, with dark green, oborate, sinuately lobed leaves. It is an admirable tree for landscape and park scenery in Great Britain, and is specially valuable for the beauty of its spring and autumn foliage.

$2 / 6$ and $3 / 6$ each

\section{QUERCUS TINCTORIA}

Commonly known as the Dyer's Oak, leares obovate-oblong, dilated, widely serrated. A fine deciduous tree growing to a height of 80 to 100 feet, introduced from the United States. 


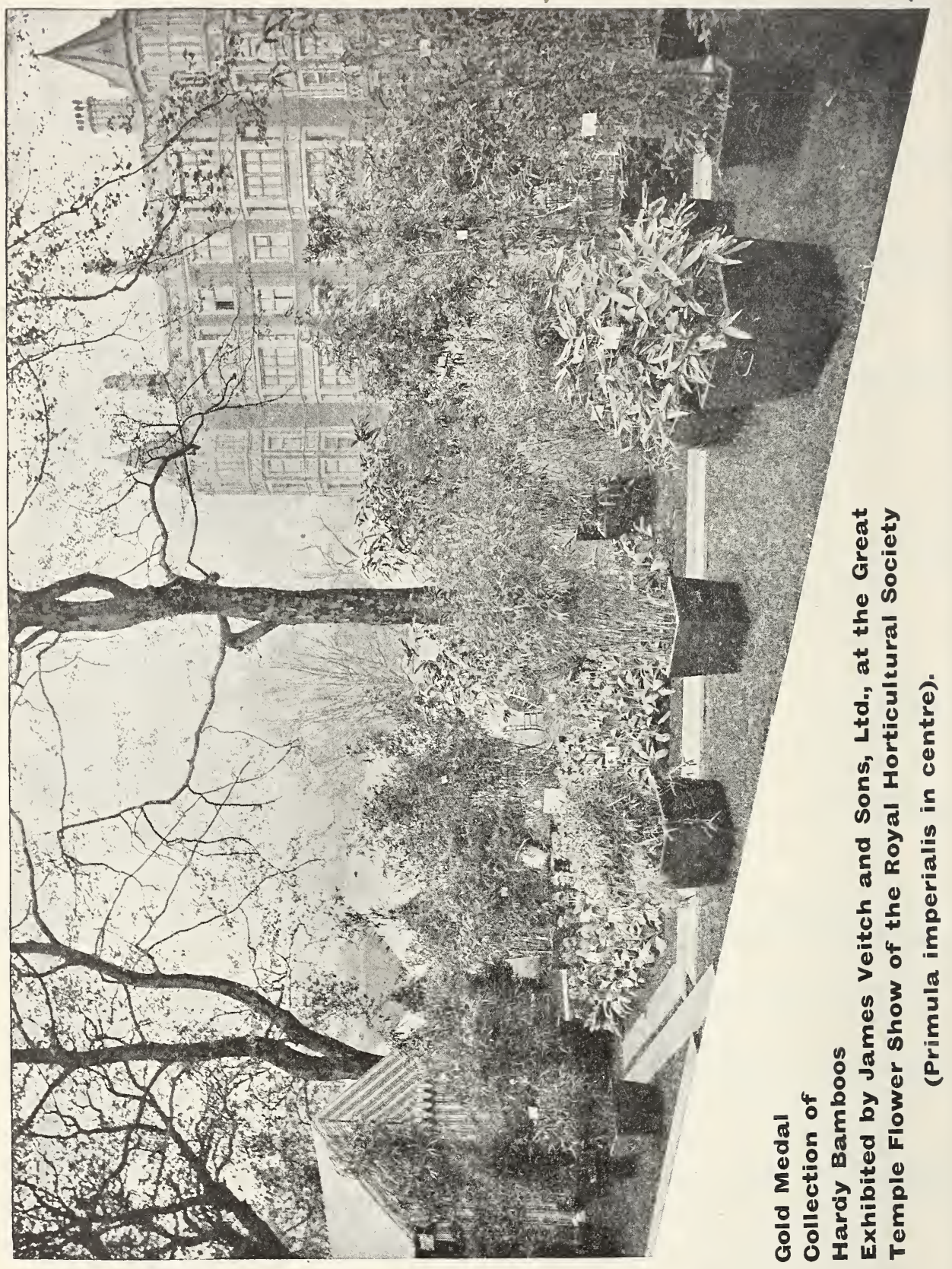




\section{HARDY BAMBOOS.}

The importance of the Hardy Bamboos as subjects for garden decoration is now generally recognised, and to their cultivation, and especially to the introduction of kinds previously unknown in British gardens, we have given especial attention. They now form an interesting feature at our Coombe Wood Nursery.

The Bamboos are one of the most striking characteristics of the tropical vegetation of the Malayan region, whence they spread northwards up the slopes of the Himalayas, and also into China and Japan, where many species occur that are sufficiently hardy for the climate of Britain. By the introduction of these species into British gardens a group of graceful plants of a decidedly tropical type is available for ornamental planting.

The taller Bamboos may be used with the most pleasing effect for the decoration of the lawn, but more especially for the margin of lakes and banks of streams. Their culture is of the easiest description-a retentive soil well supplied with humus or vegetable mould, an abundant supply of moisture, and a sheltered situation. The dwarfer Bamboos are valuable adjuncts to the flower garden, for the margin of beds, for foliage effect interspersed with flowering plants, etc.

Under the general name of Bamboo are included species belonging to several genera as Arundinaria, Bambusa, Phyllostachys, Thamnocalamus, etc.

\begin{tabular}{lcc} 
Arundinaria & \multicolumn{2}{c}{ Each } \\
anceps & $5 /-$ & $10 / 6$ \\
auricoma & $1 / 6$ & $2 / 6$ \\
Fortunei variegata & $1 / 6$ & $2 / 6$ \\
Hindsii & $3 / 6$ & $7 / 6$ \\
- graminea & $3 / 6$ & $7 / 6$ \\
japonica (Metake) & $2 / 6$ & $21 /-$ \\
nitida & $5 / \cdot$ & $21 /-$ \\
pumila & $2 / 6$ & $3 / 6$ \\
Simonii & $2 / 6$ & $10 / 6$ \\
Veitchii (Bambusa) & $2 / 6$ & $5 /-$ \\
Bambusa & & \\
disticha (nana) & $2 / 6$ & $3 / 6$ \\
Laydekeri & $3 / 6$ & $5 /$. \\
Nagashima & $3 / 6$ & $5 / \cdot$ \\
palmata & $2 / 6$ & $5 /-$
\end{tabular}

\begin{tabular}{|c|c|c|}
\hline \multirow{2}{*}{$\begin{array}{l}\text { Bambusa (continued) } \\
\text { pygmæa }\end{array}$} & \multicolumn{2}{|c|}{ Each } \\
\hline & $2 / 6$ & 3 \\
\hline tessellata (Ragamowski) & $2 / 6$ & \\
\hline \multicolumn{3}{|c|}{ Phyllostachys } \\
\hline & $3 / 6$ & 21 \\
\hline & $7 / 6$ & \\
\hline fle: & $5 /-$ & \\
\hline Kumasasa (viminalis) & - & \\
\hline nigra & $5 /-$ & \\
\hline Quilioi & $5 /-$ & \\
\hline violasce & $5 /-$ & \\
\hline viridi-glaucescens & $3 / 6$ & \\
\hline
\end{tabular}

Thamnocalamus

(Arundinaria)

falcata (Bambusa

$\begin{array}{llr} & \\ \text { Falconeri } & 3 / 6 & 5 /-\end{array}$

Other varieties not included in this list can be supplied at current prices. Several of the above-named Bamboos can be supplied in extra-sized plants. Particulars and prices on application. 


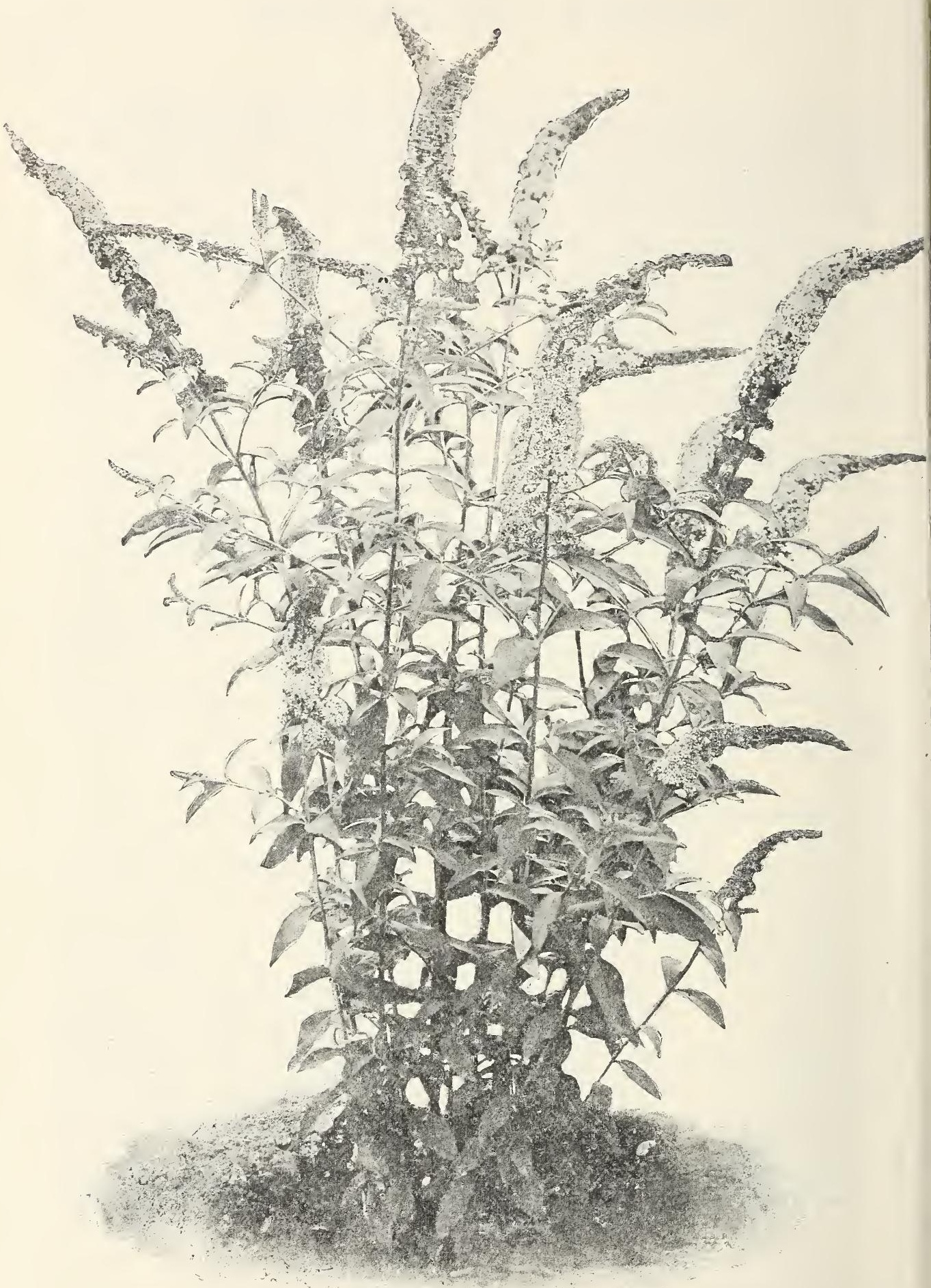

Buddleia variabilis magnifica (see page 45)

$2 / 6$ and $3 / 6$ each. 


\section{NEW AND CHOICE}

\section{DECIDUOUS TREES \& SHRUBS.}

\section{BERBERIS POLYANTHA}

A deciduous shrub 5 to $6 \mathrm{ft}$. high, flowers yellow, followed by coral red fruits. Bright green obovate leaves borne in clusters of about eight each.

$3 / 6$ each.

\section{BERBERIS WILSONAE.}

A fine hardy shrub of dwarf habit with much-branched stems covered with innumerable leaves about $\frac{1}{2}$-inch long. The spines are nearly an inch long and form a protection against birds when the plant is in berry. The flowers are of a rich golden hue, but the superb coral-red berries very freely produced are its chief attraction.

First Class Certificate from the Royal Horticultural Society.

$1 / 6 \& 2 / 6$ each.

\section{BETULA ALNOIDES PYRIFOLIA.}

A deciduous tree, 20 to 40 feet high. Leaves ovate acuminate, resembling those of a Pyrus.

$8 / 6$ each.

\section{BUDDLEIA VARIABILIS MAGNIFICA.}

A truly magnificent variety equal to Teitchiana in size of flower spikes and profusion of bloom whilst the flowers are of a much deeper shade of rose-purple. It thrives well in any soil or situation, and should be well pruned back in spring to ensure blooming in the summer (see illustration p. 44).

$2 / 6 \& 3 / 6$ each.

First Class Certificate from the Royal Horticultural Society.

\section{BUDDLEIA VARIABILIS VEITCHIANA.}

In foliage and habit it resembles the type but is much more robust, the flower spike being denser and much larger. The individual blooms are bright mauve with an orange yellow throat.

$1 / 6 \& 2 / 6$ each.

First Class Certificate from the Royal Horticultural Society.

\section{COTONEASTER APPLANATA.}

$\nabla$ ery distinct and of free growth. The leaves are ovate, dark green above and covered with a grey tomentum beneath. Its berries are brilliant scarlet in colour, produced in great profusion and retained far into the winter months.

1/6 each.

Award of Merit from the Royal Horticultural Society.

\section{COTONEASTER REFLEXA.}

A free-growing deciduous species with ovate leaves, particularly suitable for covering a wall. Pure white flowers freely produced in May. (See illustration p. 88). 1/6 each.

\section{DAVIDIA INVOLUCRATA}

A tree that reaches the height of 30 feet, with leaves resembling those of the Lime. The remarkable feature of this introduction is its large pure white bracts produced in May which afford a conspicuous contrast to the dark green foliage. Flowered at Coombe Wood in May 1911 (first time in England). See illustration p. 46. 7/6 to 21/- each. 


\section{EUCRYPHIA PINNATIFOLIA.}

A remarkably handsome and distinct deciduous shrub with rose-like foliage. The flowers are from $2 \frac{1}{2}$ to 3 inches in diameter, each with four pure white spreading petals and numerous stamens, with long filaments terminating in bright golden yellow anthers. They resemble in form an Hypericum, and are produced in July and August. The plant has withstood many severe winters uninjured at our Coombe Wood Nursery.

First Class Certificate from the Royal Horticultural Society.

$5 /-\& 10 / 6$ each.

\section{FRAXINUS BRACTEATA}

A deciduous tree of neat habit with light elegant pinnate foliage, deep glossy green above, bright green beneath. Height 40 feet. Discovered near Ichang, China

5 - each.

\section{haMAMELIS MOLLIS.}

A very remarkable hardy Witch Hazel, the leaves of which are larger than any other. The strap shaped petals are about $\frac{3}{4}$ inch long, narrow and wavy, and of a rich orange-yellow colour, whilst the calyx lobes are deep chocolate.

$3 / 6$ and $5 /$-each.

\section{HIPPOPHAË RHAMNOIDES.}

This, during the late autumn and winter months, when covered with its orange-red fruits, is one of the most ornamental fruiting shrubs our gardens possess, and yet, owing to an insufficient knowledge of its floral structure, planters often fail to secure a crop. The plants are diœcious-i.e., the male and female flowers are produced on different plants, and it is useless planting one sex without the other. We have propagated a large quan. tity of carefully selected plants, both male and female.

Male plants, 1/-; Female plants, 9d. each.

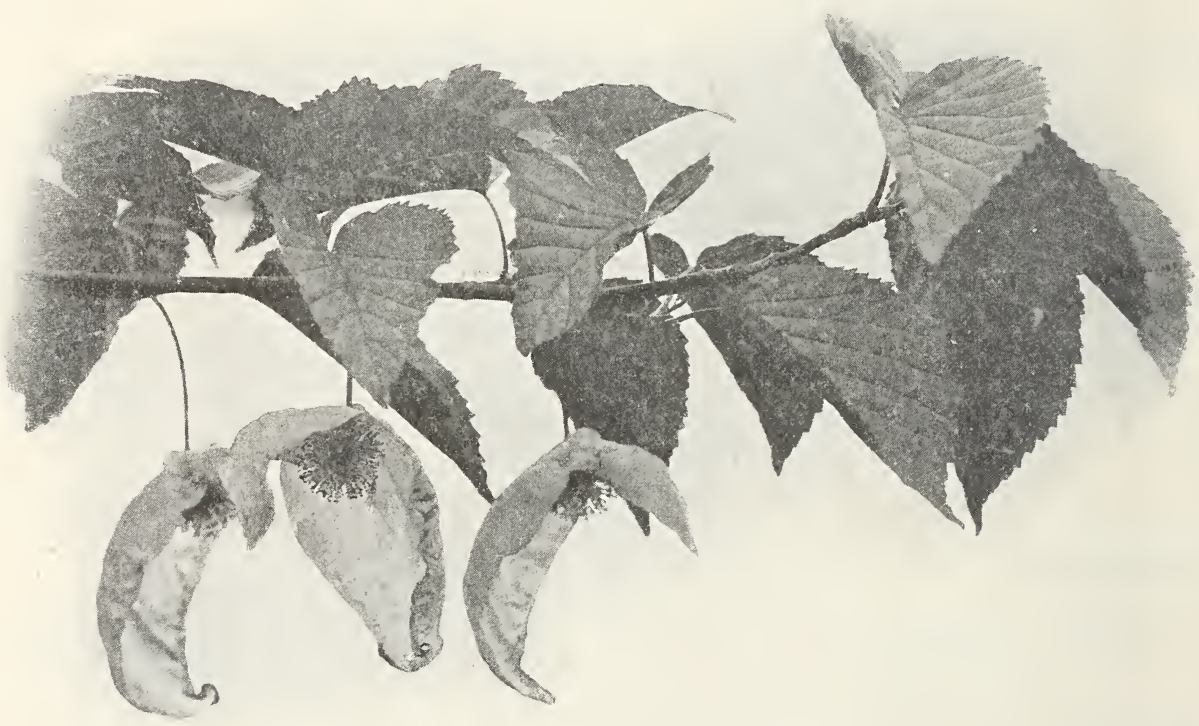

Davidia involuctata (see page 45).

Photographed at Coombe Wood in May, 1911, the first time of flowering in England. 
NEW AND CHOICE DECIDUOUS TREES \& SHRUBS (continued). 47

\section{JUGLANS CATHAYENSIS.}

A new species of Walnut with large dark green pinnate leaves, rivalling those of Ailantus, leaflets oval. A handsome ornamental deciduous tree suitable for Parks or gardens. (See illustration below.

106 each

Illustrated in Gard. Chron. Sept. 9th, 1911.

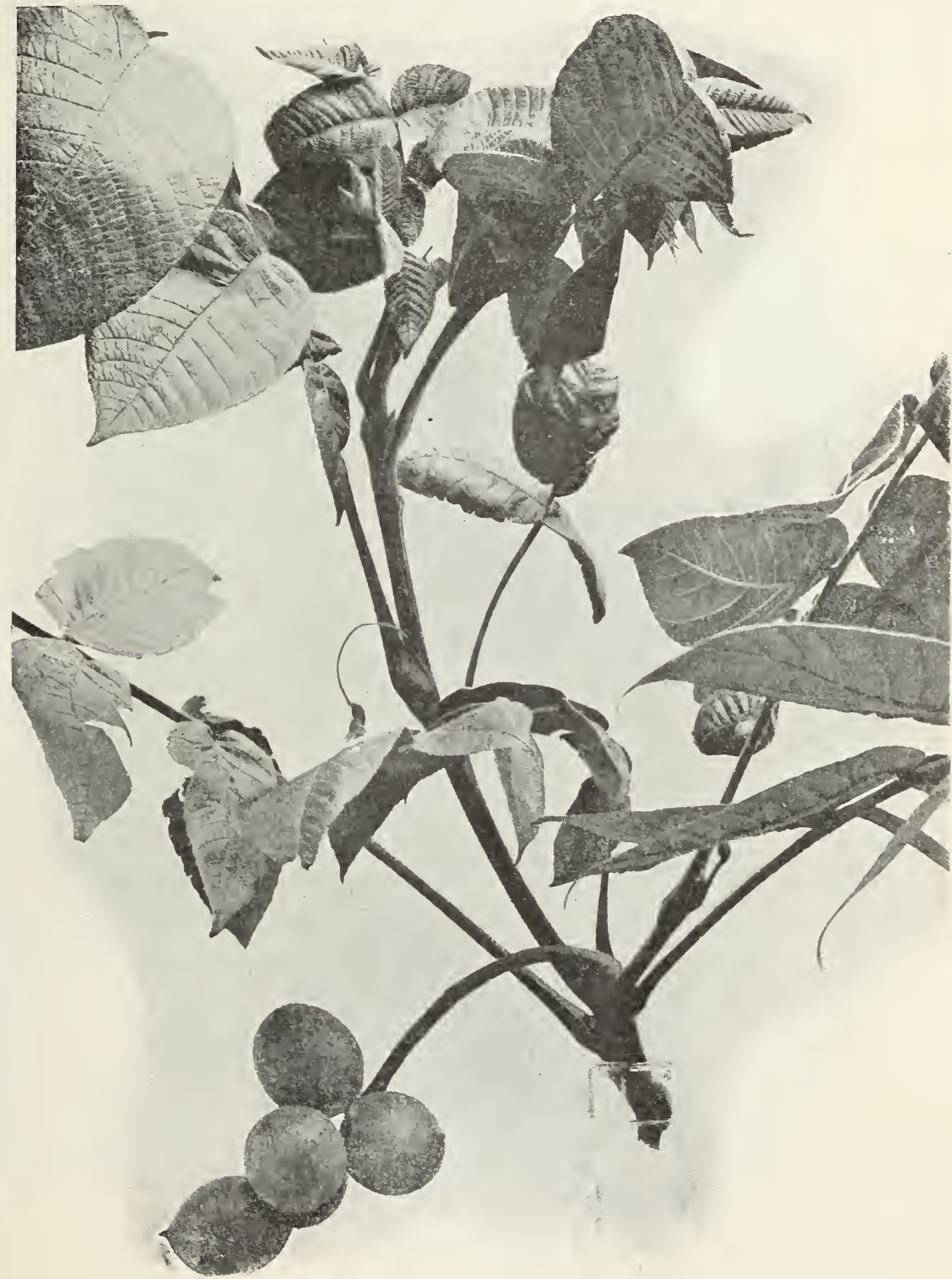

Juglans Cathayensis (see description above). 


\section{DECIDUOUS TREES AND SHRUBS (continued).}

\section{MAGNOLIA PARVIFLORA.}

A deciduous shrub with roundish-oval leaves. The fragrant flowers are pure white with a central disc of deep claret-coloured stamens, a very striking contrast (see illustration below).

$7 / 6$ and $10 / 6$ each

\section{MAGNOLIA WATSONI.}

Fragrant cream-white flowers about 5 or 6 inches across; leaves oblong obovate, about six inches long.

7/6 and 10/6 each

\section{POPULUS LASIOCARPA.}

The ovate cordate leaves of this new Poplar are extremely large and attractive, measuring 10 to 12 inches in width. They are bright green in colour, whilst the petiole, midrib and principal veins are of a rich red hue.

First-class Certificate from the Royal Horticultural Society.

10/6 each

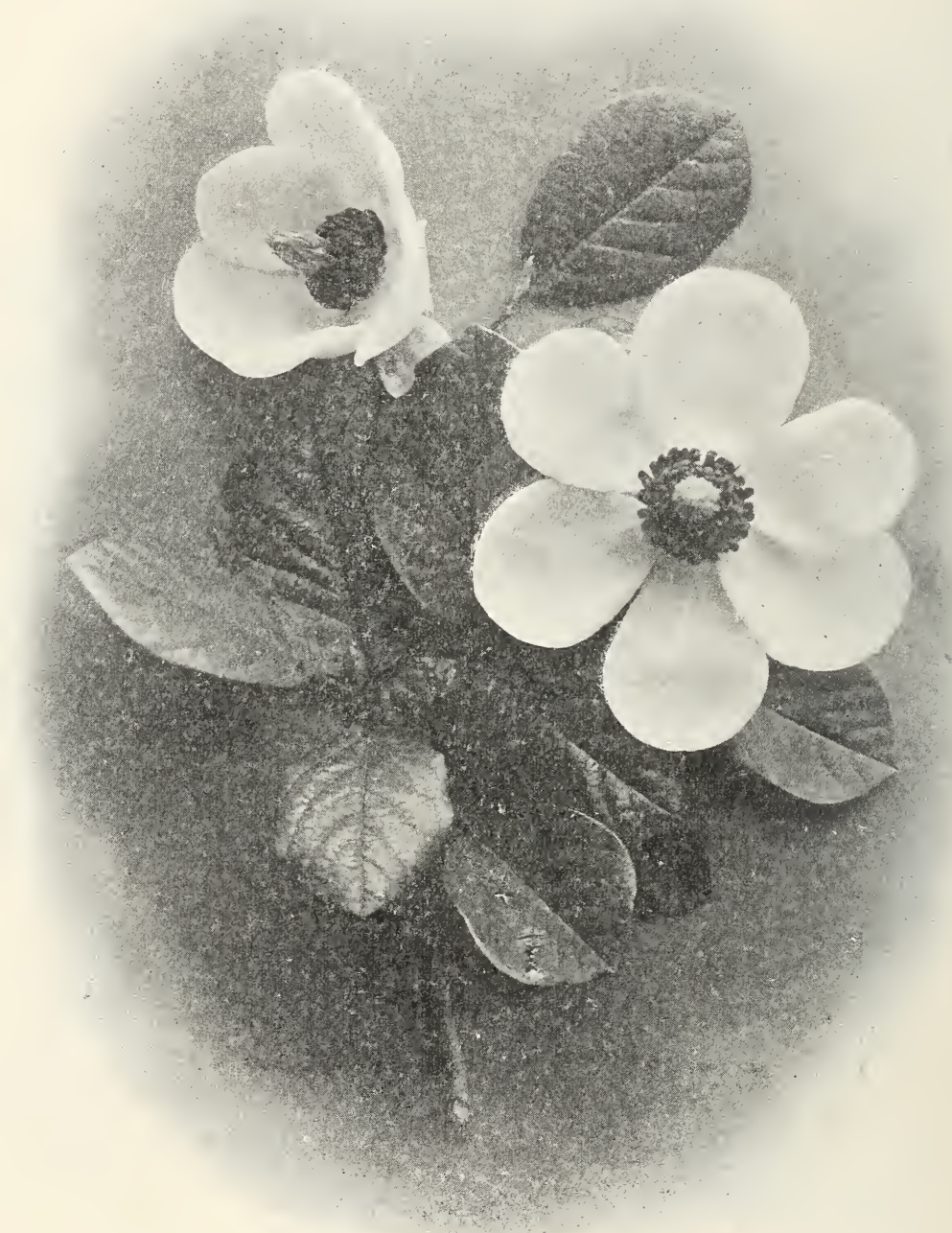

Magnolia parvifiora (see description above). 


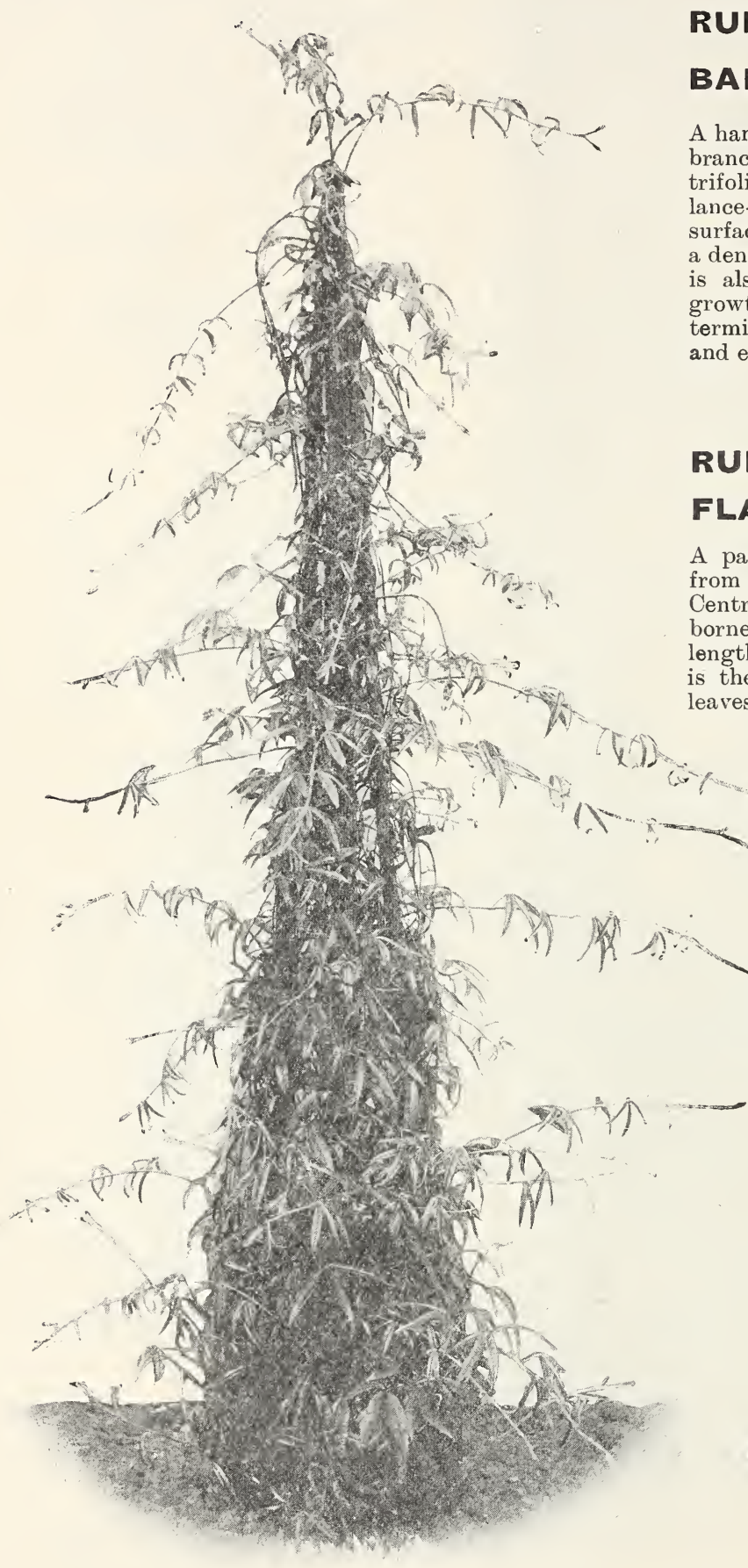

Rubus bambusarum.

\section{RUBUS}

\section{BAMBUSARUM.}

A handsome bramble, with trailing branches 10 to 12 feet long, having trifoliate leaves. The leaflets are lance-shaped, glabrous on the upper surface and covered beneath with a dense dun-coloured tomentum, as is also the wood of the young growths. The flowers are in terminal racemes, the fruits black and edible. 2/6 \& $3 / 6$ each.

\section{RUBUS \\ FLAGELLIFORMIS.}

A particularly handsome species from the mountain woods of Central China, with white flowers borne on growths 6 to 8 feet in length. The attractive feature is the metallic appearance of the leaves which are cordate, acuminate with an irregularly serrate margin. The under surface of the leaf is covered with a thick dun-coloured tomentum, as are also the current year's growths. A very useful addition to our hardy climbing plants (see illustration p. 51) 2/6 \& $3 / 6$ each

\section{RUBUS INNOMINATUS.}

The stems of this handsome bramble are covered with a curiously soft pubescence, giving a velvety impression when touched. The leaves are usually trifoliate, dark green on the upper surface and greyish - white beneath. The edible orange scarlet fruits are borne in panicles 18 inches long, and form a charming ornament to the garden in September.

\section{RUBUS VEITCHII.}

A handsome deciduous shrub with pinnate leaves. Flowers rosy-purple succeeded by red globose fruits. The stems are pubescent and of ornamental value during the winter.

5/- each. 
NEW AND CHOICE DECIDUOUS TREES AND SHRUBS.

51

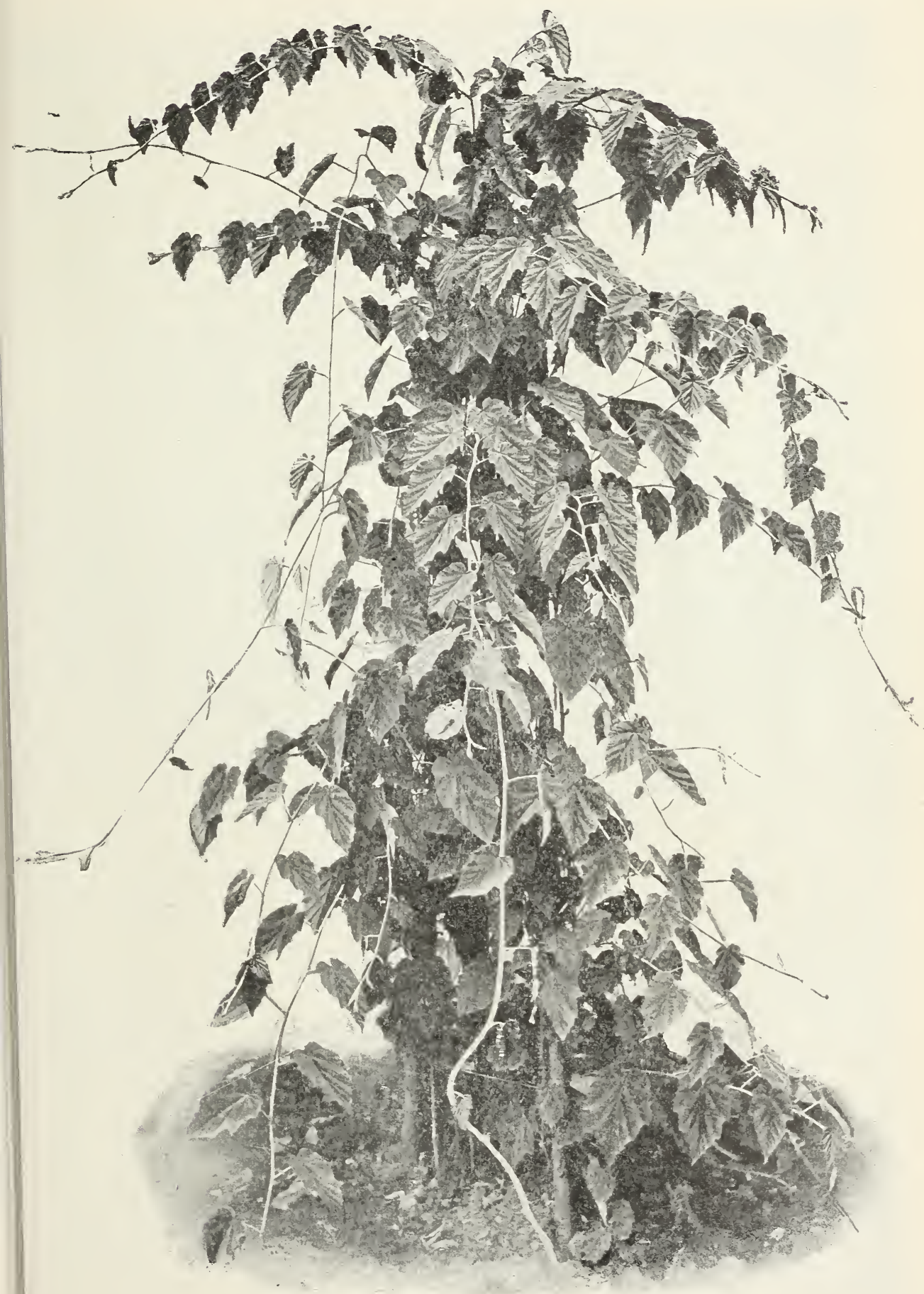

Rubus flagelliformis (see page 50) 


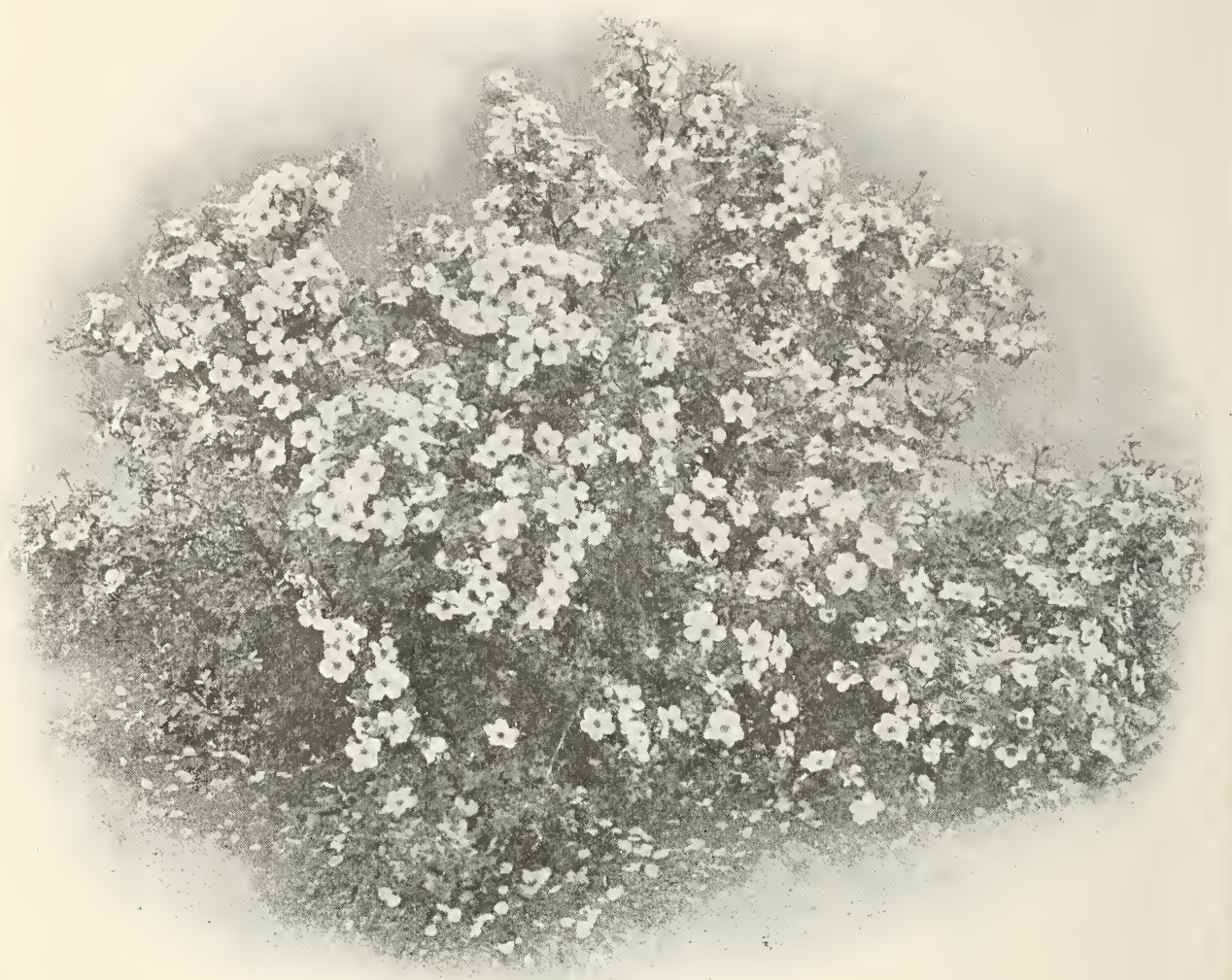

\section{POTENTILLA VEITCHII.}

Neat rounded habit, growing 3 to 4 feet high, producing sprays of snow-white flowers from May till late Autumn, raised well above the grey-green foliage. A charming free-flowering species. See illustration above.

$3 / 6$ each

\section{SPIRAEA HENRYI.}

Numerous rounded corymbs of white flowers, borne on long arching growths of the previous season. June-flowering. Spreading habit 7 to 8 feet high. See illustration p. 53 . Figured in Botanical Magazine T. 8270. 


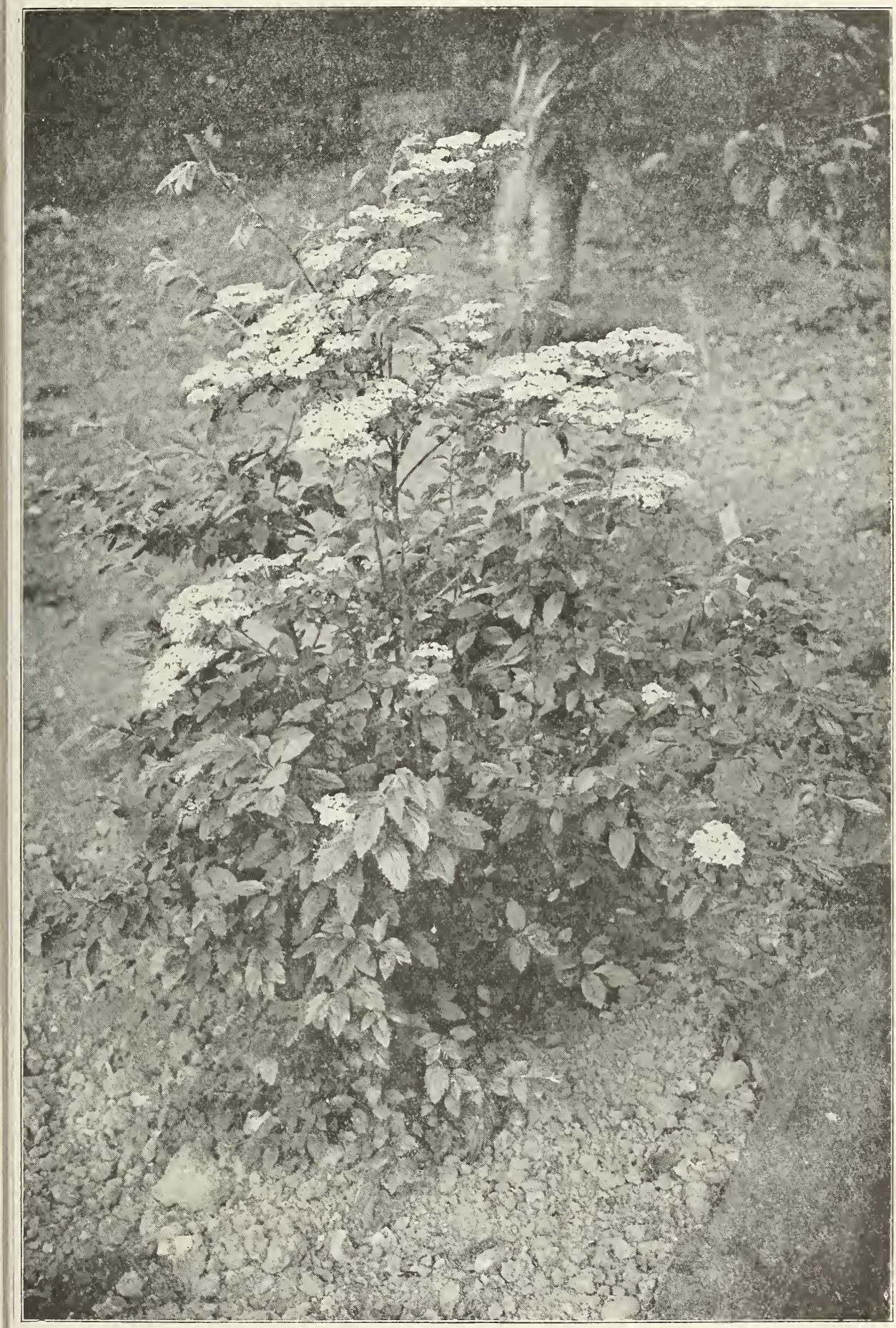




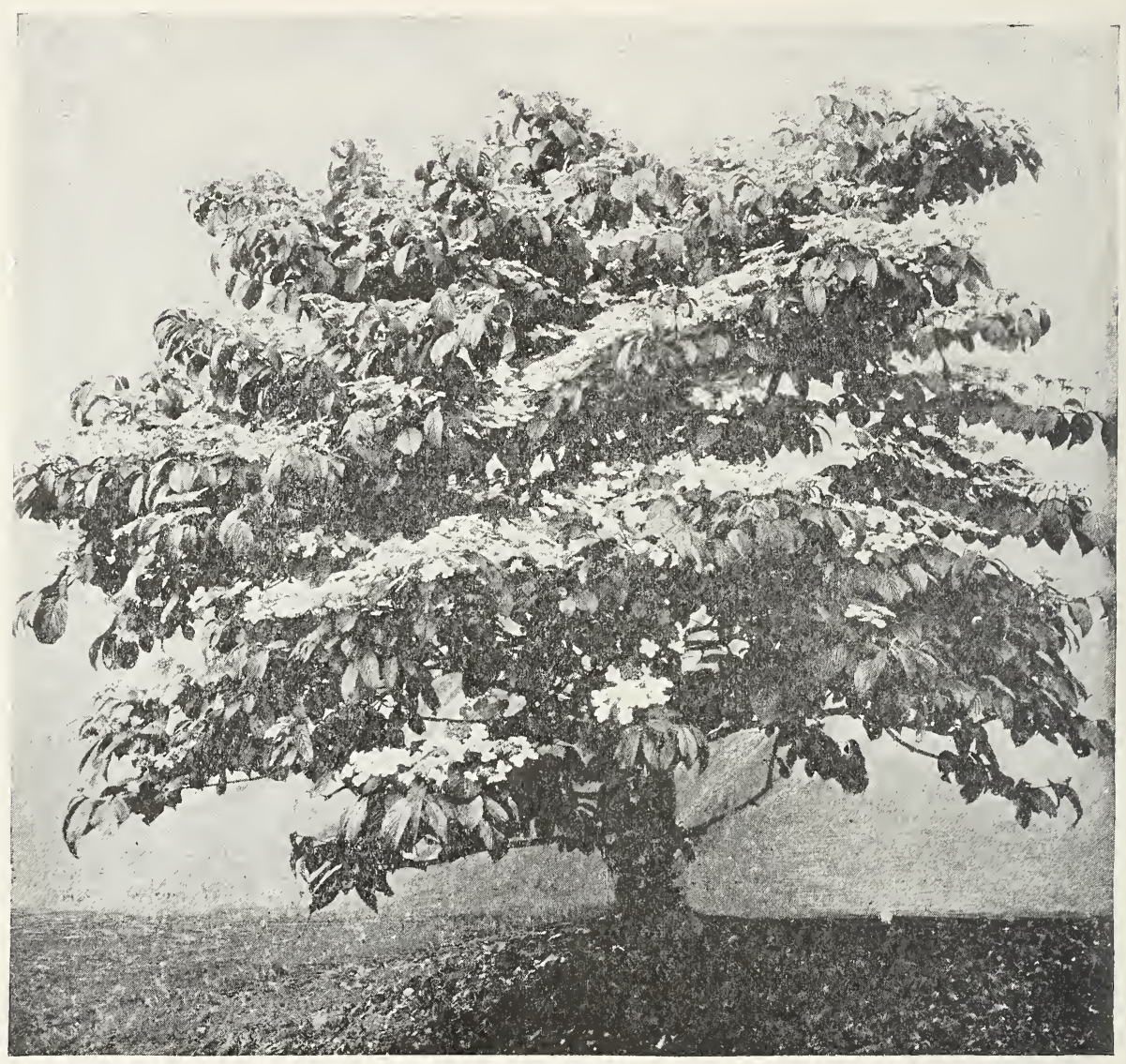

\section{VIBURNUM TOMENTOSUM MARIESII.}

A very handsome shrub of graceful spreading habit, closely allied to the Japanese Viburnum plicatum. The white flowers are produced in great abundance during May. See illustration above. 


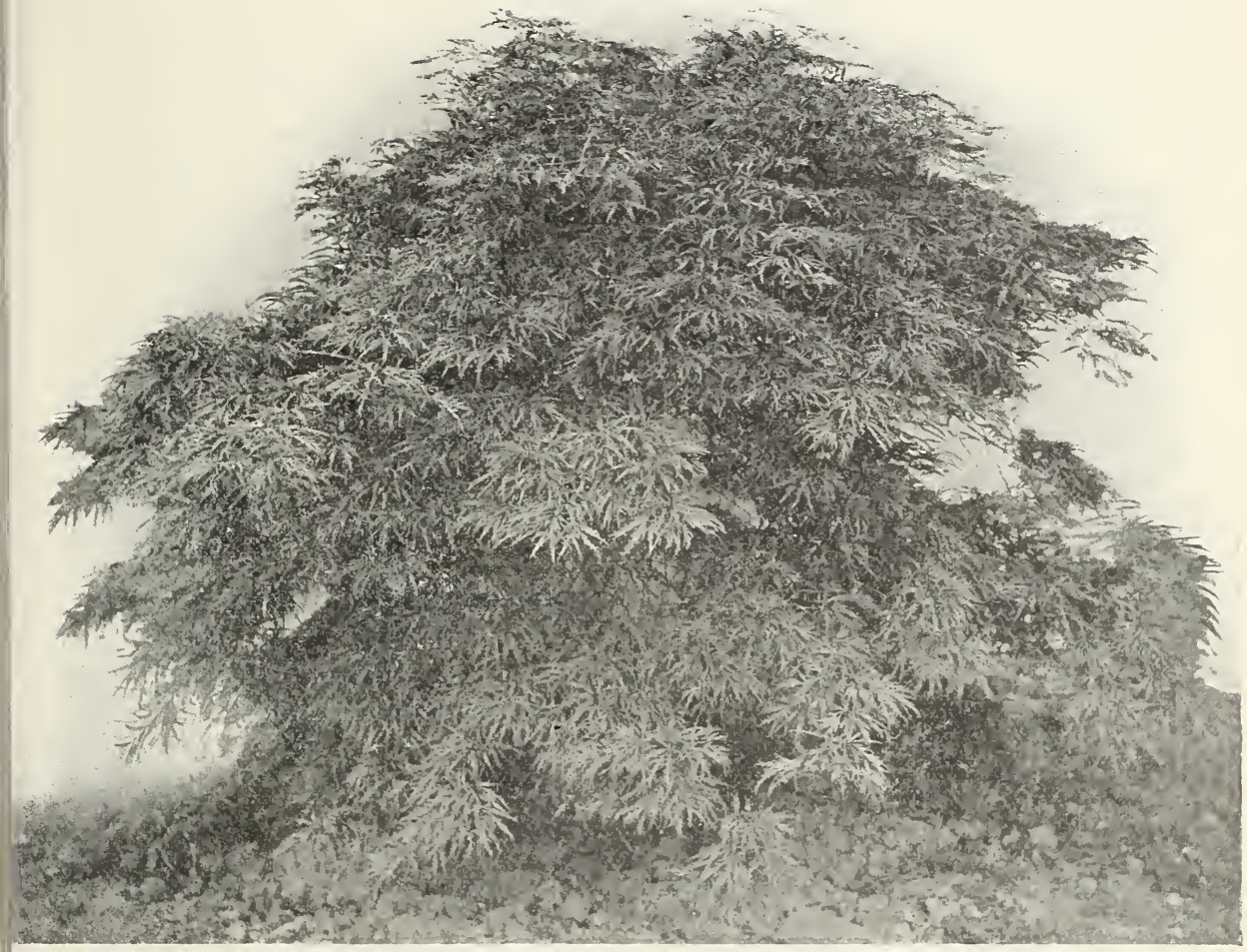

Acer palmatum palmatifidum.

A Japanese Maple with elegant finely-cut dark green foliage.

\section{DECIDUOUS TREES AND SHRUBS.}

\section{GENERAL COLLECTION.}

Abèlia

\section{foribunda}

rupestris (chinensis)

serrata (uniflora)

triflora

\section{Abutilon}

\section{vitifolium}

- album
Price per plant.

26 to $3 / 6$

$1 / 62 / 6$

- $2 / 6$

$1 / 6 \quad 2 / 6$

$2 / 6 \quad 3 / 6$

$2 / 6 \quad 3 / 6$

Acacia. See Robinia. page 70

Acer (Maple)

Japanese species and varieties.

$\begin{array}{lll}\text { argutum } & 2 / 6 & 3 / 6 \\ \text { carpinifolium } & 2 / 6 & 3 / 6 \\ \text { cratagifolium (Veitchii) } & - & 3 / 6 \\ \text { diabolicum (pulchrum) } & - & 2 / 6 \\ \text { distylum } & - & 3 / 6\end{array}$

Price per plant.

Acer (continued) japonicum

- aureum

- laciniatum

$3 / 6$ to $5 /=$

$36 \quad 10 / 6$

$36 \quad 10 / 6$

- microphyllum _ $\quad 3 / 6$

$\begin{array}{lll}\text { palmatum } & 3 / 6\end{array}$

- ampelopsifolium $2 / 6 \quad 3 / 6$

- atropurpureum $\quad 2 / 6 \quad 3 / 6$

- aureum _ $3 / 6$

- decompositum $2 / 6 \quad 3 / 6$

$\begin{array}{lll}\text { - dissectum } & 2 / 6 & 10 / 6\end{array}$

- flavescens $\quad 2 / 6 \quad 3 / 6$

$\begin{array}{lll}\text { - involutum } & 2 / 6 & 3 / 6\end{array}$

- linearilobum _ 3/6

$\begin{array}{lll}\text { - - atropurpureum } & 2 / 6 & 10 / 6\end{array}$

$\begin{array}{lll}\text { - palmatifidum } & 3 / 6 & 10 / 6\end{array}$

(See illustration above.) 


\section{GENERAL COLLECTION (continued)}

Acer (continued) Pric
palmatum roseo-
marginatum
- sanguineum
- septemlobum
- - elegans
- - - purpureum
rufinerve
Other species and varieties.
campestre
circinatum
colchicum aureum
A golden-coloured form
of Acer pictum, frequently
met with in gardens under
the name of A. colchicum.

$\begin{array}{lr}3 / 6 & \text { to } 5 /= \\ 3 / 6 & 10 / 6 \\ 2 / 6 & 3 / 6 \\ - & 3 / 6 \\ 3 / 6 & 5 /- \\ 2 / 6 & 3 / 6\end{array}$

- $1 /=$

$3 / 65 /-$

$3 / 65 /=$

$\begin{array}{lrr}\text { - rubrum } & 1 / 6 & 5 /- \\ \text { creticum (heterophyllum) } & 2 / 6 & 3 / 6\end{array}$

dasycarpum(eriocarpum)2/6 $\mathbf{5} / \mathbf{-}$

fraxinifolium. See Negundo. page 66

Ginnala

$1 / 6 \quad 3 / 6$

monspessulanum $\quad 1 / 6 \quad 2 / 6$

pensylvanicum

platanoides

$\begin{array}{lll}\text { (striatum) } & \mathbf{2} / 6 \quad 3 / 6\end{array}$

(Norway Maple) 2/6

$5 /-$

- aureo-variegatum 2/6

$3 / 6$

- dissectum

$3 / 6$

- laciniatum

(Hawk's-foot Maple)

$2 / 6$ to $5 /=$

- Reitenbachii

$2 / 6$

- Schwedleri

$2 / 6$

Pseudo-platanus

(Sycamore) 1/6

- atropurpureum 2/6

$6 \quad 3 / 6$

- Leopoldii

$2 / 6$

- Prince Handjery

$2 / 6$

- Worlei

$2 / 6$

rubrum (Scarlet Maple) -

saccharinum

Semenovii

(Sugar Maple) 1/6

$2 / 6$

$1 / 6$

tartaricum

AEsculus (Horse Chestnut)

Hippocastanum

$$
\text { (common) } 1 / 6
$$

- album flore pleno $1 / 6$

- laciniata

rubicunda (carnea)

$$
\text { flore pleno }
$$

- Briotii

(Pavia) flava (octandra) 2/6

- macrostachya

- rubra

$$
\text { (parviflora) 2/6 }
$$

- sinensis

$5 /-$

$3 / 6$

$3 / 6$

$5 /=$

$2 / 6$

$2 / 6$

$3 / 6$

$2 / 6$

\section{Ailanthus}

flavescens

(Cedrela sinensis) $\quad \mathbf{1 / 6}$ to $\mathbf{2 / 6}$ glandulosa (Tree of

$\times$

$$
\text { Heaven) 1/6 5/= }
$$

Alnus (Alder)

cordifolia (cordata) $\quad 1 / 6 \quad 2 / 6$

firma multinervis $\quad 2 / 6 \quad 3 / 6$

glutinosa (commonAlder)6d. $\quad 1 / 6$

- crispa $\quad 2 / 6 \quad 3 / 6$

- foliis aureis $\quad 3 / 6 \quad 5 /=$

- laciniata $\quad 1 / 6 \quad 2 / 6$

_ imperialis $\quad 2 / 6 \quad 3 / 6$

$\begin{array}{lll}\text { nitida } & 2 / 6 & 3 / 6\end{array}$

Althea Frutex. See Hibiscus. page 65

Amelanchier(SnowyMespilus)

arbutifolia

grandiflora $1 / 6$ to $2 / 6$ canadensis (Botryapium) 1/6 2/6

- forida

$1 / 6 \quad 2 / 6$

ovata

$1 / 6 \quad 2 / 6$

vulgaris

$1 / 6 \quad 2 / 6$

\section{Amorpha}

canescens (Lead Plant) - $1 / 6$

fruticosa (False Indigo) — $1 / 6$

Amygdalus (Almond)

communis amara

(Bitter Almond)

Davidiana alba

$2 / 6$

- rubra

$1 / 6 \quad 2 / 6$

dulcis (Sweet Almond)

$1 / 6 \quad 2 / 6$

$\begin{array}{lll}\text { - macrocarpa } & 1 / 6 & 2 / 6\end{array}$

- purpurea $\quad 2 / 6 \quad 3 / 6$

nana

$1 / 6 \quad 2 / 6$

\section{Persica flore pleno}

(double-flowering Peach)

$\begin{array}{lll}- \text { crimson } & 2 / 6 & 5 /= \\ - \text { magnifica } & 2 / 6 & 5 /=\end{array}$

The best flowering Peach.

Brilliant semi-double car-

mine-crimson flowers.

F.C.C.R.H.S.

\section{- - rose}

The varieties of Amygdalus persica flore pleno are admirable plants for conservatory decoration early in the season, requiring but little forcing. 


\section{GENERAL COLLECTION (continued)}

Aràlia

hybrida

Price per plant.

mandschurica

(Dimorphanthus) $\quad 2 / 6 \quad 36$

- fol. argenteo-variegata

A remarkably handsome foliage shrub, formerly known as Dimorphanthus. Useful for sub-tropical gardening.

\section{- - aureo-variegata 10621}

Foliage variegated with golden yellow, an admirable companion to the Silver-variegated form.

\section{Maximowiczii \\ pentaphylla \\ spinosa (Acanthopanax}

$26 \quad 36$ japonicum

Aronia

floribunda

1626

Artemisia

Abrotanum (Southern-

$$
\text { wood) }
$$

\section{Atriplex}

\section{canescens}

Halimus

Benthàmia

japonica. See Cornus $\mathbf{3} / \mathbf{6} \quad \mathbf{5} /$ Kousa, page 59.

Be'rberis (Barberry)

$\begin{array}{lcl}\text { amurensis } & - & 1 / 6 \\ \text { aristita } & - & 1 / 6 \\ \text { asiatica } & - & 1 / 6 \\ \text { concinna } & - & 16 \\ \text { elegans } & - & 2 / 6 \\ \text { polyantha (see page } 45) . & - & 3 / 6 \\ \text { Thunbergii } & 1 /- & 16 \\ \text { vulgaris (common) } & 1 /- & 1 / 6 \\ \text { - atropurpurea } & 1 /- & 1 / 6 \\ \text { - virescens } & - & 1 / 6 \\ \text { Wilsonze (ste page } 45 \text { ). } & 1 / 6 & 2 / 6\end{array}$

For Evergreen Species, see page 35.

Bètula (Birch)
alba (common)
6d. 26
- fastigiata
16
26
- laciniata pendula
- latifolia
$3 / 6 \quad 5$.
16226
- pendula Youngii
3676
2636
- purpurea

Bètula (continued)

Price per plant.

$$
\text { lenta }
$$

Maximowiczii

$1 / 6$ to 26

papyrifera

populifolia

36

$1 / 6$

rotundifolia (pumila) $\quad$ 2/6

Broussonètia

dissecta (papyrifera

papyrifera

$2 / 6$

$2 / 6 \quad 3 / 6$

\section{Buddleia}

albifiora

Pale mauve flowers with an orange-yellow throat.

Colvilei

$2 / 6 \quad 3 / 6$

A handsome Himalayan species, but not sufficiently hardy for every situation. Should be planted against a south or south-west wall, with slight protection during very severe weather. Bright rose-pink campanulate flowers, produced in long terminal panicles.

globosa

$1 / 6$

intermedia

Lindleyana

variabilis magnifica

(see page 45).

- Veitchiana (see

page 45)

$2 / 6 \quad 3 / 6$

\section{Cæsalpinia}

japonica

$1 / 6 \quad 2 / 6$

Spreading habit, moderate height, stems and branchesarmed with numerous hard curved prickles and furnished with foliage of soft and pleasing aspect; the raceme bears from twenty to thirty bright canary-yellow flowers, an inch in diameter, red filaments and anthers. F.C.C., R.H.S.

\section{Calophaca}

wolgarica

Calycánthus (American Allspice)

floridus $1 / 6$ to $2 / 6$ occidentalis

(macrophyllus)

præcox (Chimonanthus fragrans) 


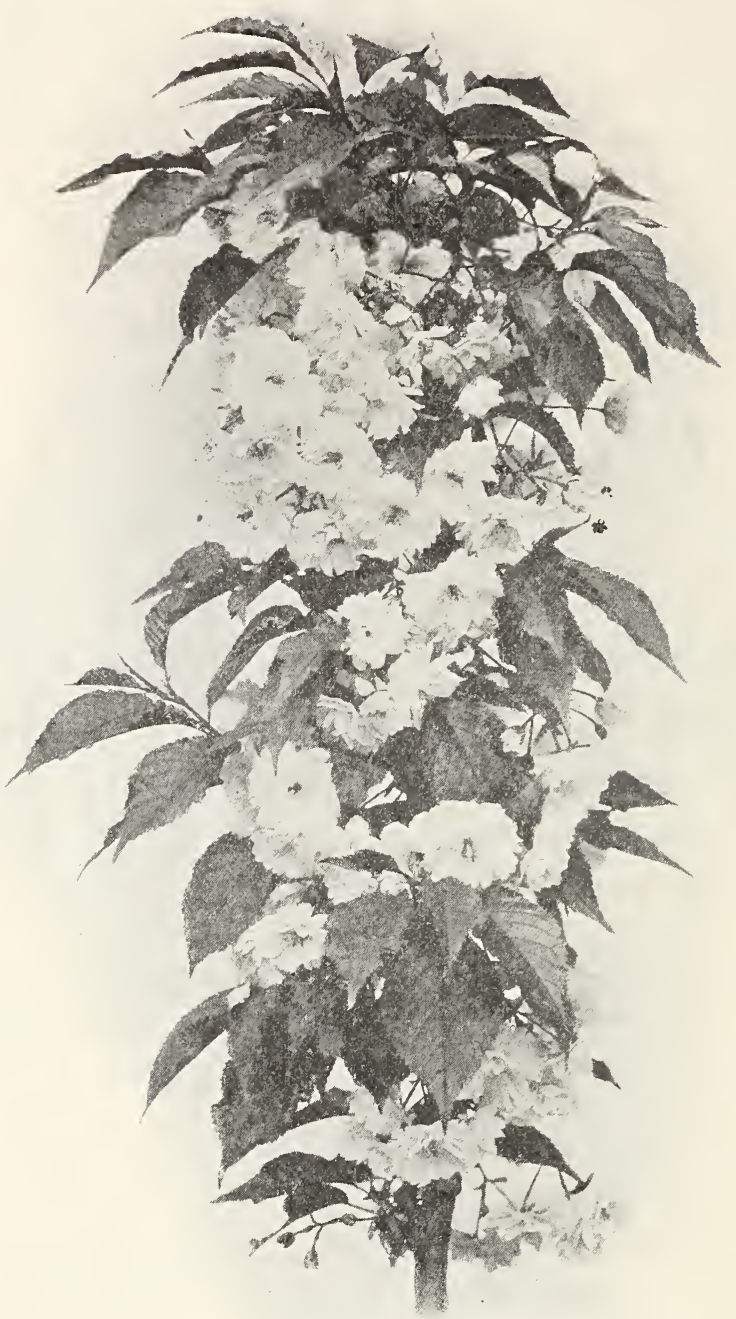

Cerasus Pseudo-cerasus James H. Veitch.

The best double rose Japanese Cherry, flowering in May. Very useful for forcing purposes (see page 59).

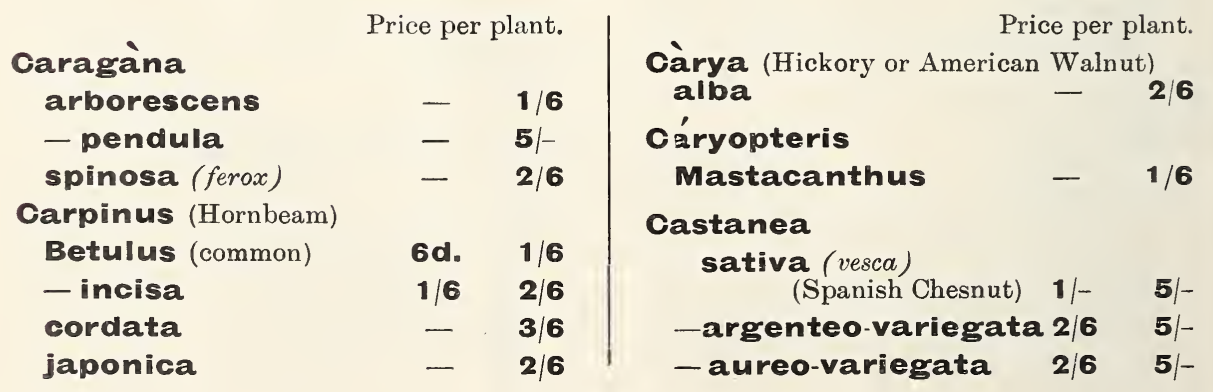




\section{GENERAL COLLECTION (continued).}

\section{Càtalpa}

Price per plant.

\section{bignonioides}

(syringafolia)

$\mathbf{2} / \mathbf{6}$ to $\mathbf{5} /-$

- aurea (useful for subtropical gardens)

$1 / 6 \quad 5 /-$

- purpurea

$2 / 6 \quad 3 / 6$

japonica

Quick grower, fragrant

flowers, very freely produced.

\section{Kampferi}

$-\quad 3 / 6$

speciosa (cordifolia) $3 / 6 \quad 5 /-$

Cèdrela

sinensis (Ailanthus flarescens)

\section{Celastrus} latifolius

Large broadly ovate dark green leaves. Flowers greenish-white borne in terminal panicles

Cephalanthus occidentalis (Button Wood) 2/6

Cèrasus (Cherry)

Avium (Gean)

- Juliana pendula $3 / 6$

- Iatifolium pendula $3 / 6$

- multiplex

Mahaleb

- pendula

- variegata

Padus (Bird Cherry) $16 \quad 26$

Pseudo-cerasus-

(C. Watereri) ) dwarfs $16 \quad 2 / 6$ (C. Sieboldii) $\{$ standards $2 / 6$ to $3 / 6$

_ James H. Veitch-

$\begin{array}{lll}\text { dwarfs } & \mathbf{2} / \mathbf{6} & \mathbf{3} / \mathbf{6} \\ \text { standards } & \mathbf{3 / 6} & \mathbf{5} /-\end{array}$

(See illustration $p .58$ )

The above are the two finest double rose forms of the Japanese Cherry.

\section{semperflorens}

(All Saint's Cherry)

$\begin{array}{ll}1 / 6 & 2 / 6 \\ 3 / 6 & 5\end{array}$

serotina

$3 / 65$ -

serrulata (See illus-

$$
\text { tration) p. 60)- }
$$

$\begin{array}{rcc}\text { dwarfs } & 1 / 6 & 2 / 6 \\ \text { standards } & - & 3 / 6\end{array}$

The best double white Japanese Cherry.

\section{sinensis pendula}

\section{rosea-}

standards

A very beautiful weep ing form of single pink Japanese Cherry.

\section{Cercidiphyllum}

japonicum

Price per plant.

\section{Cercis}

canadensis

Siliquastrum (Judas

- alba

$2 / 6$ to 5 -

$263 / 6$

\section{Chimonanthus}

fragrans (Calycanthus

$$
\text { procox) } \mathbf{1} / \mathbf{6} \quad \mathbf{2} 6
$$

\section{Chionanthus}

retusus (See illustration p.61) $\quad 3 / 6$

virginica (Fringe Tree) $16 \quad 2 / 6$

\section{Cladrastis}

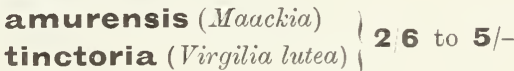

\section{Clerodéndron}

trichotomum

$26 \quad 36$

Colutea

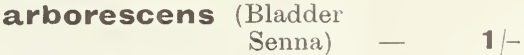

cruenta $\quad-\quad 1 / 6$

melanocalyx (longialata) - 16

Comptónia (Myrica)

asplenifolia

Cornus (Jogwood)

alba

6d. 1

- atrosanguinea

- 16

- Späthii

1626

- variegata

1626

alternifolia

$2 / 6$

brachypoda

$2 / 6$

- variegata

circinata

$162 / 6$

florida

$2 / 6 \quad 3 / 6$

- rubra

Kousa (Benthamiajaponica) $\mathbf{3} 6 \quad \mathbf{5}$ -

Perfectly hardy and flowering as freely as other Dogwoods when it has been established a few years. F.C.C., R.H.S.

macrophylla

Mas

- elegantissima

$\begin{array}{lcc}\text { - variegata } & 1 / 6 & 2 / 6 \\ \text { Nuttallii } & - & 5 /- \\ \text { sanguinea } & -/ 6 & 1 /- \\ \text { stolonifera } & 1 /- & 1 / 6 \\ \text { stricta } & 1 / 6 & 2 / 6\end{array}$




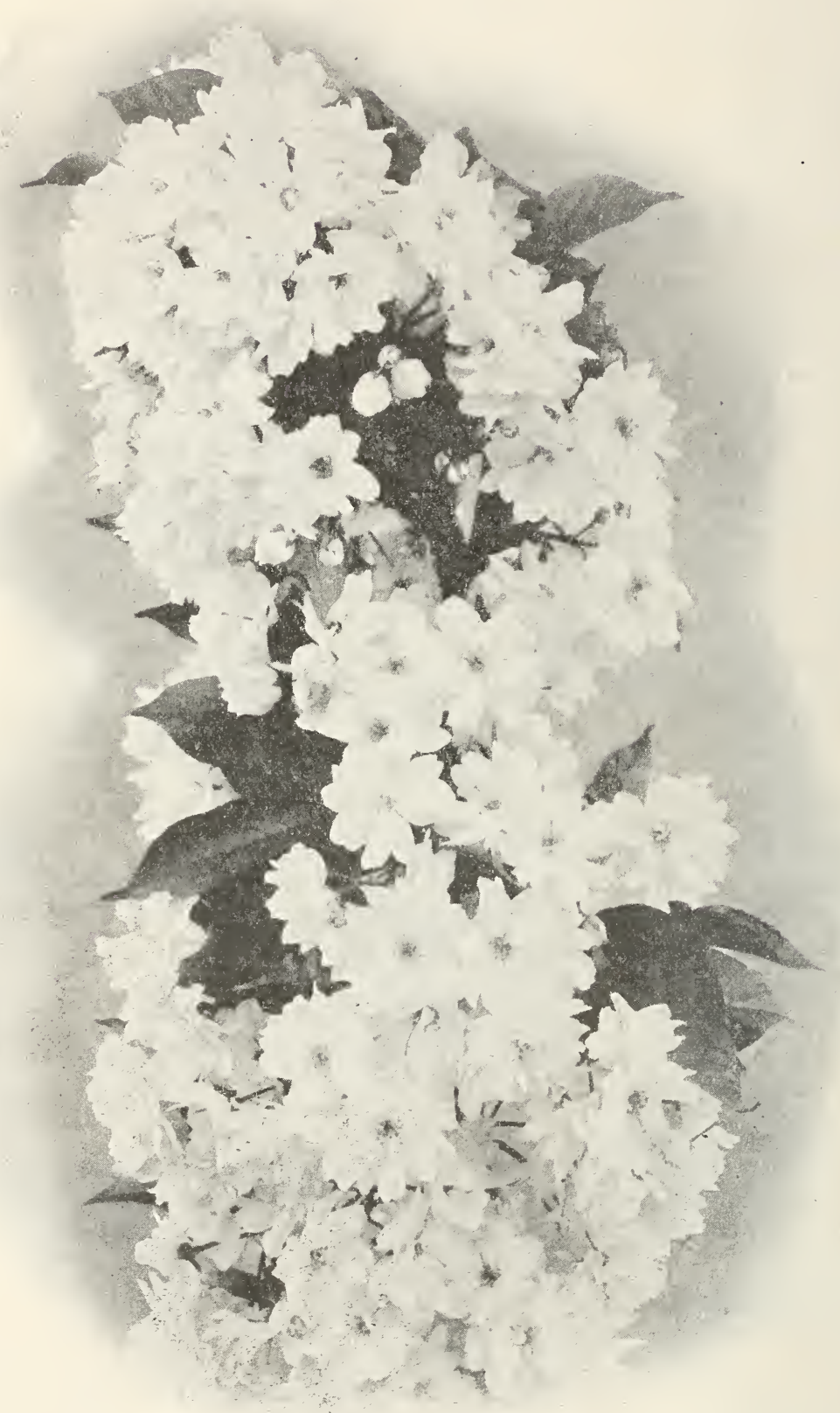

Cerasus serrulata.

The best double white Japanese Cherry.

Very free flowering (see page 59). 


\section{GENERAL COLLECTION (continued).}

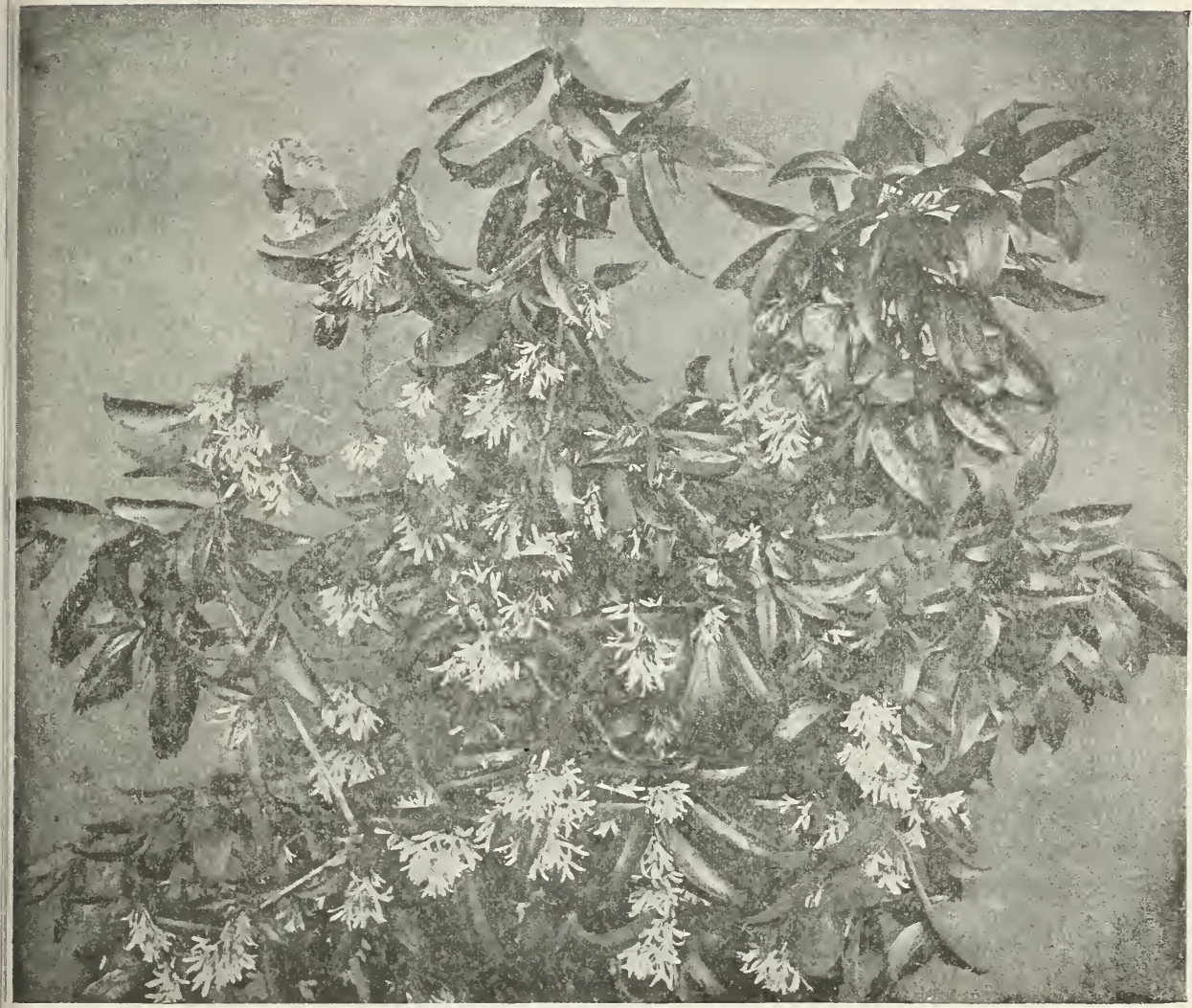

Chionanthus retusus.

During June, white flowers with strap shaped petals are produced in abundance (see page 59).

\section{Coronilla}

\section{Emerus}

\section{Corylópsis}

pauciflora

spicata

Córylus (Hazel Nut)

Avellana aurea

- laciniata

- pendula

- purpurea

- (purple leaved)

Colurna
Price per plant.

$\begin{array}{ll}- & 1 / 6 \\ - & 3 / 6 \\ - & 2 / 6 \\ & \\ - & 1 / 6 \\ - & 1 / 6\end{array}$

$3 / 6$ to $5 /$ -

$\begin{array}{ll}1 /- & 1 / 6 \\ 2 / 6 & 3 / 6\end{array}$

\section{Cotoneàster} acutifolia

This is the true species. The plant now in cultivation under this name is not true. A neat bushy deciduous shrub with dark glossy green ovate leaves. A good subject for covering walls

$1 / 6$

adpressa $\quad$ - 2/6

affinis - $\quad 1 / 6$

applanata (see page 45) $\quad$ 1/6

$\begin{array}{lll}\text { frigida } & 1 / 6 \quad 2 / 6\end{array}$

$\begin{array}{lll}\text { Nummularia } & 1 / 6 & 2 / 6\end{array}$

reflexa (See page 45) 1/6

Simonsii 1/- $1 / 6$

$\begin{array}{lll}\text { vulgaris (integerrima) } & \mathbf{1} / 6 & 2 / 6\end{array}$

See also Evergreen List page 36 
Crataegus (Hawthorn)

apiifolia

Carrierei (Lavellei)

chlorosarca

coccinea (scarlet-berried)

- corallina

Crus-galli (Cockspur)

- arbutifolia

- horrida

- prunifolia

- splendens

Douglasii

Korolkowii

melanocarpa

orientalis

- sanguinea

Oxyacantha (common)

- coccinea plena (Paul's

Double Scarlet)

- foliis argenteis

- multiplex (double white)

- pendula

- praecox (Glastonbury Thorn)

- punicea(scarlet-flowering)

pinnatifida major

punctata

- brevispina

tanacetifolia

and other varieties.

\section{Cydónia}

japonica (Pyrus), (Japanese Quince)

- atropurpurea

- cardinalis

- coccinea

- flore pleno

- Knap Hill Scarlet

- Moerloesii

- nivalis

- princeps

- rosea

Maulei

- alba

- superba

\section{Cytisus}

allous (multiflora)

(White Portugal Broom)

\section{Ardoinii}

Beanii

elongatus

flore alba

Kewensis

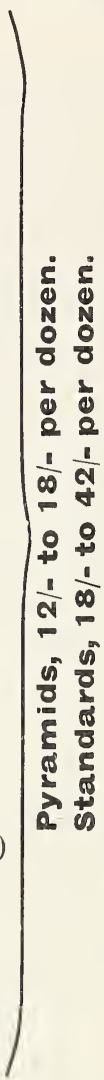

Cytisus (continued)

nigricans

purpureus

- incarnatus

- pendulus

schipkaensis

scoparius (Yellc

- Andreanus

- pallidus

- praecox

supinus

trifforus

\section{Dáphne}

\section{Davidia}

\section{Decaisnea}

Fargesii

\section{Desmodium}

nutans
Price per plant.

- $1 / 6$

$1 / 6$ to $2 / 6$

- $2 / 6$

$1 / 6 \quad 2 / 6$

$1 / 6 \quad 2 / 6$

$-/ 6 \quad 1 / 6$

/- $1 / 6$

- $1 / 6$

$1 / 6 \quad 2 / 6$

$1 / 62 / 6$

- $1 / 6$

Several of these Cytisus can be supplied as Standards, 3/6 to 7/6 each.

$\begin{array}{lcr}\text { Mezereum } & - & 1 / 6 \\ \text { - atro-rubrum } & - & 2 / 6 \\ \text { - flore albo } & 1 / 6 & 2 / 6\end{array}$

involucrata (see p. 46) 7/6 21/-

5/-

pendulifiorum $\quad 1 / 6 \quad 2 / 6$

tiliaefolium

$1 / 6-$

Dèutzia

corymbiflora $\quad$ - $1 / 6$

- erecta - $\quad 1 / 6$

crenata macrosepala- $\quad 1 / 6$ discolor major,

compact bush several feet high, well furnished with

ovate lanceolate leaves.

June the white flowers

are produced in the greatest profusion, much larger than the type, and borne in clusters along the whole length of the previous year's growth. The long arching sprays are of great value as cut flowers.

$1 / 6 \& 2 / 6$

gracilis

- hybrida rosea

$1 / 6$

kalmiaeflora (see

$\begin{array}{ll}- & 3 / 6 \\ 2 / 6 & 3 / 6 \\ 1 / 6 & 2 / 6 \\ 1 / 6 & 2 / 6 \\ 2 / 6 & 3 / 6\end{array}$

\begin{tabular}{lll}
\multicolumn{1}{c}{ illustration $p .63)$} & - & $1 / 6$ \\
Lemoinei & - & $1 / 6$ \\
- Boule de Neige & - & $1 / 6$ \\
parviflora & - & $1 / 6$
\end{tabular}




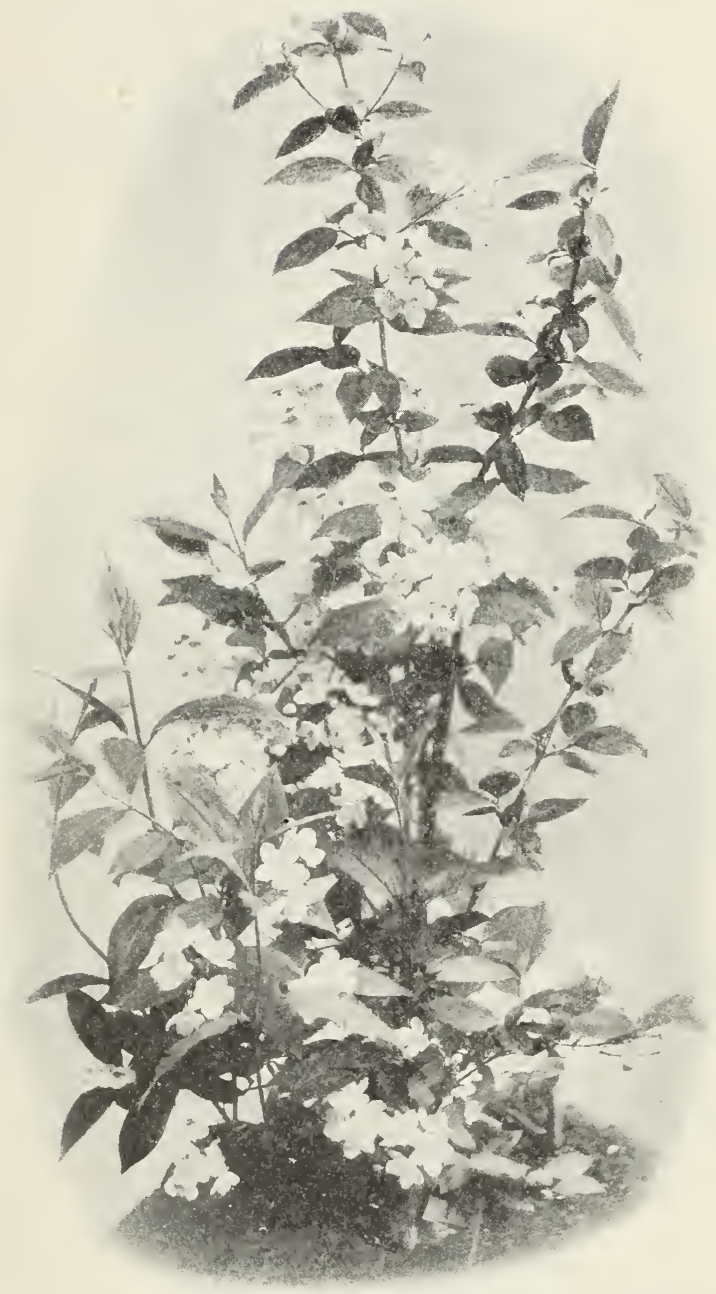

Deutzia kalmiafiora.

Flowers, rose-pink with white centre, 1/6 each.

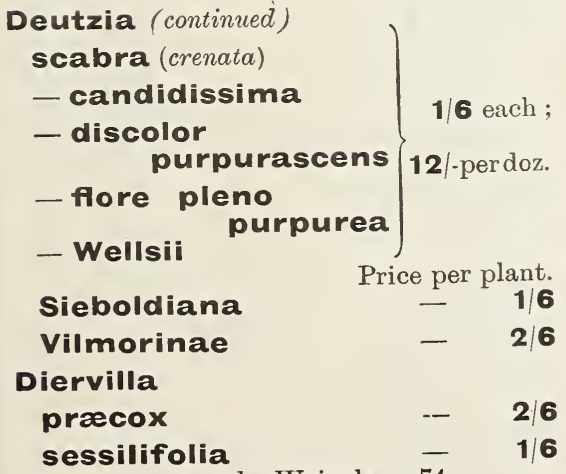

Dimorphanthus

See Aralia p. 5\%.

Diòspyros

Kaki

Lotus (Date Plum)

Prices

on

virginiana (Persimmon) application

Dirca Price per plant.

palustris (Leatherwood) - 2/6

Edgeworthia

papyrifera (chrysantha) - 2/6

Edwardsia (Sophora)

Macnabiana(microphylla) - 3/6 tetraptera (grandiflora) - 3/6 


\begin{tabular}{|c|c|c|}
\hline Elæàgnus & Price per & plant. \\
\hline argentea & - & $1 / 6$ \\
\hline Iongipes & - & $1 / 6$ \\
\hline umbellata & - & $1 / 6$ \\
\hline $\begin{array}{l}\text { Eucommia } \\
\text { ulmoides }\end{array}$ & $5 /-$ & to $\mathbf{7} / \mathbf{6}$ \\
\hline $\begin{array}{l}\text { Eucryphia } \\
\text { pinnatifolia (see } p a\end{array}$ & $g e 46)^{5 /-}$ & $10 / 6$ \\
\hline Euonymus & & \\
\hline americanus obov & atus - & $1 / 6$ \\
\hline amurensis (alatus) & - & $1 / 6$ \\
\hline atropurpureus & - & $1 / 6$ \\
\hline europaus (Spindle & ree) -16 & $1 / 6$ \\
\hline - augustifolius & $\mathbf{1} /-$ & $1 / 6$ \\
\hline - fructu-albo & - & $1 / 6$ \\
\hline latifolius & - & $1 / 6$ \\
\hline sachalinensis & - & $1 / 6$ \\
\hline verrucosus & - & $1 / 6$ \\
\hline Euptelea & & \\
\hline $\begin{array}{l}\text { Davidiana, A small } \\
10 \text { to } 20 \text { feet high, } \\
\text { neat orbicular leaves } \\
\text { assume fine colourin } \\
\text { the autumn }\end{array}$ & $\begin{array}{l}\text { tree } \\
\text { with } \\
\text { that } \\
\mathrm{g} \text { in }\end{array}$ & $2 / 6$ \\
\hline Exochórda & & \\
\hline Albertii & - & $1 / 6$ \\
\hline - macrantha & - & $1 / 6$ \\
\hline grandiflora & - & $1 / 6$ \\
\hline Fàgus (Beech) & & \\
\hline sylvatica (common) & $1 / 6$ & $2 / 6$ \\
\hline - asplenifolia & $2 / 6$ & $3 / 6$ \\
\hline - cristata & $2 / 6$ & $3 / 6$ \\
\hline - - argenteis & $3 / 6$ & $5 /-$ \\
\hline - pendula & $3 / 6$ & $7 / 6$ \\
\hline - purpurea (Purpl & ech) & $7 / 6$ \\
\hline - - pendula & $5 /-$ & $10 / 6$ \\
\hline quercifolia & $2 / 6$ & $3 / 6$ \\
\hline - tricolor & $3 / 6$ & $\mathbf{5} /-$ \\
\hline - Zlatia & - & $2 / 6$ \\
\hline Forsythia & & \\
\hline intermedia & & $1 / 6$ \\
\hline suspensa & per doz. & $1 / 6$ \\
\hline viridissima & $12 /-$ & $1 / 6$ \\
\hline - variegata & & $1 / 6$ \\
\hline
\end{tabular}

Fràxinus (Ash)

americana pensylvanica - $2 / 6$

bracteata (see page 46) - 5/dimorpha
Fraxinus (continued)

Price per plant.

excelsior (common)

$\mathbf{1} /$ - to $2 / 6$

- arbutifolia

$2 / 6$

- argenteo-variegata -

26

- aucubaefolia

$1 / 6$ to $2 / 6$

- pendula

$8 / 6 \quad 10 / 6$

Mariesii

$3 / 65$ -

Ornus (Ornus europua) (Flowering Ash) 2/6 5/-

viridis (Juglandifolia)

$2 / 6$

Fremóntia

californica

$7 / 6 \quad 106$

Fuchsia

americana elegans

Corallina

globosa

Mdme. Corneilson

microphylla

Riccartonii

Thomsonii

$$
\begin{aligned}
& 1 / \cdot \\
& \text { per doz } 1 / \text { - } \\
& \text { per doz. } 1 / \text {. } \\
& 9 / \quad 1 / \text {. } \\
& 1 /- \\
& 1 / \text { - }
\end{aligned}
$$

\section{Genista}

æethnensis

$-\quad 16$

capitata

daurica

hispanica

pilosa

prostrata

purgans

radiata

sagittalis

tinctoria

- flore pleno

- mantica

virgata

- $1 / 6$

$1 /-\quad 1 / 6$

$1 /-\quad 1 / 6$

$1 /-\quad 1 / 6$

$1 /-\quad 1 / 6$

- $1 / 6$

- $1 / 6$

$1 /-\quad 1 / 6$

$1 /-\quad 1 / 6$

- $1 / 6$

$1 / 6 \quad 2 / 6$

- $1 / 6$

Ginkgo (Maiden Hair Tree)

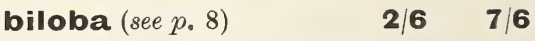

Gleditschia

sinensis (horrida) $\quad 2 / 6 \quad 3 / 6$

- pendula $\quad 5 /-\quad 7 / 6$

$\begin{array}{lll}\text { triacanthos } & 1 / 6 & 2 / 6\end{array}$

- excelsa pendula $2 / 6 \quad 3 / 6$

Gymnocladus

canadensis

Halèsia (Snowdrop tree)

hispida (Pterostyrax) - $2 / 6$

$\begin{array}{lll}\text { tetraptera } & 1 / 6 & 2 / 6\end{array}$

Halimodèndron (Salt tree)

argenteum $\quad 1 / 6$ 
Hamamèlis arborea japonica

Zuccariniana $2 / 6 \quad 3 / 6$ mollis (see page 46)

$3 / 65 /$.

virginica (Witch Hazel) $1 / 6 \quad 2 / 6$

Hedysärum

multijugum

Hibiscus

syriacus (Althcea frutex)

- variegatus

very fine single and

double varieties by name

Standards in variety, our selection

Price per plant.

$$
3 / 6 \text { to } 5 \text { - }
$$

Idèsia

polycarpa

Price per plant.

Indig'ofera

decora alba $\quad-\quad 2 / 6$

Gerardiana (floribunda) $1 / 6 \quad 2 / 6$

Jamésia

americana $\quad-\quad 2 / 6$

Jùglans

Cathayensis (see p.47) - 10/6

nigra (Black Walnut) 2/6 5/-

regia (common Walnut) $1 / 6 \quad 3 / 6$

- laciniata

$3 / 6 \quad 5 /$

Kèrria (Corchorus)

japonica

- fore pleno

- variegata

$1 /-\quad 1 / 6$

$1 /-\quad 1 / 6$

$1 /-\quad 1 / 6$

Koelreutèria

paniculata

$1 / 6 \quad 3 / 6$

Hortensia

$1 / 6 \quad 5 /$

- variegata

$1 / 6 \quad 2 / 6$

- mandschurica

$1 / 6 \quad 5 /-$

- Mariesii

$1 / 6 \quad 5$

- Otaksa

$1 / 6 \quad 2 / 6$

- rosea

$2 / 6 \quad 3 / 6$

- Thomas Hogg

$1 / 6 \quad 2 / 6$

- Veitchii, sterile white

flowers, 3 inches in

diameter, confined to

the outer circumference

of the inflorescence. $\quad-\quad 3 / 6$

paniculata

quercifolia grandiflora $1 /-\quad \begin{array}{r}1 / 6 \\ -\quad 2 / 6\end{array}$

The blue flowers which are produced by some Hydrangeas are not normal, but are due to the action of certain chemical constituents which the plants derive from the water or soil.

Hypèricum

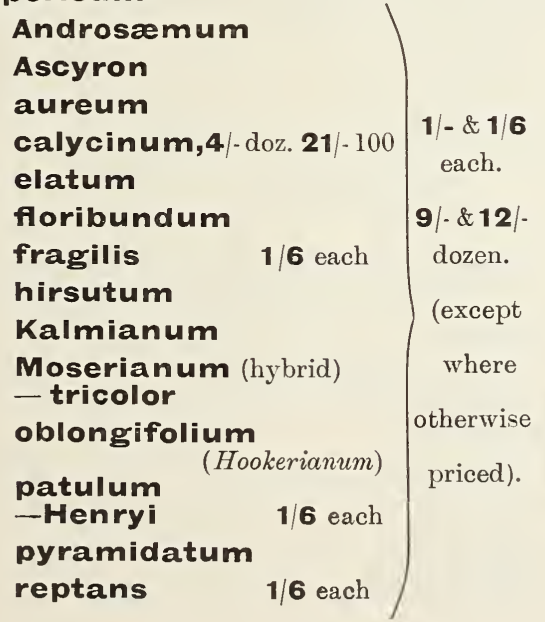

Labùrnum

Adamii

$\begin{array}{lll}\text { (vulgare purpurascens) } & 1 / 6 & 2 / 6 \\ \text { lpinum (Scotch) } & 1 / 6 & 2 / 6 \\ \text { ulgare (common) } & 1 / 6 & 2 / 6 \\ \text { Alschingeri } & - & 2 / 6 \\ \text { aureum } & - & 2 / 6 \\ \text { autumnale } & - & 2 / 6 \\ \text { monstrosum } & - & 2 / 6 \\ \text { - cristatum } & - & 2 / 6 \\ \text { Parksii } & - & 2 / 6 \\ \text { pendulum } & - & 2 / 6 \\ \text { quercifolium } & - & 2 / 6 \\ \text { Vossii } & - & 2 / 6\end{array}$

Laùrus

Benzoin (Lindera Benzoin) - $\quad$ 2/6

Leycesteria

formosa

- variegata

$1 /-\quad 1 / 6$

$-\quad 1 / 6$

Lespedèza

bicolor

$1 / 6 \quad 2 / 6$

Lindera

sericea

$3 / 6$

triloba

- $3 / 6$

$-\quad 3 / 6$

styraciflua $\quad 2 / 6 \quad 5 /$ -

Liriodèndron (Tulip Tree)
tulipifera
$2 / 6 \quad 5 /-$
- aurea
$3 / 6 \quad 5 /$

præcox

\section{Liquidambar}




\section{GENERAL COLLECTION (continued)}

Lonicèra

(Shrubby Honeysuckle)

Each Perdoz.

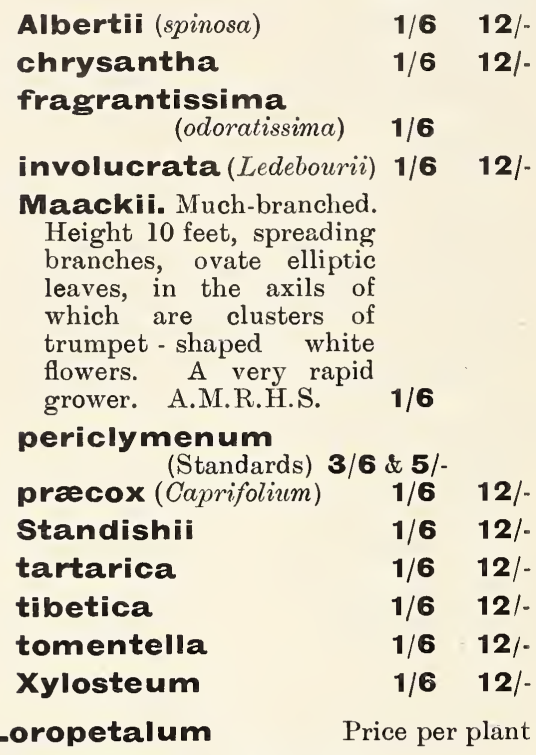

chinense. A winter-

flowering shrub. Pure

white flowers, with long

strap-shaped petals re-

sembling the Chionanthus $2 / 6$ to $\mathbf{3} / \mathbf{6}$

\section{Lycium}

europeum

- variegatum

- 1/-

Maclùra

aurantiaca

$$
\text { (Osage Orange) - 1/6 }
$$

Magnôlia

acuminata

conspicua (Yulan)

* Soulangeana

- - nigra

\section{glauca}

- Thomsoniana

$2 / 6$ to $5 /-$

$5 /-10 / 6$

$2 / 6 \quad 10 / 6$

- $7 / 6$

$5 /-\quad 7 / 6$

$5 /-\quad 7 / 6$

hypoleuca. Leaves obo-

vate, bright green above, glaucous beneath, 13 to 15 or more inches long and 7 to 8 inches broad; flowers 6 to 7 inches across with creamy white petals and red purple anthers; freely produced on adult trees's

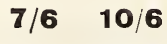

Kobus

- 10/6

* Lennei

(see illustration p. 67) $\mathbf{5 / -} \quad \mathbf{1 0 / 6}$

parvifiora (see p. 48) $\quad \mathbf{7 / 6} \quad \mathbf{1 0 / 6}$

purpurea (obovata)
Magnôlia (continued)

(10/6

salicifolia. Slender

branches clothed with

light green willow-like

leaves 5 to 6 inches long,

silvery white on the under

surface

$-\quad 10 / 6$

stellata (Halleana) $\quad 3 / 6 \quad 10 / 6$

- rosea

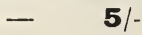

tripetala (Umbrella) $\mathbf{2} / \mathbf{6} \quad \mathbf{5} /$ -

$\begin{array}{lll}\text { Watsoni (see p. 48) } \quad \mathbf{7} / \mathbf{6} & \mathbf{1 0} / \mathbf{6}\end{array}$

Of the varieties marked

thus * we hold a stock of exceptionally fine specimens. Particulars and prices on application.

\section{Morus}

alba $2 / 6$ to $3 / 6$

- pendula $5 /-\quad 7 / 6$

nigra (common Mulberry) 5/- 21/.

Myrica

cerifera

(Candleberry Myrtle) — $\quad 1 / 6$

Gale (Sweet Gale) 1/- $1 / 6$

\section{Negundo}

californicum

fraxinifolium

aureum $1 / 6 \quad 3 / 6$

(aceroides) 1/- $\quad 1 / 6$

- albo-variegatum $1 / 6 \quad 5 /-$

- aureo-variegatum 1/6 5/-

- marginata

elegans $2 / 6 \quad 5 /$.

Neillia. See Spiræea page 72

Neviusia

alabamensis $\quad-\quad 2 / 6$

Nuttàllia

cerasiformis $\quad-\quad 1 / 6$

Ononis

fruticosa $\quad-\quad 2 / 6$

\section{Ornus}

europaea (Fraxinus ornus)

(Flowering Ash) 2/6 5/.

mariesii $3 / 6 \quad 5 /=$

Paónia Moutan (Tree

Pæony) best named varieties $\mathbf{3} / \mathbf{6}$ to $\mathbf{7} / \mathbf{6}$

Paliurus (Christ's Thorn)

aculeatus (australis) _ $\quad$ 1/6

Parrótia

Jacquemontii _ $3 / 6$

persica

$1 / 6$ to $5 /$ -

Paulównia

imperialis $\quad 2 / 6 \quad 5 /-$ 


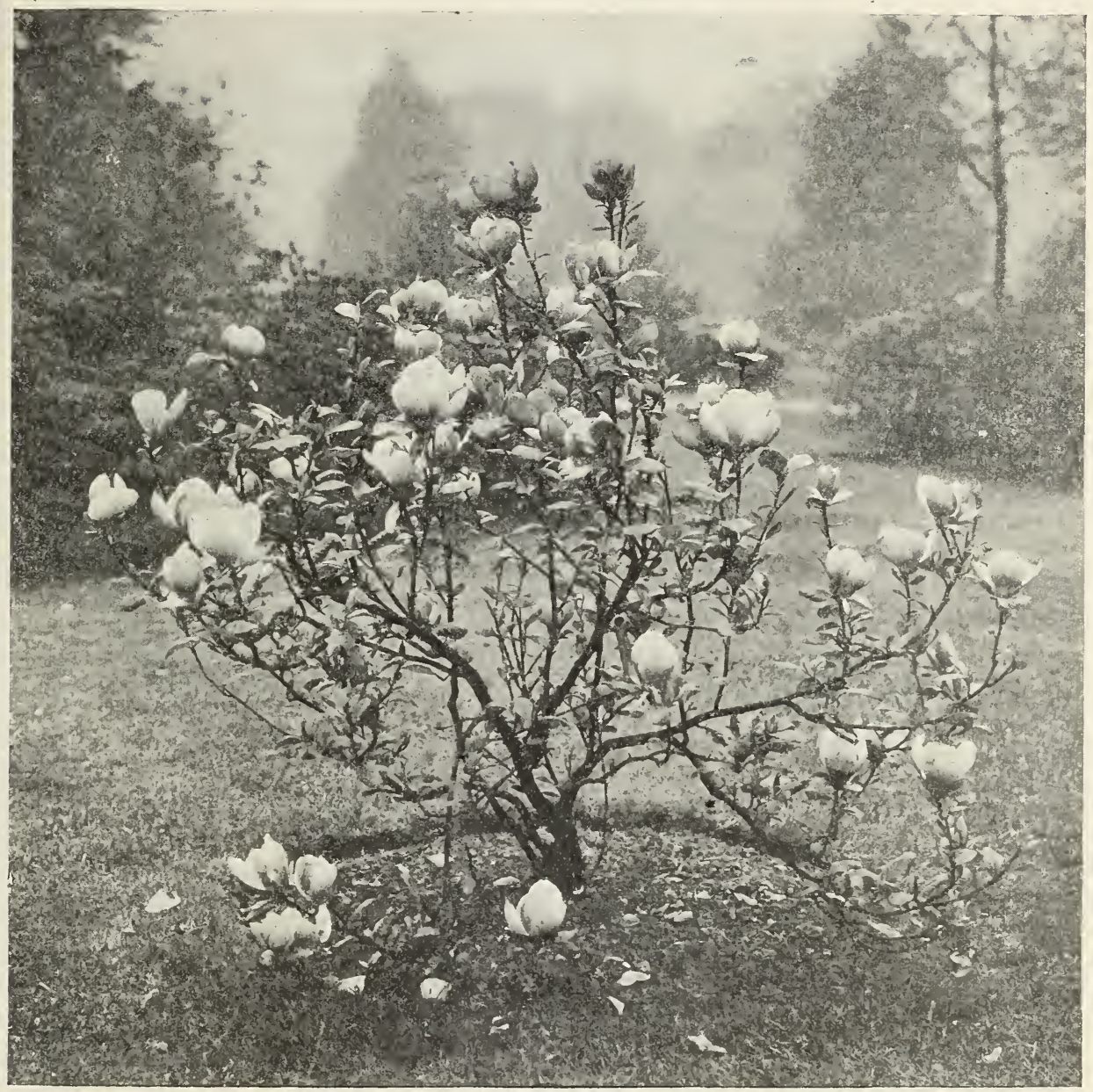

Magnolia Lennei.

A very free-flowering Magnolia with large flowers that are reddish-purple on the outside and white on the inside.

\section{See page 66.}

Pavia, see Aesculus page 56

\section{Perowskia Price per plant.} atriplicifolia

A beautiful shrub bearing long spikes of blue flowers in the autumn.

Philadelphus (Mock Orange) coronarius (sweet)

- argenteovariegatus

- erectus

- Falconeri

- flore pleno

Gordonianus

grandiflorus

- speciosissimus
1/- \&

$1 / 6$

each;

9/ 8

12 -

per

doz.

Price per plant.

Philadèlphus (continued)

$\begin{array}{lrr}\text { Lemoinei } & 1 /-\& & 1 / 6 \\ \text { - Avalanche } & - & 1 / 6\end{array}$

Very large white fragrant flowers.

- Bannière - 1/6

- Boule d'Argent $\quad$ - 1/6

- Bouquet Blanc _ $\quad 1 / 6$

- Conquete - $1 / 6$

- Fantaisie (see page 49) - $\quad 1 / 6$

- Gerbe de Neige _ 1/6

- Candelabre _ 1/6

- Manteau d'Hermine - 1/6

- Mer de glace _ $1 / 6$

- Mont Blanc _ $\quad 1 / 6$ 
Philadelphus (continued).

Price per plant.

Lemoinei

- Pavillon Blanc duced in clusters.

- purpureo-maculatus (see page 49)

- Rosace (see page 49). — $\quad$ 1/6

- Virginal (see page 49) — $\quad \mathbf{2 / 6}$

mexicanus - 1/-

microphyllus $\quad-\quad 1 / 6$

Satsumi $\quad-\quad 1 / 6$

trifiorus $\quad-\quad 1 / 6$

Voie lactée $\quad$ - $\quad 1 / 6$

Photinia

villosa (variabilis) $\quad$ 2/6

Plànera. See Zelkova, p. 74

Plàtanus

acerifolia (London Plane) 2/6 $10 / 6$

- süttneri - $3 / 6$

orientalis (Oriental Plane) $2 / 6$ to $10 / 6$

Populus (Poplar)

alba (Abele)

$1 / 6$ to $2 / 6$

- argentea (nivex) $\quad 1 / 6 \quad 3 / 6$

- Bolleana (pyramidalis) $1 / 6$

balsamifera (Balsam Poplar or Tachamahac) $\quad 1 / 6$

- Iaurifolia (laurifolia) $\quad$ 1/6

canescens pendula -

fastigiata (nigra pyramidalis)

$1 / 6$

Iasiocarpa (see page 48) -

monilifera (deltoidea) $\quad 1 / 6$

- canadensis aurea $2 / 6$

- nova

$1 / 6$

tremula (Aspen)

$1 / 6$

- pendula

$2 / 6$

$5 /-$

$3 / 6$

$3 / 6$

$3 / 6$

$3 / 6$

$10 / 6$

$3 / 6$

$3 / 6$

$3 / 6$

2/6

- pendula

$5 /-$

Potentilla

Friedrichsenii

fruticosa

Salesoviana

$-\quad 1 / 6$

Veitchii (see page 52 )

$-\quad 1 / 6$

- $1 / 6$

$3 / 6$

\section{Prúnus}

Besseyi

$1 / 6 \quad 2 / 6$

Mume, double white _ $\quad 3 / 6$

一 double pink $\quad$ - 3/6

Myrobalana f. pl. (cerasifera) $1 / 6$

Pissardii

(cerasifera atropurpurea) $1 / 6 \quad 5 /$ -

- Moseri fi. pl. _ 2/6

Purple foliage, double rose-

coloured flowers. A.M. R.H.S.
Prúnus (continued)

Price per plant.

sinensis flore alba pleno

$1 / 6$ to $3 / 6$

- - rosea pleno $\quad 1 / 6 \quad 3 / 6$

spinosa flore pleno

(double-flowering Sloe) — $\quad 1 / 6$

- purpurea

$1 / 6$

tomentosa

$1 / 6$

triloba (Amygdalopsis) $\quad 1 / 6 \quad 2 / 6$

Ptèlea

trifoliata (Hop Tree) $\quad-\quad 1 / 6$

- aurea $\quad$ - 1/6

Pterocàrya

$\begin{array}{lll}\text { caucasica (fraxinifolia) } & 1 / 6 & 3 / 6\end{array}$

stenoptera (lavigata) $\quad 1 / 6 \quad 3 / 6$

\section{Pyrus}

Pear and Apple Section.

coronaria

1/6 2/6

- ก. pl.

$2 / 6 \quad 3 / 6$

foribunda

$1 / 6 \quad 3 / 6$

- atro-sanguinea $\quad 1 / 6 \quad 3 / 6$

Malus (Dartmouth Crab) 2/6 3/6

- alba plena $\quad 2 / 6 \quad 3 / 6$

$\begin{array}{lll}\text { - Bertinii } & 2 / 6 & 3 / 6\end{array}$

- Elise Rathke (pendulous) $3 / 6$

- Halleana $\quad 1 / 6 \quad 3 / 6$

- Neidzwetzkyana $1 / 6 \quad 3 / 6$

prunifolia (Siberian Crab) 1/6 2/6

$\begin{array}{lll}\text { salicifolia } & 1 / 6 & 2 / 6\end{array}$

- pendula $\quad 2 / 6 \quad 3 / 6$

$\begin{array}{lll}\text { Scheideckeri } & 1 / 6 & 2 / 6\end{array}$

(See illustration p. 69)

spectabilis - 2/6

- fiore pleno - 2/6

See also Fruit Tree Catalogue, free on application.

White Beum and Service Section.

\section{Pyrus}

Aria (White Beam Tree) $\quad 1 / 6$ to $2 / 6$

$\begin{array}{lll}\text { - chrysophylla } & 2 / 6 & 3 / 6\end{array}$

Aucuparia (Mountain Ash)-/6 3/6

- pendula $3 / 6 \quad 5 /-$

- Moravica laciniata - 2/6

$\begin{array}{lll}\text { intermedia } & 1 / 6 & 2 / 6\end{array}$

lutescens $\quad 2 / 6 \quad 5 /-$

pinnatifida (fennica) $\quad 1 / 6 \quad 2 / 6$

Sorbus (True Service) $\quad 1 / 6 \quad 3 / 6$

- foliolosa - $2 / 6$

Torminalis $\quad 1 / 6 \quad 2 / 6$

$\begin{array}{lll}\text { vestita } & 2 / 6 & 3 / 6\end{array}$




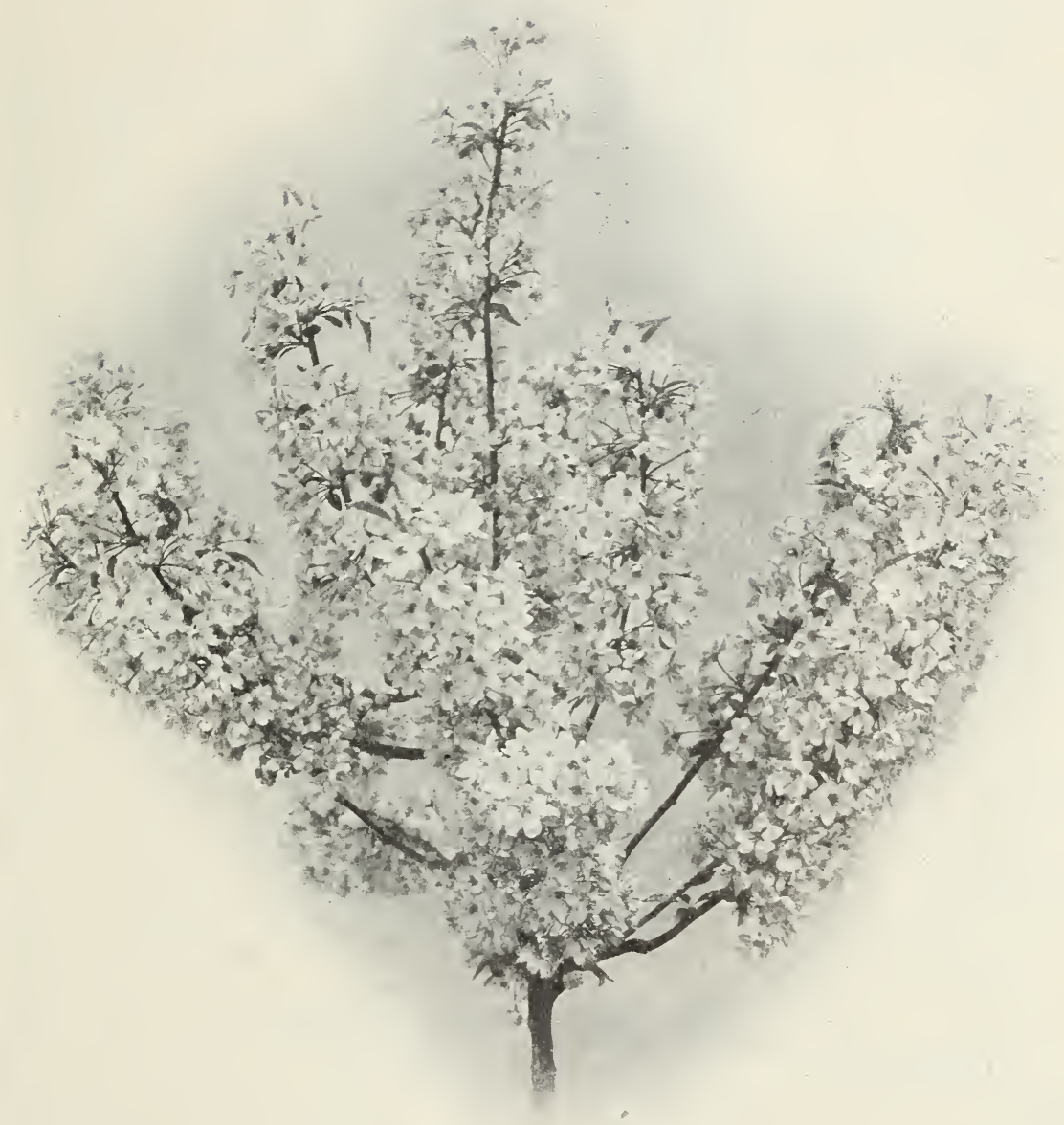

Pyrus Scheideckeri.

A most beautiful free-flowering Crab Apple. Flowers blush-pink changing to white. Opens early in May (see page 68).

Quercus (Oak)

alba (see $p .41)$

aquatica (nigra)

Banisteri (ilicifolia) (see p. 41)

Cerris (Turkey Oak)

Price per plant

$$
\begin{aligned}
& \text { - } \quad 3 / 6 \\
& 2 / 6 \text { to } 3 / 6 \\
& \text { - } 36
\end{aligned}
$$

- argentea variegata $3 / 6 \quad 5 /-$ coccinea (Scarlet Oak),

$\begin{array}{lll}\text { Waterer's var. (see p. 4l) } & \mathbf{3} / 6 & \mathbf{1 0} / 6\end{array}$ conferta (pannonica) (see p. 41)

dentata (Daimyo)

$3 / 6 \quad 10 / 6$ falcata (Spanish Oak) (cuneata) $\quad \mathbf{3 / 6}$

Louettii (sessiliflora mespilifolia)

Price per plant.

Quercus (Oak) (continued) macrocarpa (Burr Oak) - 3/6 Marylandica - $\quad \mathbf{5} /-$ mongolica $\quad-\quad 3 / 6$ nigra (see page 41) - 3/6 palustris (Marsh Oak), (see $p_{2}$ 4l) $3 / 6$ pedunculata (English

Oak) $1 / 6 \quad 5 /-$ - argenteo-variegata $2 / 6 \quad 3 / 6$ - Concordia $2 / 6 \quad 5 /$ - fastigiata $3 / 6 \quad 5 /-$

- pectinata asplenifolia $8 / 6 \quad 5 /$ 


\begin{tabular}{|c|c|c|}
\hline \multicolumn{3}{|c|}{$\begin{array}{l}\text { Quercus (continued). Price per plant. } \\
\text { pedunculata }\end{array}$} \\
\hline purpurascens & $2 / 6$ to & \\
\hline Phellos (Willow Oak) & - & \\
\hline rubra & $2 / 6$ & \\
\hline - aurea & $3 / 6$ & \\
\hline Tauzin $\left(T_{o z a}\right)$ & $3 / 6$ & \\
\hline \multicolumn{2}{|c|}{ tinctoria (velutina)(see p. 41)- } & \\
\hline Zang (Mirbeckii) & 26 & \\
\hline
\end{tabular}

For Select List of Evergreen and Deciduous Rhàmnus

Oaks (see p. 41).

\section{alpina \\ Frangula

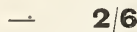 \\ Imeretinus \\ $1 /-$ to $1 / 6$ \\ $1 / 6 \quad 2 / 6$ \\ See also Evergreen List, page 39.}

\section{Rhodotypos}

kerrioides

Rhus (Sumach)

copallina

Cotinus (Venetian)

- purpurea

cotinoides

glabra

- laciniata

Osbeckii

Toxicodendron

(Poison Oak) (Poison Ivy) - $\quad \mathbf{1 / 6}$

- radicans (Ampelopsis

$$
\begin{aligned}
& \text { typhina } \\
& \text { japonica) 1/6 } \\
& \text { - laciniata }
\end{aligned}
$$$$
-\quad 2
$$$$
-\quad 1 / 6
$$$$
\text { - 1/6 }
$$$$
\text { - } 3 / 6
$$$$
\text { - 1/6 }
$$$$
1 / 6 \quad 2 / 6
$$$$
1 / 62 / 6
$$

Ribes

aureum

chilinus

$2 / 6$

$2 / 6$

$2 / 6$

Gordonian

missouriense (americanum) prostratum

sanguineum

- albidum

- atrosanguineum

- - fi. pl.

- glutinosum

- King Edward VII

$1 /-\quad 1 / 6$

$1 /-\quad 1 / 6$

$1 /-\quad 1 / 6$

speciosum

$1 /-\&$

$1 / 6$

each ;

9/- \&

$12 /-$

per

doz.

Robinia

hispida (Rose Acacia) $\quad 1 / 6$ to $2 / 6$

$\begin{array}{lll}\text { - macrophylla } & 1 / 6 & 2 / 6\end{array}$

Kelseyi - $2 / 6$

neo-mexicana $\quad-\quad 2 / 6$

- Standards - $\mathbf{3 / 6}$

Pseud-acacia (Locust Tree) $1 / 6$
Robinia (continued).

Price per plant

Pseud-acacia

- angustifolia

elegans $1 / 6$ to $3 / 6$

- aurea

$2 / 6 \quad 3 / 6$

- Bessoniana

$1 / 65 /-$

- Decaisneana $\quad 2 / 6 \quad 3 / 6$

- inermis, Standards $3 / 6 \quad 5 /-$

- - foliis variegatis $2 / 6 \quad 3 / 6$

$\begin{array}{lll}\text { - monophylla } & 1 / 6 & 2 / 6\end{array}$

- Rozynskiana _ _ $2 / 6$

$\begin{array}{lll}\text { - semperflorens } & 1 / 6 & 2 / 6\end{array}$

Rubus (Bramble)

australis

$1 / 6$ to $2 / 6$

bambusarum (see p. 50). $2 / 6 \quad 3 / 6$

biflorus $\quad-\quad 2 / 6$

chroosepalus (see p. 49) $\quad \mathbf{2 / 6}$

deliciosus - $2 / 6$

Hagelliformis (see p. 50) $2 / 6 \quad 3 / 6$

fruticosus (common) - $\quad-/ 6$

- fiore albo pleno : $-1 /$ -

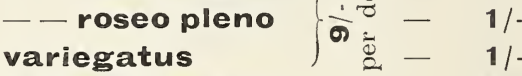

innominatus $(\sec p .50) \quad \mathbf{1 / 6}$

laciniatus - $1 /-$

lasiostylus. The white

stems of this Rubus are very

striking (see illus. page 71). - $\quad$ 1/6

Lawton's - 1/.

leucodermis $\quad-\quad 1 / 6$

nutkanus - $\quad 1 / 6$

odoratus - 1 -

phonicolasius - 1/-

sorbifolius $\quad-\quad 1 / 6$

spectabilis - $1 /$ -

Veitchii (see page 50) $\quad 5 /$.

Wilson, Junior, per doz., 9/- ; 1/-

Salisburia (Maiden-hair Tree).

See Ginkgo biloba, page 8

Sàlix (Willow)

alba $-/ 6$ to $2 / 6$

* - vitellina (Golden Willow) -/6

$\begin{array}{lll}\text { americana pendula } & 2 / 6 \quad 3 / 6\end{array}$

babylonica (Weeping

$\begin{array}{lll}\text { Willow) } & \text { 1/6 } & \text { 2/6 }\end{array}$

- annularis (Ringlet

*Britzensis $\quad-/ 6 \quad 2 / 6$

Caprea pendula (Kil-
marnock Weeping Willow) 2/6 3/6

* cardinalis (Scarlet

Willow) -/6 1/6




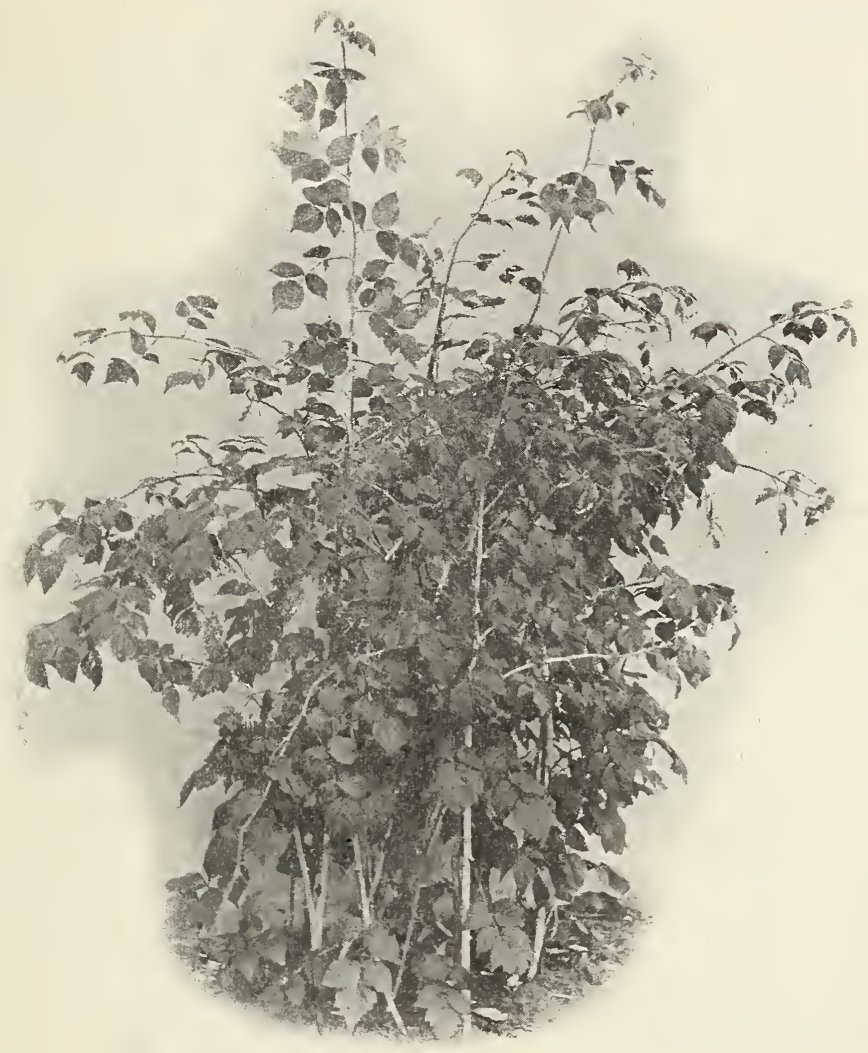

Rubus lasiostylus (see page 70).

Price per plant.

Sàlix (Willow) continued

\begin{tabular}{|c|c|c|}
\hline Iucida & - & \\
\hline purpurea (Purple Willow) & & \\
\hline is (Silver Willow) & $1 /-$ & \\
\hline $\begin{array}{l}\text { rinifolia } \\
\text { a pendula } \\
\text { (S. repens argentea) }\end{array}$ & -16 & \\
\hline
\end{tabular}

Those marked thus $\left(^{*}\right)$ are very fine for massing.

Special prices for quantities, on application.

\section{Sambucus (Elder)}

canadensis, height 6 to 12 feet; huge cymes of creamwhite flowers, bold graceful habit; purplish-black fruits sparingly produced, but the flower-stalks persist and assume a purplish hue. Suitable for borders of lakes or streams sambucus nigra

- argenteo-variegata

- aurea

- fructu albo

- laciniata

- pulverulenta

- pyramidalis

racemosa

- serratifolia

- - foliis aureis $\quad-\quad 1 / 6$

Shepherdia Price per plant.

argentea $\quad-\quad 2 / 6$

Sophora

$\begin{array}{lcc}\text { japonica } & 1 / 6 \text { to } & 2 / 6 \\ - \text { pendula } & 5 /- & 7 / 6 \\ \text { viciifolia } & - & 2 / 6\end{array}$

(See also Edwardsia p. 63)

1/- \& each

$12 /-$

doz.

junceum (Spanish Broom) -/9 1/.

\section{Spiræa}

Aitchisonii $\quad-\quad 1 / 6$

Anthony Waterer $\quad-\quad 1 /$. 


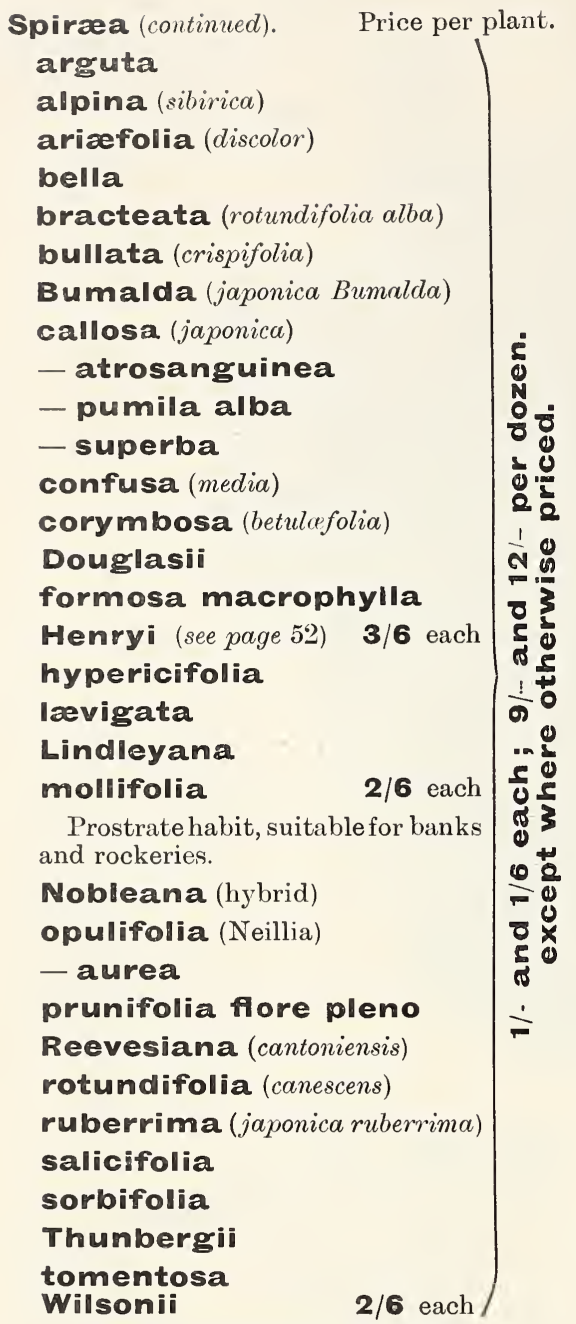

A new species from $W$. Hupeh, producing corymbs of white flowers borne on long arching growths during June and July.

For herbaceous Spiræas, see Herbaceous

Plant Catalogue, free on application.

\section{Stachyurus}

japonica (præcox)

Staphylea (Bladder Nut)

$\begin{array}{lcc}\text { Bumalda } & 1 / 6 \text { to } & 2 / 6 \\ \text { colchica } & 1 / 6 & 2 / 6 \\ \text { pinnata } & - & 1 / 6\end{array}$

Stephanàndra

filexuosa

Tanakx

Stuàrtia

pentagyna
Styrax

Price per plant.

japonicum

$2 / 6$

Rather dense habit, from 8 to 12 feet high, but ocasionally a low tree 20 to 25 feet high, with deep green ovate-lanceolate leaves. Flowers profusely in early summer; pure white fragrant blossoms. F.C.C. R.H.S.

\section{Obassia $5 /-10 / 6$}

One of the finest of the small profuse flowering trees clothed during the summer months with imposing foliage, bright lustrous green above and downy beneath. The flowers are produced in profusion after the plant has become established, upon terminal racemes, 6 to 8 inches long, each with from 15 to 30 pure white campanulate flowers, strongly but pleasantly fragrant. F.C.C. R.H.S.

\section{Symphoricàrpus}

\section{occidentalis}

The berries on this species are very

large and produced in abundance racemosus (Snowberry) ) 9d. \& 1/- purpureus vulgaris variegatus each ; 6/- \& 9/doz.

\section{Symplocos}

cratagoides

Syringa (Lilac)

Species.

Emodii, white

- variegata, white,

foliage edged gold $\quad-\quad 1 / 6$

japonica, white _ $\quad 1 / 6$

Josikæa, dark lilac _ $\quad$ 1/6

pekinensis, white _ $\quad 1 / 6$

persica, pale lilac _ $\quad 1 / 6$

- alba, white $\quad$ - 1/6

villosa, rosy pink _ $\quad 1 / 6$

vulgaris (Common Lilac) $1 /$ - to $1 / 6$

Syringa vulgaris, single varieties.

alba, white 1/-

$1 / 6$

- grandiflora, white,

\section{Aline Marqueris,}

$$
\text { very fine - 1/6 }
$$

reddish purple $\quad-\quad 1 / 6$

Charles $X_{\text {, }}$, rosy purple $\quad$ 1/6

Congo, very large, red, fine - 2/6

Dr. Lindley, rosy purple - $1 / 6$

Frau Bertha Dammann,

Gént des Batailles,

$$
\text { white }-1 / 6
$$

La Ville de Troyés,

$$
\text { dark red - } 1 / 6
$$

purplish red - $1 / 6$

Lucie Baltet, flesh colour - 1/6

Marie Legraye, white - 1/6 


\section{GENERAL COLLECTION (continued)}

Syringa (continued). $\quad$ Price per plant.

Madame Francisque Morel, violet-pink $1 / 6$

Madame Kreuter, bright rose - 1/6

Mdlle. Melide Laurent, ashy lilac - 2/6

Noisettlana, white $\quad-\quad 1 / 6$

Philemon, dark red $\quad-\quad 1 / 6$

Princess Marie, pinkish lilac - $2 / 6$

Souvenir de L. Späth, dark red

Toussaint Louvertre, dark crimson, changing to dark violet and others.

Syringa vulgaris, double varieties.

Alphonse Lavallee,

bright blue, violet shade -

Charles Joly, very dark red -

Condorcet, large spikes, blue, white beneath - $2 / 6$

Doyen Keteleer, pale rose, large spike - 2/6

Dr. Masters, clear lilac _ $1 / 6$

Emile Lemoine, flesh-rose - $\quad$ 1/6

Grand duc Constantin, ashy lilac - $1 / 6$

La Tour d'Auvergne, violet-purple - $1 / 6$

Lemoinei, rose, turning to lilac $-\mathbf{1 / 6}$

Léon Simon, blue, buds coral - 1/6

Linne, reddish lilac $\quad-\quad 2 / 6$

Louis Henry, violet-rose - 1/6

Madame Abel Chatenay, milk white - 2/6

- Casimir Perier, creamy white - $1 / 6$

- Lemoine, pure white,

Marc Micheli, clear lilac-blue - 1/6

Marechal de Bassom-pierre, pinkish lilac - 2/6

Mathieu de Dombasle, mauve, shaded rose - $1 / 6$

Michael Buchner, pale lilac. fine - 1/6

Monsieur Lepage, ashy lilac - 2/6

President Carnot, pale lilac, white centre - 2/6

President Grevy, rosy lilac - 1/6

- Loubet, light purple -

- Viger, bluish lilac
Syringa (continued).

Price per plant.

Renoncule, pale mauve $\quad-\quad 1 / 6$

Virginité, pale blush - $\quad 1 / 6$

William Robinson, violet

$$
\text { and others. }
$$

$$
\text { mauve - 2/6 }
$$

We shall be pleased to supply a collection of Lilacs (our selection), at 12/-, 18/- and 24/- per dozen.

All the above are on their own roots.

\section{Standards in variety} $5 /$ - to $10 / 6$ each

Tàmarix (Tamarisk)

gallica

germanica

(Myricaria)

) $1 /$ - each ; 9/- per doz.

hispida æestivalis $\quad-\quad 16$ odessana

parviflora $-1 /-$

tetrandra (taurica) 1/- each. and others.

Tilia (Lime)

$\begin{array}{lrr}\text { alba (argentea) } & \mathbf{2} / \mathbf{6} \text { to } \mathbf{5} /- \\ \text { americana } & \mathbf{2} / \mathbf{6} & \mathbf{5} /- \\ \text { dasystyla (euchlora) } & \mathbf{3} / \mathbf{6} & \mathbf{5} /- \\ \text { europaa (vulgaris) } & \mathbf{1} /- & \mathbf{7} / \mathbf{6} \\ \text { Oliveri } & - & \mathbf{2} / \mathbf{6}\end{array}$

The heart-shaped leaves of this distinct Lime are glabrous on the upper surface and covered beneath with a white tomentum.

petiolaris $\quad 3 / 6$ to $10 / 6$

platyphyllos $\quad 1 / 6 \quad 5 /-$

- laciniata (aspleniifolia) 1/6 2/6

- rubra $1 / 6 \quad 3 / 6$

UImus (Elm)

campestris

(English Elm) 1/6 5/-

- Dampieri aurea $2 / 6 \quad 3 / 6$

- Louis Van Houtte 2/6 5/-

- Rosseelsii

(antarctica aurea) $\quad 3 / 6 \quad 5 /-$

- viminalis

variegata $3 / 6 \quad 5 /-$

glabra vegeta

$$
\text { (Huntingdon Elm) } \quad 1 / 6 \quad 2 / 6
$$

- cornubiensis

$$
\text { (Cornish Elm) 1/6 5/- }
$$

montana

(Scotch or Wreh Elm) 1/- 3/6

- fastigiata $1 / 6 \quad 2 / 6$

- pendula $3 / 6 \quad 21 /-$ 


\section{GENERAL COLLECTION (continued)}

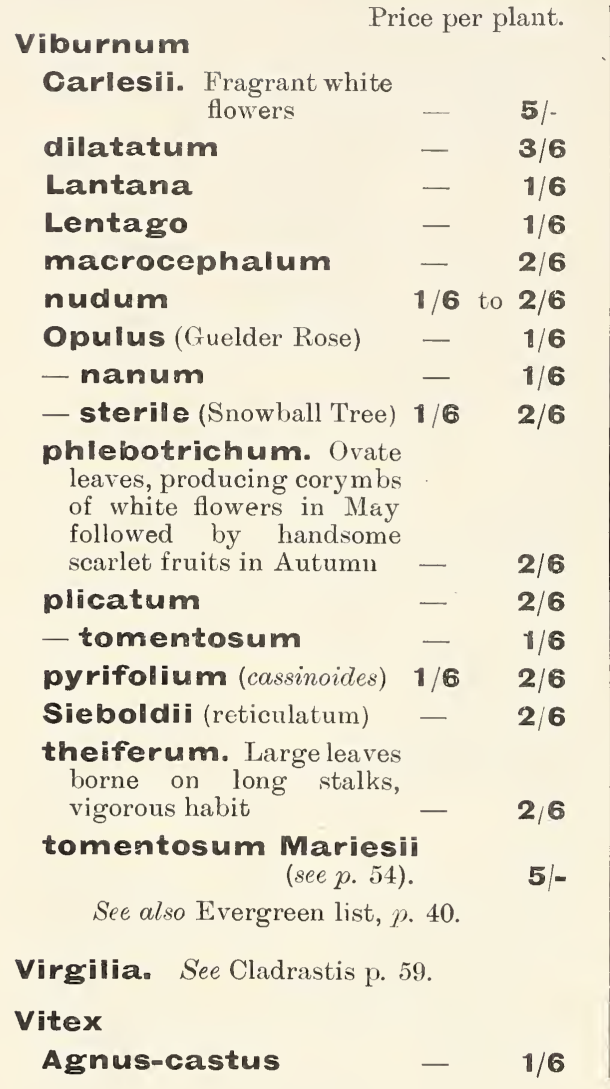

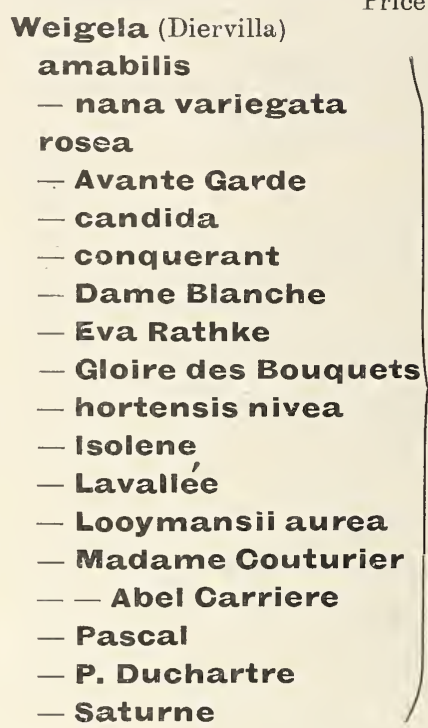

Zelkova (Planera) acuminata (Keakii) $\quad 1 / 6$ to 2/6 


\section{NEW AND CHOICE CLIMBERS.}

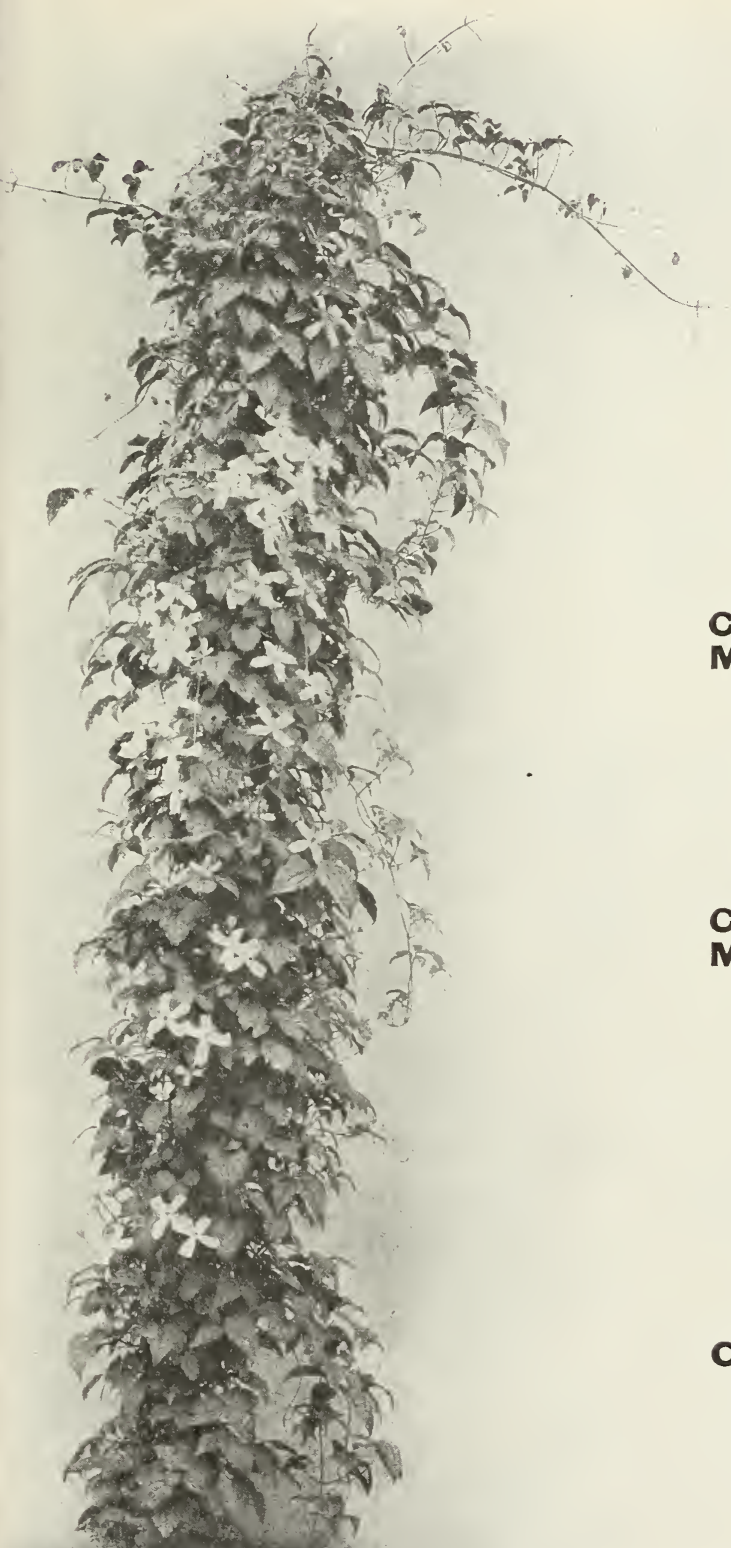

\section{ACTINIDIA CHINENSIS.}

A woody climber with heartshaped leaves and bright yellow flowers $1 \frac{1}{2}$ inches in diameter, borne very freely on ripened growths. Most useful as a pillar or pergola plant. A.M. R.H.S.

2/6 and 3/6 each.

\section{CLEMATIS ARMANDII.}

An evergreen species with dark green leaves and pure white flowers, 2 inches in diameter, borne in dense axillary clusters. Flowering early in April. Suitable for pergolas (see illustration p. 76) $\mathbf{3} 6 \mathbf{6}$ each. Fig. in Bot. Mag. T. 7897.

\section{CLEMATIS MONTANA RUBENS.}

A very effective decorative climber. In foliage and habit resembling the wel! known type with this remarkable difference, the flowers are of a soft rosy-red colour, and very freely produced (see illustration p. 77). F.C.C. R.H.S. $1 / 6$ \& $2 / 6$ each.

\section{CLEMATIS \\ MONTANA WILSONII.}

A very fine introduction from China, resembling $\mathrm{C}$. montana in growth and floriferous habit, but the individual flowers are much larger, being 2 to 3 inches in diameter, pure white in colour. It has the great merit of blooming in August, a time when flowering climbers are scarce. We highly recommend it as an excellent subject for pillars and pergolas (see illustration).

2/6 and $3 / 6$ each.

\section{CLEMATIS NUTANS.}

A perfectly hardy sub-shrubby climber. The cream-white, bellshaped pendulous flowers are very freely produced in clusters during July and August. Suitable for pillars or pergolas. $\quad$ 2/6 each.

\section{JASMINUM PRIMULINUM}

A magnificent species, with bright yellow flowers, much larger than those of J. nudiflorum, and produced in great profusion during spring. Although not quite hardy it is a decided acquisition to our gardens, being especially suitable as a wall plant, in any very sheltered situation. F.C.C. R.H.S.

1/6 and 2/6 each. 


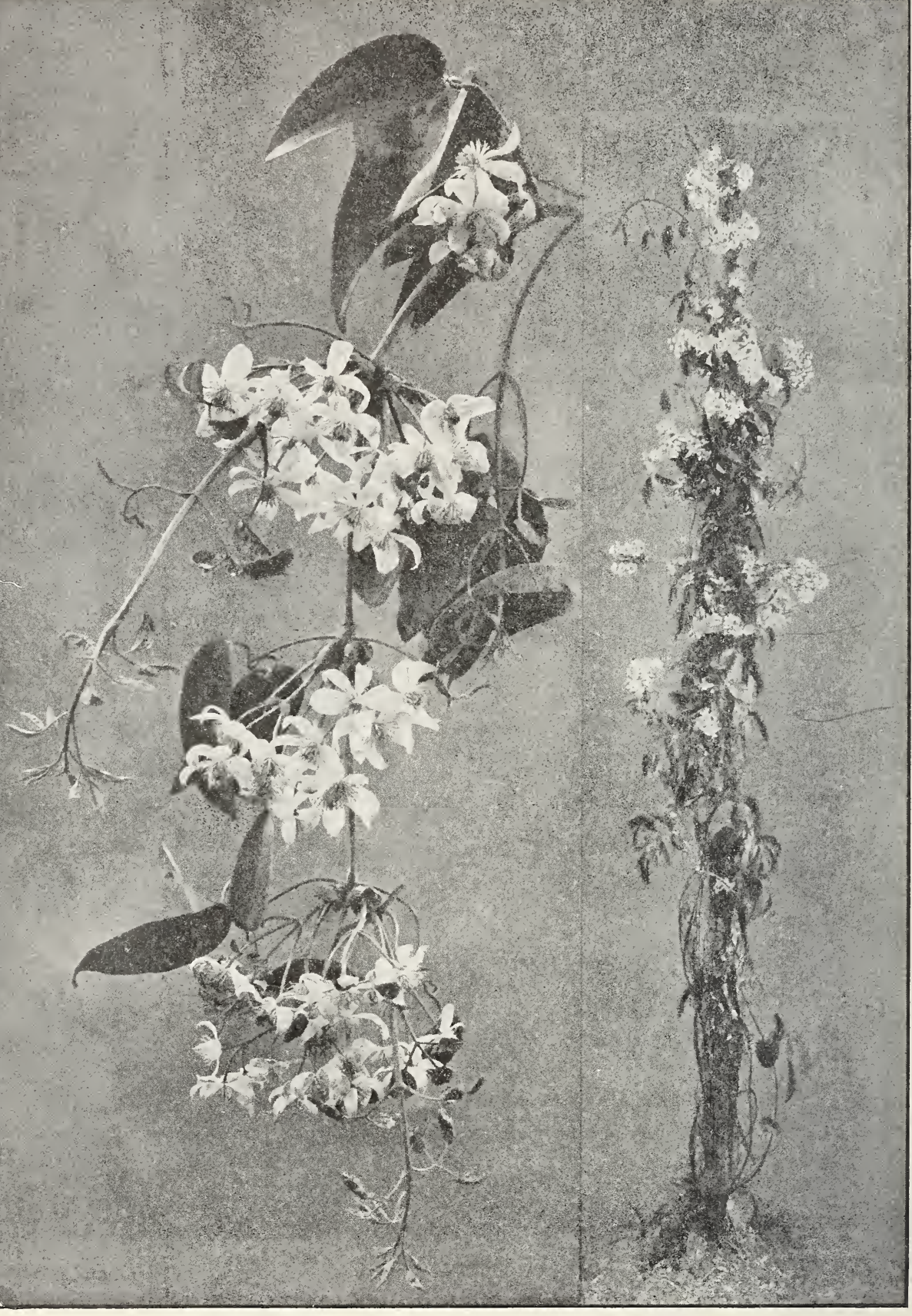

Olematis Armandii (see page 75). 


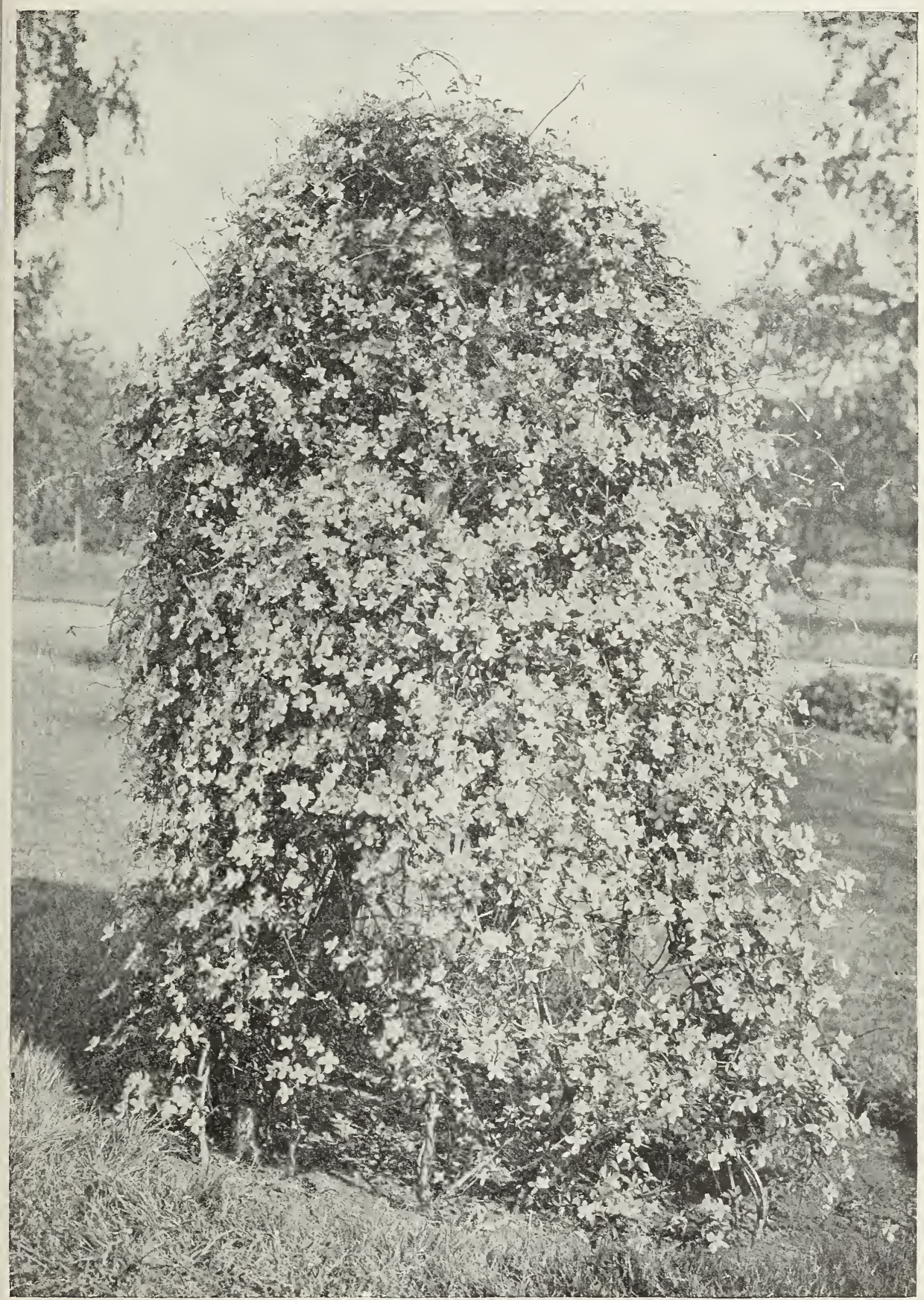

Clematis montana rubens (see page 75 ). 


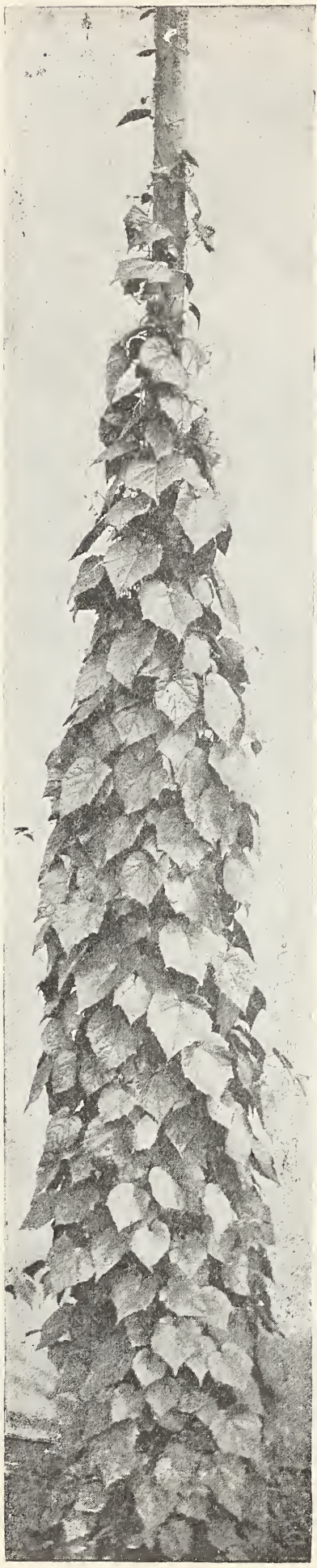

Vitis armata.

\section{VITIS ARMATA.}

A valuable acquisition for ornamental planting. The leaves are dark green in summer, changing to various bright red tints in the autumn (see illustration). A.M. R.H.S.

2/6 each.

\section{VITIS ARMATA VEITCHII.}

The finest decorative vine ever distributed, being more vigorous and in every way superior to the now well-known type. It succeeds well in any situation. The autumn tint of its large leaves is a rich crimsonlake, with a magenta shading, whilst during summer they present a shining bronze-green appearance (see illustration p. 80). A.M. R.H.S.

$3 / 6$ and 5/- each.

\section{VITIS FLEXUOSA WILSONII.}

A distinct form of the species of much ornamental value. The very neat foliage is remarkable for its deep bronzy hue and shining metallic lustre, while the under surface of the young leaves is of a bright purple colour. A.M. R.H.S.

$2 / 5$ and $3 / 6$ each.

\section{VITIS HENRYANA.}

The ground colour of the leaves is a dark velvety green, whilst the midrib and principal veins are silvery white. This variegation is more pronounced in autumn when the ground colour changes to red, the midrib and veins remaining white. A.M. R.H.S.

$1 / 6,2 / 6$ and $5 /$ - each.

\section{VITIS LEEOIDES.}

A distinct and most attractive vine, having pinnate leaves resembling those of the genus Leea, composed of five ovate-oblong leaflets of a rich glossy green on the upper surface and deep claret colour beneath, glabrous in all parts. A.M. R.H.S.

$3 / 6$ and $5 /-$ each.

\section{VITIS MEGALOPHYLLA.}

A remarkable species of Vitis, with bipinnate leaves 2 or 3 feet across, resembling individually those of Koelreuteria paniculata. They are dark green on upper surface, pale glaucous green beneath, and glabrous in all parts. A valuable addition to climbing plants, making growths of 8 to 10 feet in a season.

A.M. R.H.S.

$2 / 6$ and $3 / 6$ each.

\section{VITIS SINENSIS.}

A species with very variable bright green leaves, some simple broadly ovate, more or less trifid, whilst others are cut into 3 or 5 distinct leaflets. Suitable for Pergolas.

2/6 each. 


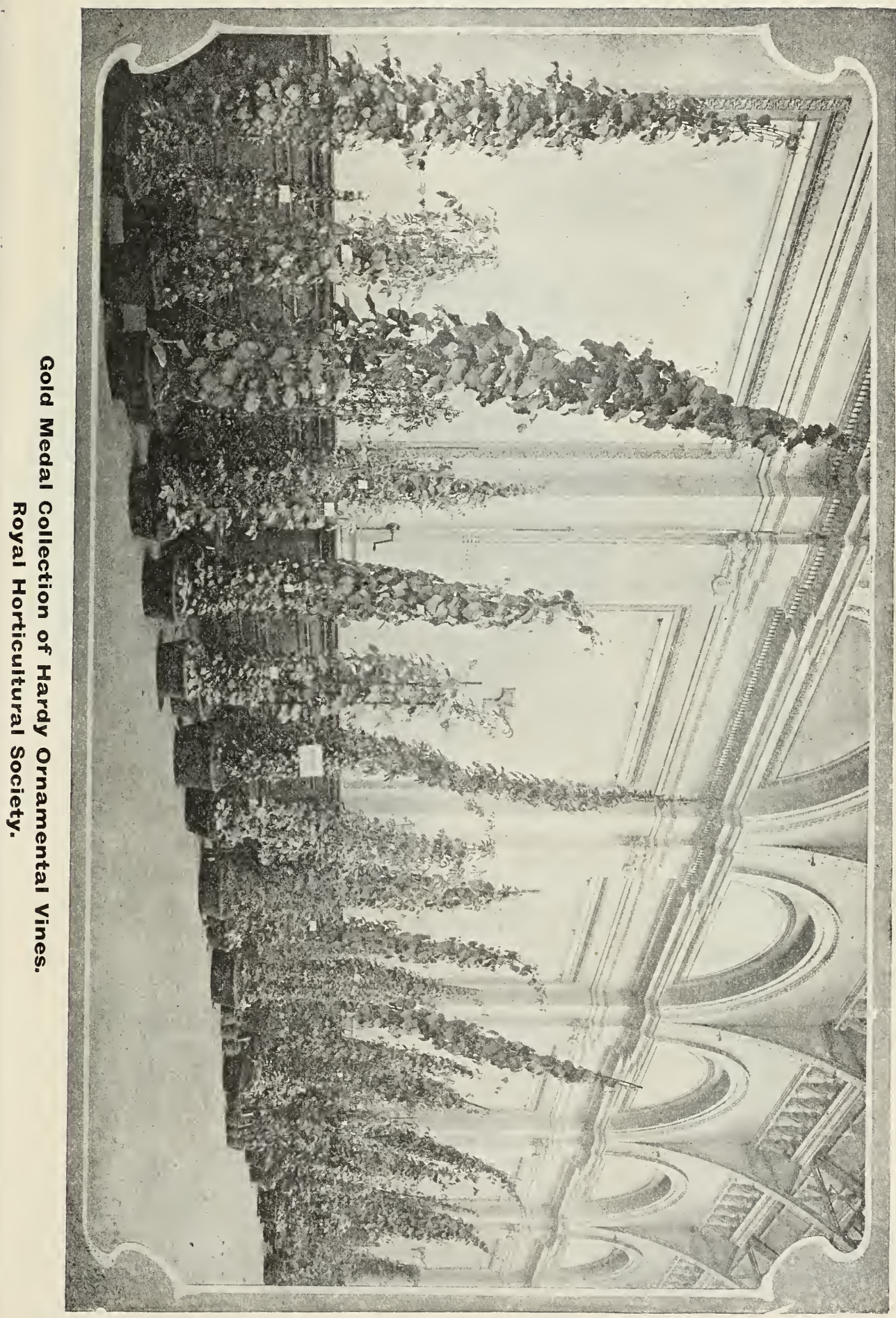




\section{VITIS REPENS.}

A fine hardy ornamental vine. It is self-clinging and a very strong grower. The young growths are bright brown in colour, the young leaves being of a reddish brown colour on the upper surface and deep claret colour beneath. The older leaves are $\mathbf{5}$ to 6 inches long, slightly trilobed, deep green and with a peculiar velvety appearance.

$2 / 6$ each.

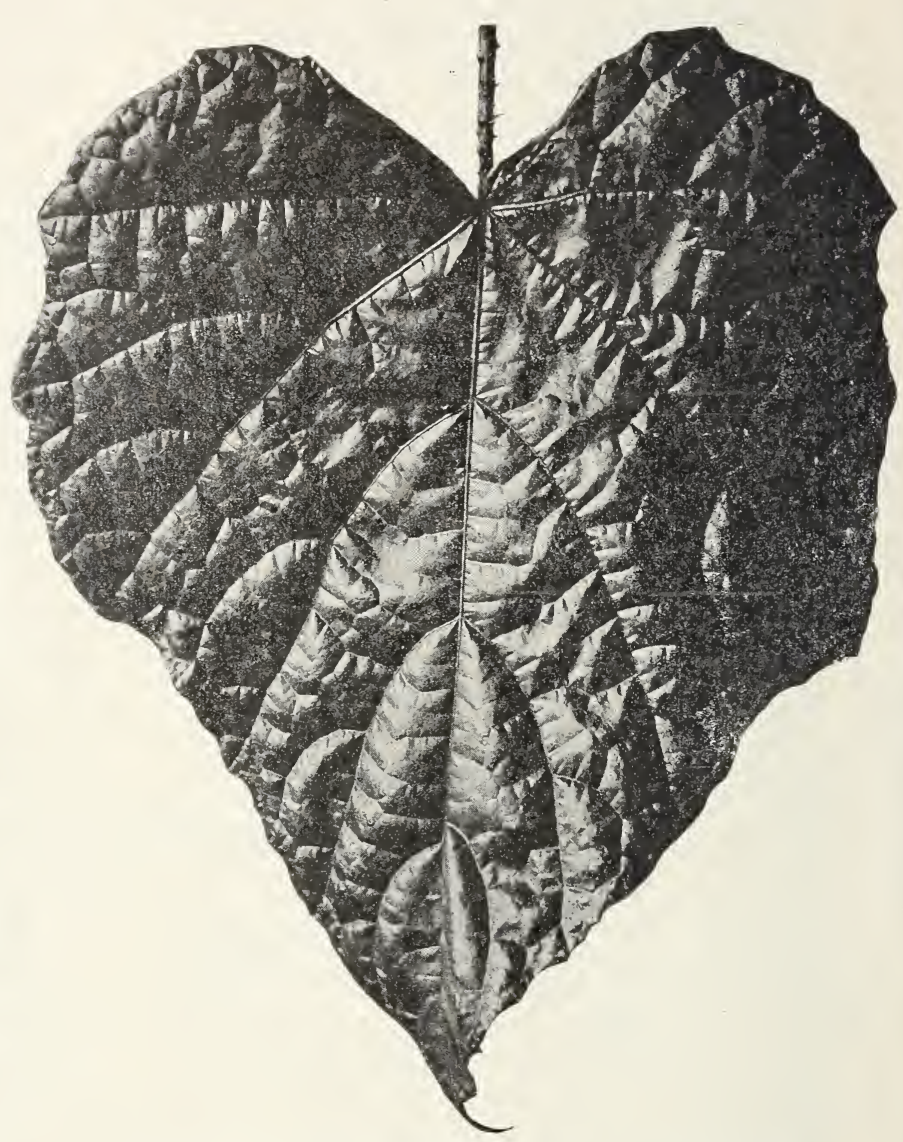

Vitis armata Veitchii (see page 78)

$3 / 6$ and $5 /-$ each.

\section{VITIS THOMSONII}

A charming species with purplish foliage and stems. The leaves are composed of five leaflets, greenish. purple above, bright claret colour beneath, changing in autumn to purplish-red. The growths are slender and the plant is admirably adapted for situations where stronger kinds would be too vigorous. A.M.R.H.S.

$1 / 6,2 / 6$ and 5/- each. 


\section{GENERAL COLLECTION.}

Actinidia arguta

Price per plant.

chinensis

$1 / 6 \quad 2 / 6$

Kolomikta

2636

1626

Akebia

quinata

16

Ampelópsis

Engelmanni

$1 / 6$

26

hederacea (quinquefolia) (Virginian Creeper) 1/=

- muralis

$1 / 6$

$2 / 6$

Lowii

$1 / 6$

Veitchii (tricuspidata) 1/- 2/6

- purpurea

sempervirens

(Vitis striata)

See also Vitis page 85.

\section{Aristolòchia}

\section{moupinensis}

Of rapid growth reaching 12 to $15 \mathrm{ft}$. in a season, flower tube about $2 \frac{1}{2}$ inches in length, yellowish green with purple markings. Suitable for pillars and pergolas.

\section{Sipho}

\section{Berberidòpsis}

*orallina

Bignónia (Tecoma)

\section{grandiflora}

radicans

- sanguinea

- Thunbergii

\section{Caprifolium}

See Lonicera page 84.

\section{Celàstrus}

\section{hypoglaucus}

A deciduous species with large leaves, deep pea-green above, glaucous beneath. Young wood covered with waxy bloum

\section{scandens}

\section{clèmatis}

1. Natural species and their varieties.

athusifolia

Very graceful, leaves small and divided up into narrow linear lobes.

Flowers not very large, whitish, bell shaped, very abundantly produced in September and October.

26
Clematis (continued) Price per plant. alpina (Atragene austriaca),

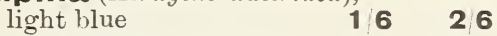

Armandii (see page 75) _ $\quad 36$

calycina, creamy white - $1 / 6$

coccinea, scarlet $\quad-\quad 16$

carulea odorata,

reddish violet, sweet

scented - 16

cirrhosa, white, evergreen - 16

crispa, pale lilac — $\quad 16$

Flammula, (Tirgin's

Bower), white - 16

- rubro-marginata, creamy white, red margin - $1 / 6$ grata

Bluish - white flowers gracefully disposed, and freely produced during the autumn. A.M.R.H.S.

graveolens, yellow

montana, white

$\begin{array}{lll}\text { - rubens (sce page } 75) & \mathbf{1} / 6 & 2 / 6\end{array}$

- Wilsonii (see page 75) $2 / 6 \quad 3 / 6$

nutans (see page 75) - 26

orientalis, white, sweet scented - $1 / 6$

- tangutica $\quad$ - $2 / 6$

Rich golden yellow flowers, produced on peduncles 6 in. long, gracefully arched at the tips.

paniculata (Flammula robusta)

Autumn - flowering, innumerable pure white stellate fragrant flowers.

Vitalba ('Traveller's Joy), white $1 / 6 \quad 2 / 6$

$\begin{array}{lll}\text { Viticella, bluish purple } & \mathbf{1} / 6 & \mathbf{2} / 6\end{array}$

- alba, greyish white - 16

- Kermesina, wine red - $1 / 6$

- grandiflora punicea, red - $1 / 6$

2. Coccinea hybrids.

Countess of Onslow, bright violet purple - $\quad 16$

Duchess of Albany, bright pink, deeper centre - $\quad 1 / 6$

— York, pale blush pink — $1 / 6$

Grace Darling, bright rosy carmine - $1 / 6$

Sir Trevor Lawrence, bright crimson - $2 / 6$ 


\section{GENERAL COLLECTION (continued).}

Clèmatis (continued)

3 Garden hybrid.s.

Admiration, fine deep salmon alba magna, pure white Beauty of Worcester, bluish violet

Belle Nantaise, fine lavender

Blue Gem, deep lavender blue

Belle of Woking, silver grey, double

Colette Deville, dark red

Comtesse deBouchard soft carmine rose

Countess of Lovelace, bluish lilac, double

Duchess of Edinburgh, double white, sweet scented

- - Teck, pure white, mauve bar

Duke of Edinburgh, rich violet purple

Edith Jackman, delicate white flushed with mauve

Edouard Desfosse, a deep shade of mauve

Elsa Späth, dark purple

Enchantress,double white flushed with rose

Excelsior, deep mauve

Fairy Queen, pale flesh, pink bar

Gem, deep lavender blue

Guiding Star, purple, shaded crimson

Henryi, creamy white

Jackmanii, violet purple

- nivalis (alba), pure white

- rubra, bright red

- superba, dark violet purple

Jeanne d' Arc, greyish white

John Gould Veitch, lavender blue, rosette form

King Edward Vll., violet, crimson bar

Lady Boville, greyish blue, cupped

- Northcliffe, deep lavender

- Caroline Neville, French white, mauve bar

La France, deep violet purple, dark anthers each $3 / 6$
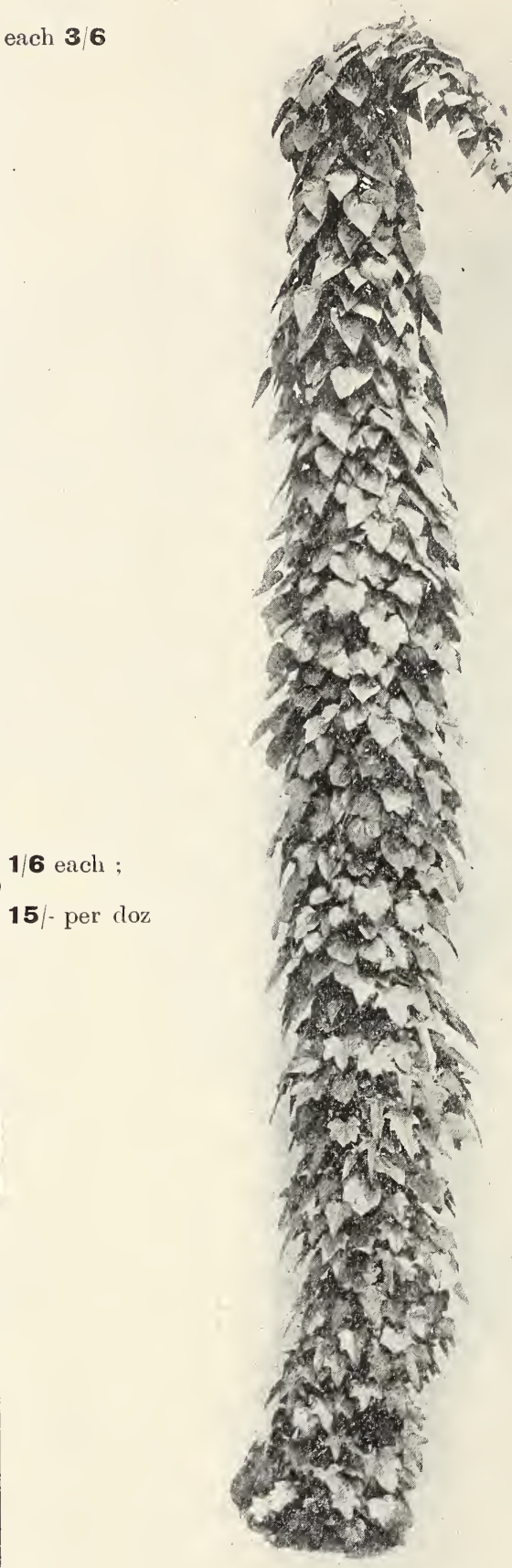

Cocculus heterophyllus (see page 83 ). 


\section{GENERAL COLLECTION (continued).}

Clèmatis, (continued).

La Lorraine, clear rose tinted with blush

Lanuguinosa, pale lavender

Lawsoniana, rosy purple, darker veins

Lord Beaconsfield, dark plum

- Gifford, deep pink

- Neville, bright blue

Lucy Lemoine, white rosette formed

Madame Baron Veillard, light lilac rose

- Edouard André, bright red

- Grange, crimson violet

- Jules Correvon, very rich red

- Le Coultre, fine white

- Van Houtte, white suffused with mauve

magnifica, reddish purple

Marcel Moser, pale violet distinct red bar

Marie Lefebvre,delicate mauve

Miss Crawshay, pink, distinct

Mrs. Cholmondeley, lavender tipped with purple

- Hope, satiny mauve

Mrs. G. Jackman, satiny white, creamy bar

Nelly Moser, light maure with red bar

- Koster, pure white

Oriflamme, violet red

Othello, dark velvety purple Otto Fróebel, greyish white

Palmyre, rosy carmine

President, purple, suffused with claret

Prince of Wales, deep purple

Princess of Wales, deep bluish mauve

purpurea, elegans, deep violet purple
Clèmatis (continued)

Queen Alexandra, pale lavender

each 36

Rubella, rich claret purple

Sir Garnet Wolseley, blue, plum-red bar

Star of India, reddish plum, red bar

Symeana, pale mauve

Tunbridgensis, deep bluish mauve

velutina, purpurea, dark purple

Venus Victrix, pale lavender

1/6 each ; $15 /$ - per doz.

Ville de Lyon, rich carmine crimson

- Paris, pale flesh, pink bar

William Kennett, deep lavender

\section{Cocculus}

Price per plant.

heterophyllus, a deciduous climber of rapid growth, particularly suitable for pillars and pergolas. See illustration page 82
16 each ; 15 - per doz.
Thunbergii, an evergreen climber of free growth, leaves deep green, ovate lanceolate. Suitable for pergolas

Ercilla (Bridgesia)

spicata

Hèdera (Iry)

Helix (Common English Ivy), 12/ - \& 18/-per doz.

- acuta

- algeriensis

- angularis

- - aurea

- argentea rubra

- atropurpurea

- aurea

- densa

- azorica

- Cxnwoodiana

- canariensis (Irish

$1 / 6$ each

15 - \& 18

\begin{tabular}{l|c} 
Iatifolia & \multicolumn{1}{c}{ Ivy) } \\
maculata & $\begin{array}{c}\text { Extra strong } \\
\text { plants, } \\
\text { 2/6 each; } \\
\text { - chrysophylla } \\
\text { - conglomerata } \\
\text { - contracta } \\
\text { - dentata }\end{array}$
\end{tabular} 


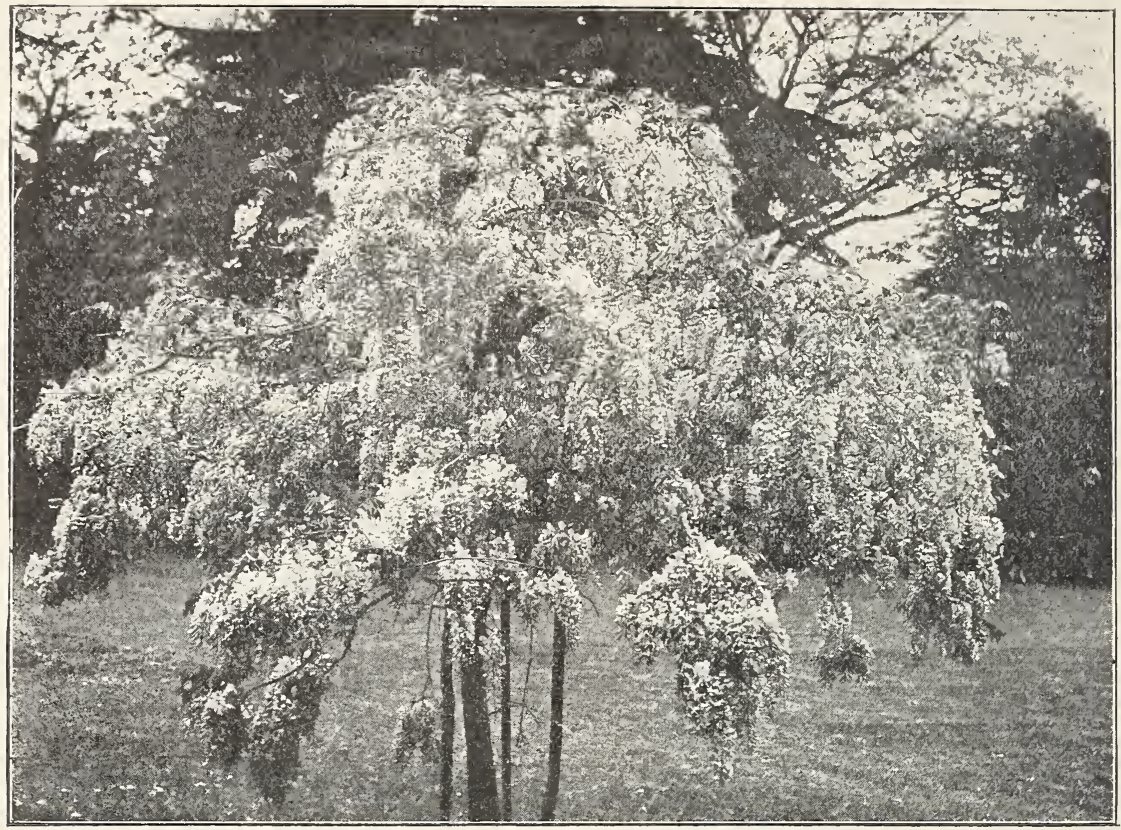

Wistaria sinensis (see page 85).

Hèdera (continued) Helix dentata

- digitata

variegata $2 / 6$ to 5 -

- - aurea

- Emerald Green

- gracilis

- himalaica

- Iatifolia argentea

- maculata

- maderensis

- palmata variegata

- - aurea

- poetica

- Roegneriana

- rhomboidea ovata

- sagittafolia

- spectabilis aurea

- variegata argentea

- elegantissima

Price per plant.

Irish Ivy, in pots, $100 /-$ per 100 , and upwards; out of pots, $\mathbf{3 0} /-$ to $\mathbf{5 0} /-$ per 100 .

$1 / 6$ each ;
$15 /-\& 18 /-$ doz.

Extra strong

plants,

2/6 each ;

30/-per doz.

\author{
Jasminum \\ fioridum (subulatum) \\ fruticans \\ nudiflorum \\ — folis aureis \\ ochroleucum \\ officinale (sweet white) \\ - foliis aureis \\ *primulinum (see page 75). \\ * revolutum \\ *Wallichianum $\int 12 /-\& 18 /-\mathrm{doz}$. \\ 1/6 each ; \\ $12|-\& 18|$ \\ doz. \\ Price per plant.
}

Lardizâbala

* biternata

Lonicèra (Caprifolium)

brachypoda

- aureo-reticulata

flava (Yellow Trumpet)

fiexuosa

gigantea

Hildebrandtii
$2 / 6 \quad 3 / 6$

1/6 each ;

$12 /-\& 18 /=$

doz.

- 2/6

$3 / 6$ to $5 /=$

* Require a South West aspect. 
GENERAL COLLECTION (continued)

Lonicera (continued).

japonica (Halliana)

Periclymenum (Common Honeysuckle)

- Early Cream

$1 / 6$ each ;

$12 /-\& 18 /=$ doz.

\section{- Dutch}

- Late Dutch

Extra

strong, 2/6

sempervirens (Scarlet) \& $\mathbf{3} / \mathbf{6}$ each.

Menispérmum

Price per plant. canadense

$1 /$ - to $1 / 6$

Muehlenbeckia complexa

$-1 / 6$

varians

$1 / 6$

Passifióra (Passion Flower)

* corulea

$1 / 6$

$2 / 6$

*-Constance Elliott 1/6

Periploca

graca

$1 / 6$

2/6

Baldschuanicum growth; the stems are twining and cling for support to any object within reach. Every branchlet terminates in a panicle of white flowers, which are produced in the greatest profusion from June to September.

\section{multiflorum}

An ideal plant for covering arbors, trellises, dead trees, etc. The long rampant growths, often attaining the length of from 20 to 30 feet in one season, produce a highly ornamental effect when allowed to ramble at will.

vaccinifolium

Smilax

aspera

- variegata

caduca (rotundifolia)

sagittæfolia

- variegata

\section{Stauntonia}

*hexaphylla

$1 / 6$

* Iatifolia(Holballia latifolia) 1/6

$2 / 6$

$2 / 6$

Vitis

\section{aconitifolia}

armata (see $p .78$ )

- Veitchii (see $p$. is)

26

$2 / 6$
Vitis (continued)

restivalis

citrulloides

Price per plant.

\section{Coignetia}

- 1/6

$-1 / 6$

targe thick leaves, often

9 to $10 \mathrm{in}$. long and as much broad, rich deep green above, covered with a soft woolly tomentum of a pale buff yellow beneath. In autumn the leaves assume brilliant shades of yellow, orange red, purple and brown crimson.

flexuosa

$2 / 6$

- major

$3 / 6$

- Wilsonii (see p. 78).

Henryana (see p. 78).

heterophylla

- dissecta

- humulifolia

- striata

- variegata

Labrusca

5 -

$-\quad 1 / 6$

$-\quad 1 / 6$

$-\quad 1 / 6$

$-\quad 1 / 6$

leeoides (see $p .78) . \quad 3 / 6 \quad 5$.

megalophylla(see $p$. 78) $2 / 6 \quad 3 / 6$

odoratissima $\quad$ - $1 / 6$

orientalis (Cissus) _ $\quad$ 1/6

repens (see p. 80). $\quad \mathbf{2 / 6}$

sinensis (see p. 78) $\quad$ 2/6

Thomsonii (see p. 80). $\mathbf{1 / 6} \quad \mathbf{5}$ -

Thunbergii - 5/-

The foliage in autumn assumes the most brilliant hues of scarlet and crimson. A strong and free grower when well established.

$\begin{array}{lll}\text { vinifera (grape vine) } & 1 / 6 & 2 / 6\end{array}$

- Aramon teinturier Bouschet - $3 / 6$

- apifolia

$1 / 6$

- laciniata

- purpurea vulpina

Wistaria (Glycine)

sinensis

5 (see illustration p. 84)

- (Standards)

$7 / 6$

- alba

5 -

$5 /-$

- aurea reticulata - 3/6

- fiore pleno $2 / 6 \quad 5 /=$

- multijuga (true) - $\mathbf{5}$ /= (see illustration p. 86)

- - (Stundards)

$7 / 6$

- alba

- - (Standards)

5/- 


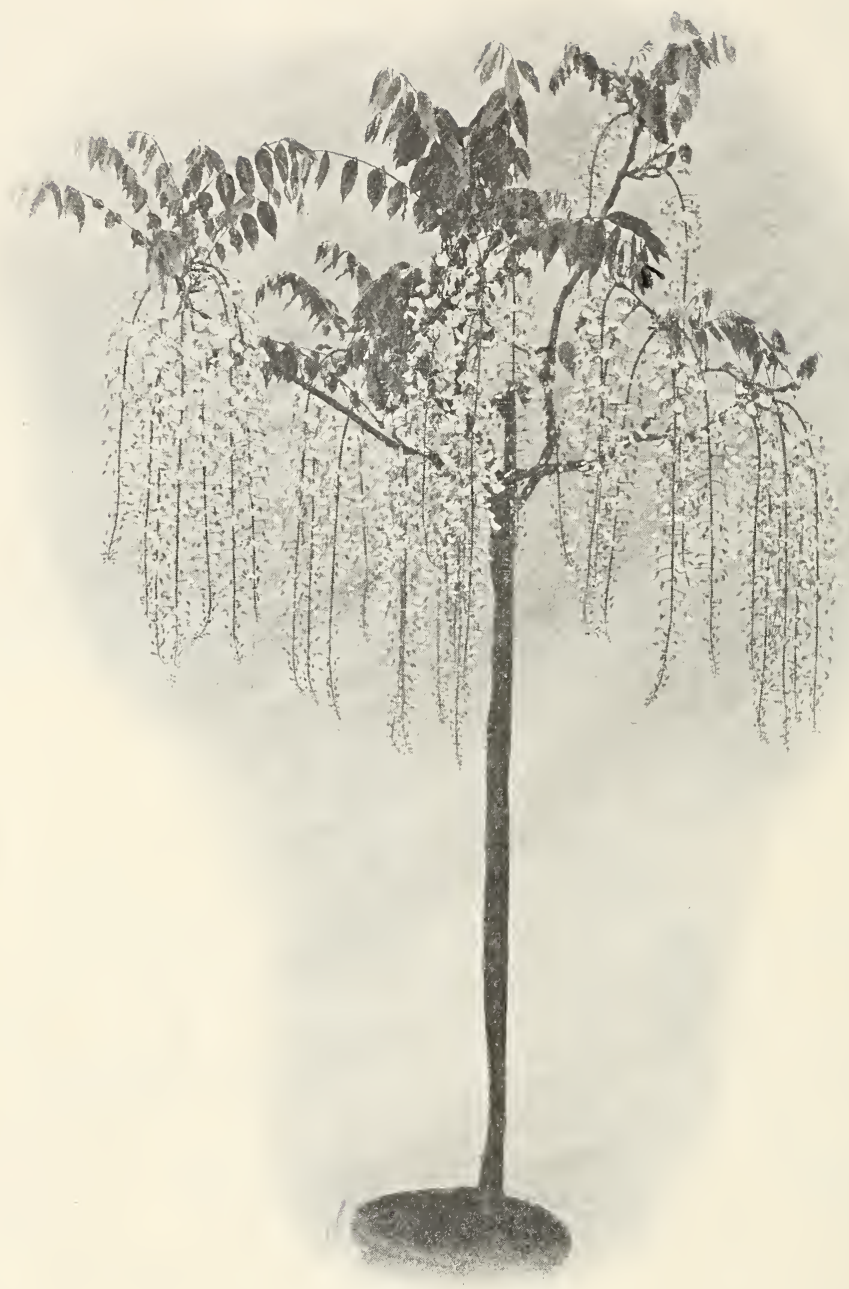

Standard Wistaria multijuga, $7 / 6$ each. Remarkable for its very long racemes (see page 85). 


\section{PLANTS FOR COVERING BUILDINGS, \&C.}

Supplied in pots. Those marked $\left(^{*}\right)$ require a south or west aspect.

Ampelópsis (see paye 81).

Price per plant

Azara

Gilliesii

$-26$

* Ianceolata

microphylla

*serrata

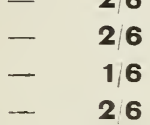

Berberis

stenophylla

16

26

Berchèmia

* racemosa variegata 26

36

Bùddleia

*Colvilei

globosa

intermedia

Lindleyana

variabilis

- 16

- 16

$-\quad 16$

- 16

- magnifica (see page 45) 2/6 3/6

- Veitchiana (see page 45) $1 / 6 \quad 2 / 6$

Calycànthus

foridus

macrophyllus

1626

pracox

Camellia

Sasanqua, double

- double white

pink

- single red

\section{Carpentaria}

californica

Caryópteris

* Mastacanthus

* albus

Ceanothus

*azureus albidus

* Arnoldii

*-Brilliant

* Ceres

* Croix du sud

* Gloire de Plantierés

* - de Versailles

* Indigo

* -Marie Simon

* Pinquet Guindon

*dentatus (floribundus)

* divaricatus

*papillosus

*thyrsiflorus griseus

*Veitchianus

- 16

$-26$

$-\quad 26$

- 26

- 26

-

26

1626

- 26

- 16

- 26

- 16

- 16

- 26

$-\quad 26$

$2 / 6$

2/6
365

Chimonànthus

Price per plant.

* fragrans

16 to $2 / 6$

Choisya

ternata

$16 \quad 3 / 6$

Coronilla

Emerus

Cotoneàster

angustifolia

applanata (see paye 45)

buxifolia

Hookeri

horizontalis

microphylla

rupestris

refiexa (see paye 45)

Simonsii

Cratagus

Pyracantha (north or

\begin{tabular}{|c|c|}
\hline east aspect) & 16 \\
\hline - crenulata & 16 \\
\hline - Lælandii & 16 \\
\hline
\end{tabular}

Cydònia (Pyrus)

japonica

- atropurpurea

- cardinalis

- coccinea

- flore albo

- Moerloesii

- nivalis

- rosea

- Simonsii

Maulei

- superba

- 16

- 16

- 26

- $1 / 6$

- $1 / 6$

26

- 16

- 16

$162 / 6$

- $1 / 6$

- 26

Desmodium

nutans

pendulifiorum

26

Elaàgnus

glabra (reflexus) _ $\quad 1 / 6$

— folis variegatis _ $\quad 1 / 6$

$\begin{array}{lll}\text { macrophyllus } & 16 \quad 26\end{array}$

$\begin{array}{lll}\text { pungens } & 16 & 2 / 6\end{array}$

$\begin{array}{lll}\text { - variegatus } & 16 & 2 / 6\end{array}$

- aureo-maculatus $16 \quad 26$

$\begin{array}{lll}\text { - pictus } & 16 & 2 / 6\end{array}$

Embothrium

*coccineum $\quad 5$ - 106

Eriobotrya (Loquat Tree)

*japonica

26 to 5 


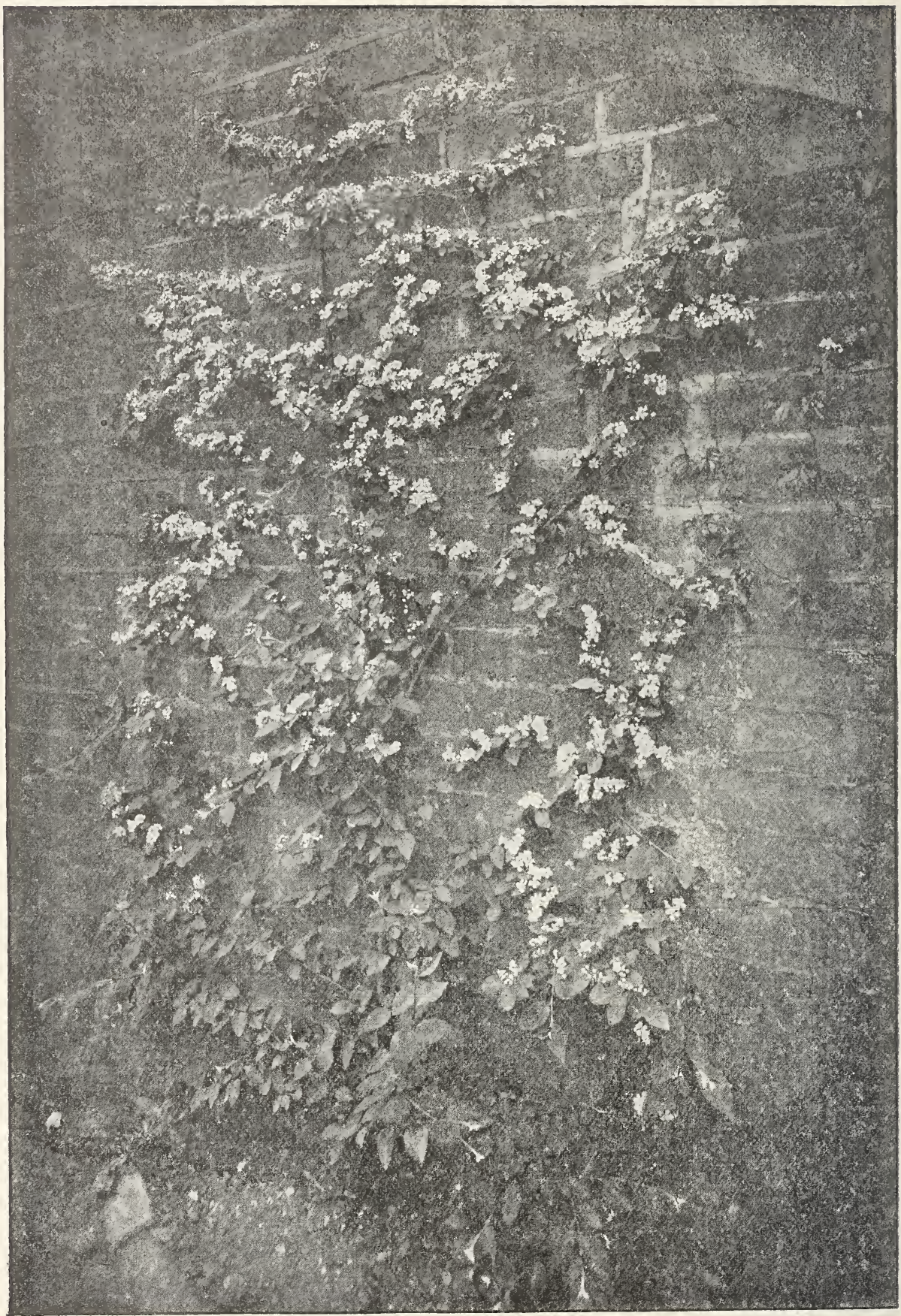

Cotoneaster reflexa (see page 45). 
* require a South or West aspect.

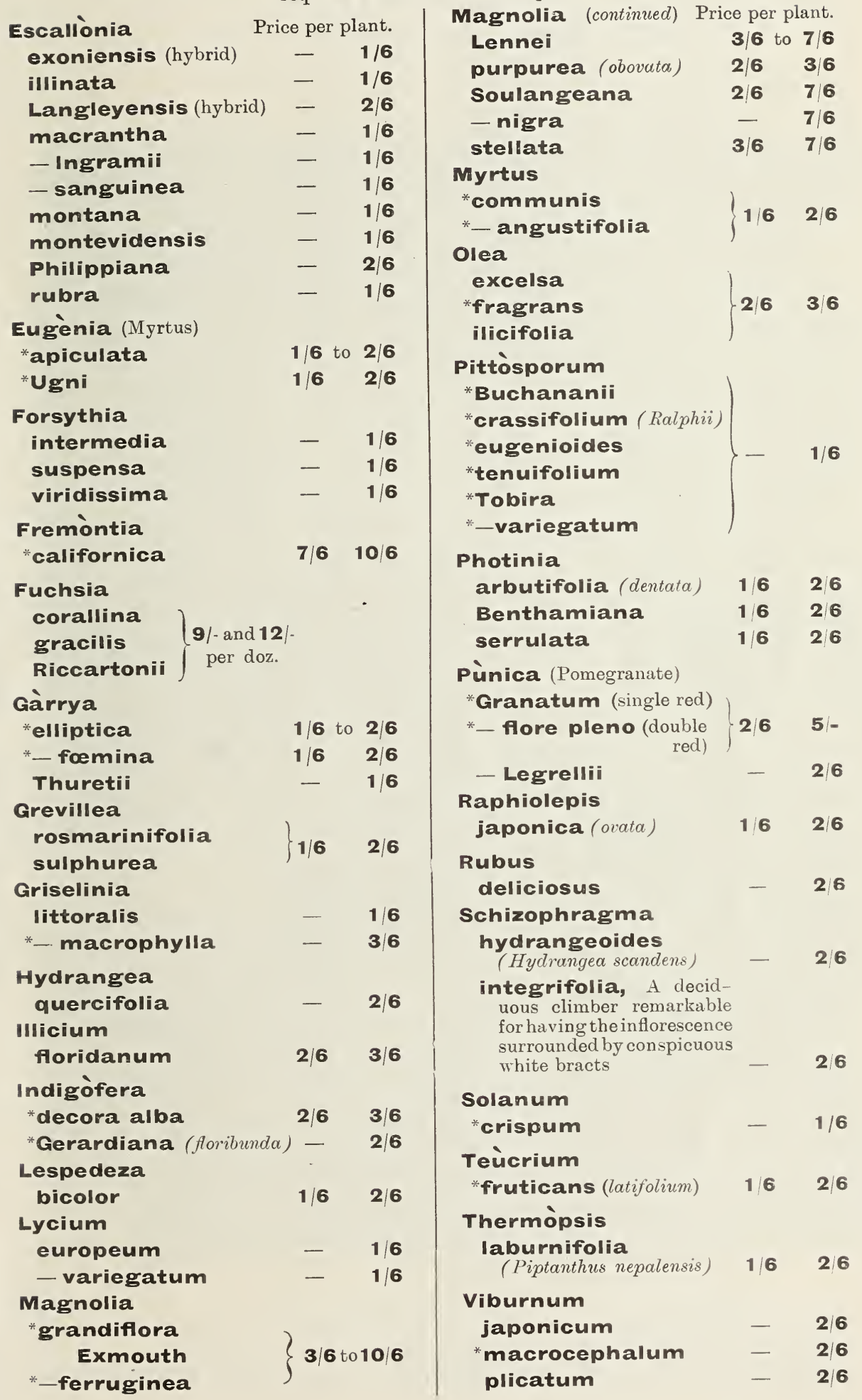


Acorus Calamus (Sweet Flag), long green foliage, yellow aromatic flowers

9d.

, graminea variegata, green foliage with white margin

, japonica argentea striata, leaves striped with white

Alisma natans, an effective floating plant with pretty white flowers

"Plantago, heads of white flowers very graceful, height 4 feet

Aponogeton distachyon, this is not only hardy, but wonderfully free blooming for small Aquariums, as well as streams or pools. Its fragrance is most refreshing, reminding one of Hawthorn

Brasenia peltata (Schreberi), (Water Shield), small coppery peltate foliage and brownish - purple flowers very pretty

Butomus umbellatus, (The Flowering Rush), bright pink, numerous flowers, in large heads, with rush-like green leaves, height 5 feet

Calla palustris, (Water Arum), white flowers, bright green leaves, height 9 ins.

Caltha palustris, (Marsh Marigold), suitable for the margins of streams, producing abundance of golden-yellow flowers

Caltha palustris, fi. pl., a double flowered form, free-flowering

Carex pendula, a tall graceful plant with long drooping leaves

Cyperus Iongus, long graceful foliage, large heads of brown spikelets

Epilobium hirsutum, large rosy purple flowers

Glyceria aquatica variegata, a variegated Reed-like perennial

Hottonia palustris, (Water Violet), submerged fern-like plant with showy white flowers and bright green foliage

Hydrocharis Morus-ranae, (Frog Bit), a floating plant with pretty white flowers

Iris Pseud-acorus, (Water Flag), fine yellow flowers

foliis var., handsome variegated foliage

Juncus Zebrinus, beautifully marked foliage

"spiralis, curiously twisted leaved

Limnocharis Humboldtii, distinct in charanter from any of our native plants, rich golden-yellow flowers, beautifully fringed, one of the most exquisite in cultivation

Menyanthes trifoliata, (Bog Bean), a free growing plant, pink and white hairy fringed flowers, resembling the flowers of the Horse-Chestnut

Myosotis palustris semperflorens, (Water Forget-me-not)

Orontium aquaticum, (Golden Club), golden-yellow flowers, in early summer, height 18 inches

Peltandra virginica, large arrow-shaped foliage white spadix

Poa aquatica, dark-green Reed-like perennial, $4 \mathrm{ft}$.

Polygonum amphibium, rose-red spikes of flowers

Pontederia cordata, free flowering, about $2 \mathrm{ft}$. high, tall tufts of heart-shaped leaves with spikes of closely set blue flowers

Ranunculus Lingua, large showy yellow flowers about 2 to 3 feet above the water, narrow glaucous lance-shaped leaves

9d.

Rumex Hydrolapathum, (Giant Water Dock), gigantic leaves, very conspicuous Sagittaria sagittaefolia, (Common Arrow Head), flowers white with dark coloured anthers

Scirpus lacustris, bright green foliage, 3 to 6 feet high

" " zebrinus, very elegant, leaves alternately barred with white and green

Sparganuim ramosum, bright green leaves, Burr-like flowers borne on stems $2 \mathrm{ft}$. high

Stratiotes aloides, (Water Soldier), a pretty floating Aquatic with spiny Pandanus-like leaves and pure white flowers

Typha angustifolia, (Lesser Reed Mace or Cat's Tail), a tall slender species with brown leaves and chocolate coloured heads

"Iatifolia, (Great Reed Mace), well known native plant of fens and rivers, grows 6 to 8 feet high

" minima, a scarce dwarf species, 12 to 18 inches high . 


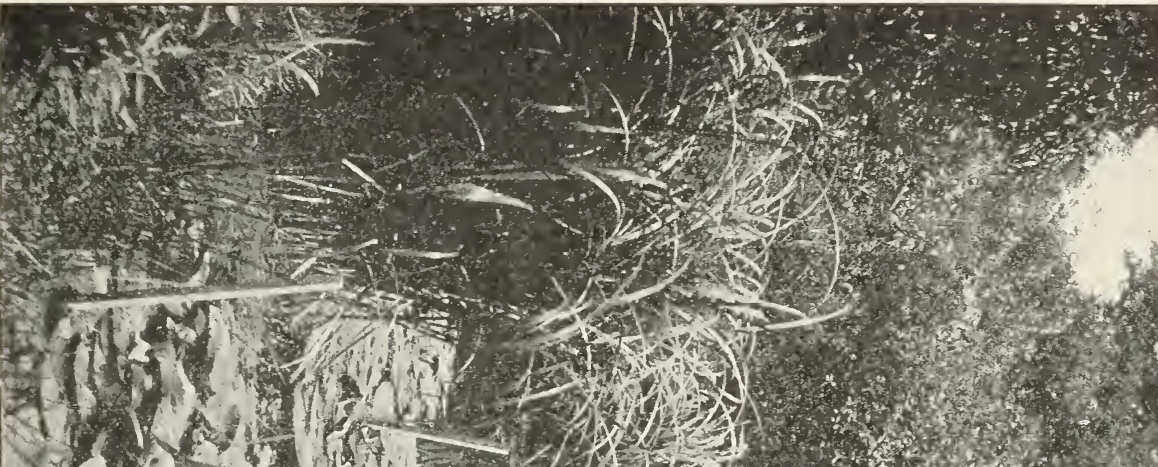

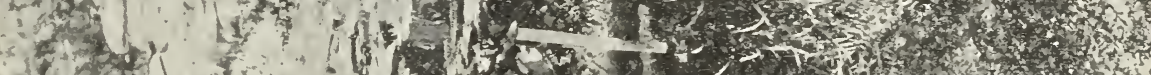
$1 \mathrm{a}$.

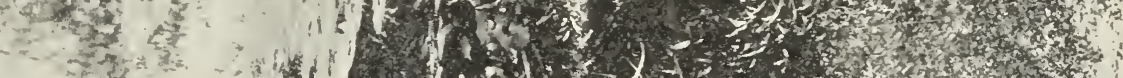

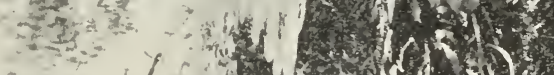
1)

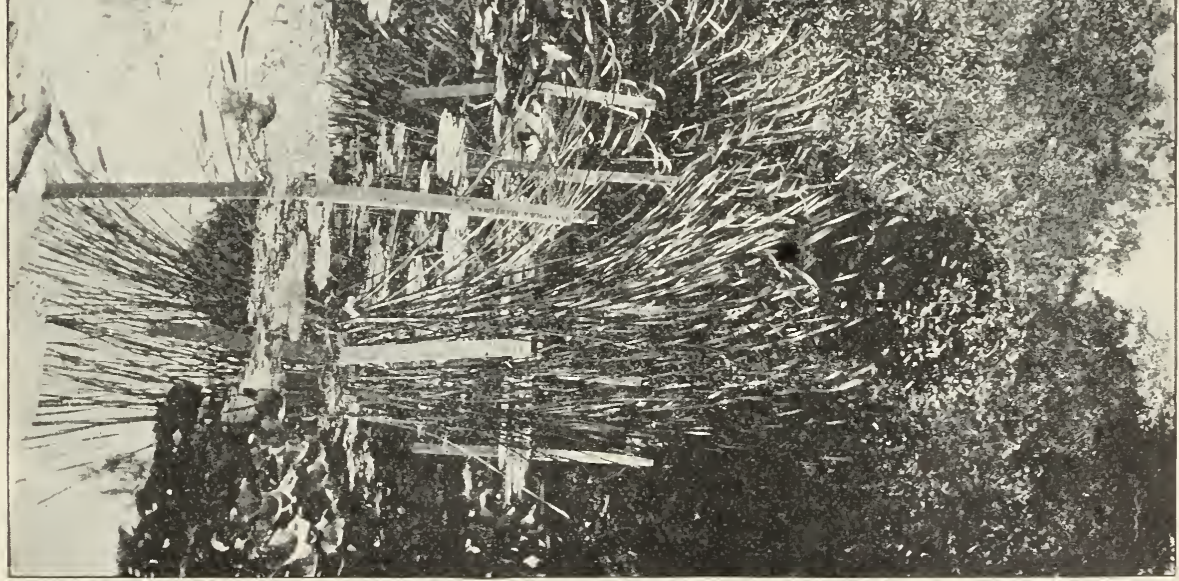




\section{EVERGREEN SHRUBS FOR BEDDING AND WINTER DECORATION.}

We beg to direct especial attention to the undermentioned Evergreen Shrulos, which are strongly recommended not only for the purpose of garden decoration in winter, but also for the formation of permanent beds, and for grouping. Their dwarf and compact habit, together with their capacity for being kept exceedingly dwarf by being pruned to almost any extent, and the many pleasing shades of colour that may be selected from amongst them, render them peculiarly suitable for carpet-like beds and other devices not less effective than those formed by summer-bedding plants. To the brilliant hues of these they offer an agreeable contrast, and at the same time introduce a new feature in summer-beding.

Andròmeda

floribunda

Price per dozen.

$\mathbf{1 8 / - ~ t o ~} 24 /$ -

Aucuba

japonica mascula $12 /$

- foemina

- vera nana

- Iongifolia

$\mathbf{9} /-\quad 12 /-$

Búxus

japonica aurea

sempervirens

suffruticosa

argenteo-mar-

ginata nova

Cotoneàster

horizontalis

microphylla

Cupréssus

Lawsoniana

- Allumii

- Iutea

- nana

- Shawii

nootkatensis

Erica

herbacea carnea

vagans alba

- carnea

vulgaris aurea and other sorts

Euónymus

japonicus

- aureo variegatus $9 / . \quad 12 /$.

- latifolius aureomarginatus

- radicans variegatus, per $100,30 /$. to 40 -

Hedera

arborea

- aurea

- elegantissima

$9 /-\quad 12 /-$

$6 /-\quad 9 /$.

6/- $9 /-$

$9 /-\quad 12 /-$

$9 /-\quad 12 /-$

6) 9

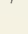

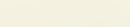

6/-

12/. $18 /$.
Ilex

crenata

Price per dozen.

Juniperus

tamariscifolia

Kalmia

latifolia

Ligustrum japonicum

Mah̀onia

aquifolium - 6/-

Osmanthus

ilicifolius

- variegatus
nanus
myrtifolius

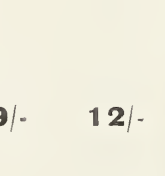

Pernettya

mucronata, and

varieties

Retinispora

plumosa

- argentea

- aurea

18 -

18 -

18/- to $30 /-$

$9 /-\quad 12 /$

$9 / . \quad 12 /$.

Rhododendron

arbutifolium

myrtifolium

ovatum

Wilsonii

18

Skimmia

Fortunei

japonica

9/- $12 /-$

Taxus

baccata elegantissima - 12/.

Thuia (Thuiopsis)

dolabrata

verónica

Traversii

6/- $\quad 9 /-$

Viburnum

Tinus (Laurustinus) $\quad 9 /-\quad 12 /$.

Vinca

elegantissima $\quad$ 4/- 
AUTUMN-TINTED FOLIAGE TREES AND SHRUBS.

Acer
circinatum
Ginnala (Tataricum
palmatum
- atropurpureum
- sanguineum
japonicum
colchicum rubrum
rubrum
saccharinum
plantanoides
Reitenbachii
- Schwedleri
Amelanchier
canadensis
Ampelopsis
japonica
Amygdalus
persica purpurea
Andromeda
arborea

Azalea
altaclarense
pontica
Berberis
Thunbergii
vulgaris
- atropurpurea
Betula
purpurea
Cerasus
avium
Cornus
florida
Cotoneaster
Simonsii
Cratzous
prunifolia
Euonymus
europaus
- angustifolius
atropurpureus
latifolius
Koelreuteria
paniculata

\author{
Liquidambar \\ styraciflua \\ Mahonia \\ aquifolium \\ Parrotia \\ persica \\ Quercus \\ cocinnea \\ rubra \\ palustris \\ Rhus \\ cotinus \\ glabra \\ - laciniata \\ typhina \\ - laciniata \\ Ribes \\ missouriense \\ Stephanandra \\ flexuosa \\ Vaccinium \\ pensylvanicum \\ Viburnum \\ Opulus
}

\section{ORNAMENTAL FRUITING PLANTS.}

Arbutus
Unedo (Strawberry Tree)
Aucuba
japonica
Berberis
Darwinii
Thunbergii
Wilsona
Cotoneaster
frigida
microphylla
Simonsii
vulgaris
And others.
Cratæegus
Pyracantha
- Lxelandii

\section{Cydonia japonica \\ Elaxgnus longipes \\ Eugenia Ugni}

Euonymus europaus

Gaultheria procumbens Shallon

Hippophaë

rhamnoides (Sea Buckthorn)

8 Ilex (Holly), in variety

- Pernettya

8 b. mucronata

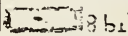

\section{Pyrus}

Aucuparia (Mountain Ash)

Malus, in variety

Sorbus

Rosa

rugosa

Ruscus

aculeatus (Butcher's Broom)

Sambucus

racemosa (Scarlet Berried Elder)

Skimmia japonica

Symphoricarpus racemosus (Snowberry)

Viburnum

Opulus (Guelder Rose) 
PLANTS FOR GROWING UNDER THE SHADE OF TREES, \&C., AND AS COVER FOR GAME.

Aucuba, of sorts

Berberis

Aquifolium

buxifolia

Darwinii

vulgaris

Black Thorn or Sloe

Box, of sorts

Brambles, of sorts

Broom

Butcher's Broom

(Ruscus)
Cotoneaster, of sorts

Daphne pontica

Euonymous japonicus

Holly, common

Hypericum or st. John's Wort

lvy, many sorts

Laurel, common

- Portugal

Periwinkle, of sorts

Phillyrea
Privet, Evergreen Box-leaved

Rhodode $n$ dron ponticum. This is generally considered to be the only plant that game never touch

Rosa rugosa

Snowberry (Symphori-

Sweet Briar

White Thorn

Yew, common

\section{TREES AND SHRUBS FOR PLANTING IN TOWNS.}

Acacia (Robinia)

Acer (Sycamore)

Ailanthus glandulosa

Almond

Aucuba

Berberis, in variety

Box

Broom (Cytisus)

Colutea

Cotoneaster

Deutzia

Dogwood (Cornus)

Elder (Sambucus)

Euonymus
Forsythia
Guelder Rose (Viburnum)
Hibiscus
Holly (small leaved)
Ivies (Hedera)
Laburnum
Laurels
Laurustinus
Lilacs (Syringa)
Limes
Oaks (evergreen)
Olearia Haastii,
Quotations on Application.

Euonymus

Forsythia

Guelder Rose (Viburnum) Hibiscus

Holly (small leaved)

Ivies (Hedera)

aburnum

Laurustinus

Lilacs (Syringa)

Limes

Olearia Haastii,

\section{AVENUE TREES.}
Osmanthus
Planes
Poplar, Canadian
Phillyrea
Privets
Pyracantha (Cratægus)
Rhododendron ponti
Ribes, in variety
Spiraeas, in variety
Sycamore
Thorns, in variety
Virginian Creeper
Weigela

cum

Planes, Limes, Elms, Chestnuts, Sycamores, American and Norway Maples, Walnuts, Ailanthus, Acacias, etc., cultivated specially for the above purpose, can be supplied in good strong well-rooted trees with straight stems, and symmetrical heads.

Quotations on application.

\section{HEDGE PLANTS.}

The following plants are admirably adapted for hedge planting. We shall be pleased to quote prices and submit samples of any upon application.

Arbor Vita, American
Lobl's Thuia
(Thuia gigantea)
Barberry (Berberis)
common
Darwin's
Box-leaved (dulcis)
Beech (Fagus)
common
purple
Black Thorn or Sloe
Box (Buxus), of sorts
Briar, Sweet
Broom
common yellow
white

Cherry Plum
(Prunus Myrobalana)
Cypress (Cupressus)
Lawson's
- erect
Nootkatensis
Euonymus
Furze (Ulex), double
Hazel (Corylus)
Holly (Ilex), common
green \& variegated
in variety
Hornbeam (Curpinus)
Laurel, common
myrtle-leaved
Portugal

Quotations on application.
Laurustinus, common

Lilac, of sorts

Privet (Ligustrum)

evergreen

golden oval-leaved

Quick or White Thorn

Rhododendron

ponticum

Snowberry

(Symphoricarpus)

Yew (Taxus), common

gold and silverstriped

upright English

Irish 


\section{TRANSPLANTED FOREST TREES.}

Acaciaor Locust Tree
Alder
Ash
Beech
Birch
Chestnut
Horse
Spanish
Elm
Chichester
Cornish
English
Huntingdon
Wych or Scotch
Fir
Hemlock
Silver
Spruce

Hazel

Holly
Hornbeam
Larch
common
Japanese
Lime
common
red-twigged
Maple, Norway
Mountain Ash
Oak
English
Turkey
American or Scarlet
Pine
Austrian
Hima I a an (Pinus
Corsican excelsa)
Pinaster (maritima)
Quotations on application.

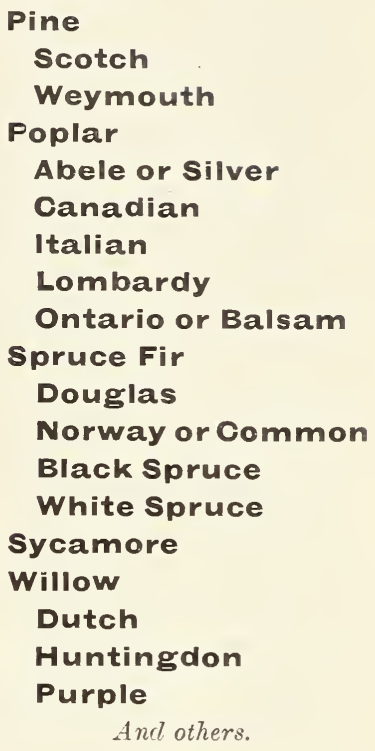

\section{PLANTS FOR THE BANKS AND MARGINS OF STREAMS.}

$\star$ (Those with an asterisk should be particlly submerged).

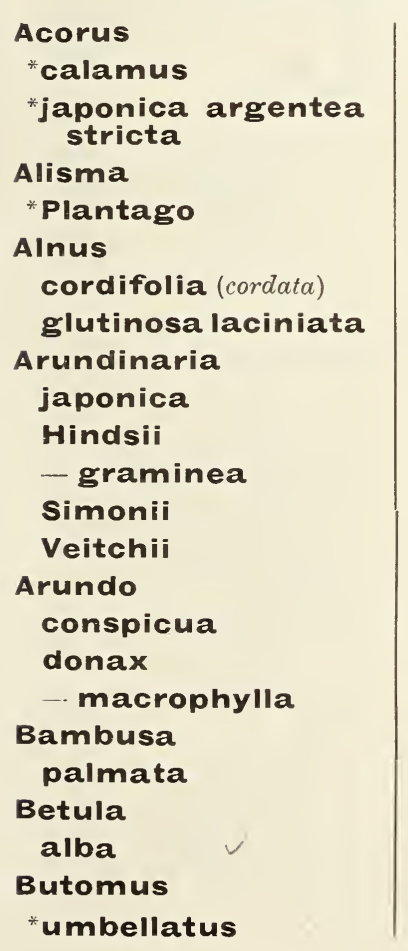

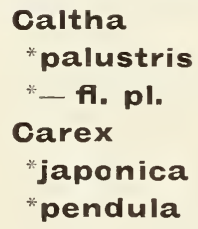

Caltha

*palustris

*-f. pl.

\section{Carex}

*japonica

* pendula

Cornus (Dogwood) alba Späthii sanguinea stolonifera

Cotoneaster microphylla

\section{Cyperus}

*alternifolius

*Iongus

Elymus glaucifolius

\section{Epilobium} angustifolium

hirsutum

album

\section{Erianthus}

*Ravenna

Eriophorum

*polystachyon

Quotutions on application.

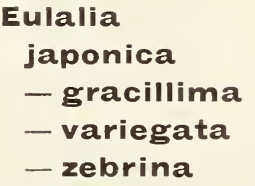

Eulalia japonica

- gracillima

- variegata

- zebrina

\section{Funkia}

japonica (grandiflora) lanceolata

Sieboldii

\section{Gunnera}

manicata

scabra

Gymnothrix

"latifolia

Gynerium argenteum

Hippophä rhamnoides

Iris

(see page 46)

Kæmpferi, named

- seedlings

ochroleuca

orientalis

* pseudo-acorus

[See overleaf. 
PLANTS FOR THE BANKS AND MARGINS OF STREAMS-continued.

Juncus
*effusus spiralis
Lysimachia
clethroides
Lythrum
roseum superbum
Myosotis
*palustris
semperflorens
Myrica
asplenifolia
Gale
Osmunda
regalis
Phyllostachys
aurea
Kumasasa
anceps
nigra
viridi-glaucescens

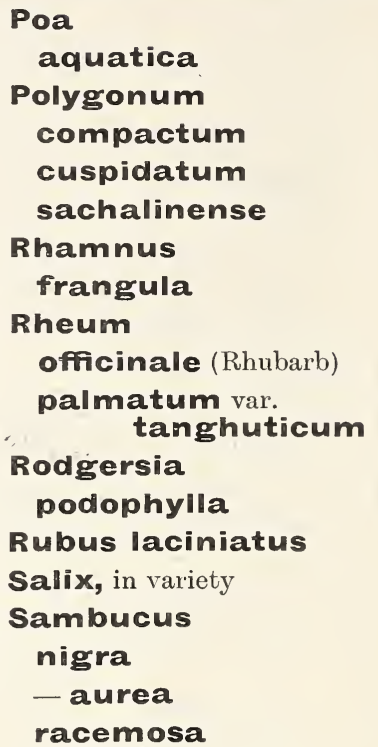

Poa

aquatica

Polygonum compactum cuspidatum sachalinense

Rhamnus frangula

Rheum officinale (Rhubarb) palmatum var. tanghuticum

Rodgersia podophylla

Rubus laciniatus

Salix, in variety

Sambucus

nigra

- aurea

racemosa

Sagittaria

*sagittafolia

Solidago multiradiata serotina

Sparganium

*ramosum

Spiraea aruncus

other varieties.

Stipa

pennata

Tamarix

Typha angustifolia latifolia

Trollius europaus.

Quotations on application.

TREES AND SHRUBS FOR SEA-SIDE PLANTING.

Abies sitchensis
Alder
Arbutus Unedo
Aristotelia Macqui
Ash, Mountain
Atriplex Halimus
Aucuba japonica
Beech
Box
Briar, Sweet
Broom
Cytisus
Buddleia globosa
Cassinia
fulvida
Choisya
ternata
Cotoneasters
Cupressus
macrocarpa
Elders

Elaagnus argentea
Elm
Wych
Guernsey
Escallonias
Euonymus
Fuchsia Riccartonii
Garrya elliptica
Griselinia littoralis
Guelder Rose
Holly, common
Hydrangea
Laurustinus
Leycesteria formosa
Lycium
Maples
Oak
Evergreen
Turkey
Osmanthus
Phillyreas

Pinus
austriaca
contorta
insignis (radiata)
Laricio
Pinaster
montana
Pittosporum
Poplars
Privets
Sea Buckthorn
Service Tree
Snowberry
Sweet Bay
Sycamore
Tamarisk
Thorns
Tsuga
Albertiana
canadensis
Veronicas
Willows.




\section{INDEX.}

\begin{tabular}{|c|c|c|c|c|c|}
\hline Abele (see Populus) & $\begin{array}{r}A G E \\
68\end{array}$ & Balsam Poplar (see & GE & Cassandra (see & AGE \\
\hline Abelia & 55 & Populus) 6 & 68 & Andromeda) & \\
\hline Abies & 5 & Bamboos & 43 & Cassinia (see & \\
\hline Abutilon & 55 & Bambusa (see also & & Diplopappus) & 36 \\
\hline Acacia (see Robinia) & 70 & Thamnocalamus), 4 & 43 & Castanea & 58 \\
\hline Acanthopanax (see Aralia) & 57 & and Arundinaria & 43 & Catalpa & 5 \\
\hline Acer & 55 & Barberry (see Berberis) & 57 & Cat's Tale (see Typha) & 90 \\
\hline Acorus & 90 & Bay (see Laurus) & 38 & Ceanothus & 87 \\
\hline Actinidia & 81 & Bear Oak (see Quercus) & 41 & Cedar (see Cedrus) & \\
\hline Egle (see Citrus) & 36 & Beech (see Fagus) & 64 & Cedar of Lebanon (see & \\
\hline Esculus & 56 & Benthamia (see also & 35 & Cedrus) & \\
\hline Ailanthus & $\begin{array}{l}56 \\
81\end{array}$ & 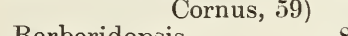 & & Cedrela & 5 \\
\hline $\begin{array}{l}\text { Akebia } \\
\text { Alaternus (see Rhamnus) }\end{array}$ & $\begin{array}{l}81 \\
39\end{array}$ & Berberidopsis & 81 & $\begin{array}{l}\text { Cedrus } \\
\text { Celastrus }\end{array}$ & \\
\hline Alaternus (see Rhamnus) & $\begin{array}{l}39 \\
56\end{array}$ & Berberis & 57 & $\begin{array}{l}\text { Celastrus } \\
\text { Cephalanthus }\end{array}$ & 8 \\
\hline $\begin{array}{l}\text { Alder (see Alnus) } \\
\text { Alexandrian Laurel (see }\end{array}$ & 56 & $\begin{array}{l}\text { Berchèmia } \\
\text { Betula }\end{array}$ & $\begin{array}{l}87 \\
57\end{array}$ & $\begin{array}{l}\text { Cephalanthus } \\
\text { Cephalotaxus }\end{array}$ & 3 \\
\hline $\begin{array}{l}\text { Alexanarian Laurel (see } \\
\text { Ruscus) }\end{array}$ & 39 & $\begin{array}{l}\text { Betula } \\
\text { Bignonia }\end{array}$ & $\begin{array}{l}57 \\
81\end{array}$ & Cerasus & \\
\hline Alisma & 90 & Biota & 7 & Cercidiphyllum & $5 ?$ \\
\hline All Saint's Cherry isee & & Birch (see Betula) & 57 & Cercis & 5 \\
\hline Cerasus) & 59 & Bird Cherry (see Cerasus) 5 & 59 & Chamæcyparis (see & \\
\hline Almond (see Amygdalus) & 56 & Bitter Almond (see & & Cupressus) & \\
\hline Alnus & 56 & Amygdalus) 5 & 56 & Cherry (see Cerasus) 36 , &, 5 \\
\hline Althæa (see Hibiscus) & 65 & Black Jack Oak (see & & Cherry Plum & 94 \\
\hline Amelanchier & 56 & Quercus) 4 & 41 & Chestnut & 9 \\
\hline American Allspice (see & & Black Spruce (see Abies) & 5 & Chile Pine (see Araucaria) & \\
\hline $\begin{array}{l}\text { Calycanthus } \\
\text { American and }\end{array}$ & 57 & $\begin{array}{l}\text { Black Thorn } \\
\text { Black Walnut (see }\end{array}$ & 94 & $\begin{array}{l}\text { Chimonanthus } \\
\text { Chinese Arbor Vitæ (see }\end{array}$ & 5 \\
\hline Japanese Plants & 28 & Juglans) 6 & 65 & $\begin{array}{r}\text { Biota) } \\
\text { B }\end{array}$ & \\
\hline American Arbor Vitæ (see & & Bladder Nut (see & & Chinese Yew (see & \\
\hline American Maple Thuia) & 14 & Staphylea) 7 & 72 & Cephalotaxus) & \\
\hline $\begin{array}{l}\text { American Maple } \\
\text { American Walnut (see }\end{array}$ & 94 & Bladder Senna (see & & Chionanthus & 5 \\
\hline $\begin{array}{r}\text { American Walnut (see } \\
\text { Carya) }\end{array}$ & & Colutea) 5 & 59 & Choisya & 3 \\
\hline Amorpha Carya) & 58 & Blue Spruce (see Abies) & 5 & Christ's Thorn (see & \\
\hline $\begin{array}{l}\text { Amorpha } \\
\text { Ampelopsis (see also }\end{array}$ & 56 & $\begin{array}{r}\text { Menyanthes) } 9 \\
\text { Dog Dedn (see }\end{array}$ & 90 & Cissus (see Vitis) & $\begin{array}{l}6 \\
8\end{array}$ \\
\hline Rhus, 70) & 81 & Box (see Buxus, 35) & & Cistus & 3 \\
\hline Amygdalopsis(see Prunus) & 68 & Bramble (see Rubus), & 70 & Citrus & \\
\hline Amygdalus & 56 & Brasenia & 90 & Cladrastis & 5 \\
\hline Andromeda & 28 & Bridgesia (see Ercilla) & 83 & Clematis & 8 \\
\hline Apera & 40 & Broom (see Cytisus) & 62 & Clerodendron & 5 \\
\hline Aponogeton & 90 & Broussonetia & 57 & Clethra & 2 \\
\hline Apple (see Pyrus) & 68 & Bruckenthalia & 28 & Cleyera & \\
\hline Aquatic Plants & 90 & Bryanthus & 28 & Climbers & \\
\hline Aralia & 57 & $\begin{array}{l}\text { Buddleia } \\
\text { Bupleurum }\end{array}$ & 57 & $\begin{array}{l}\text { Cluster Pine (see Pinus) } \\
\text { Cockspur Hawthorn (see }\end{array}$ & 1 \\
\hline Araucaria & 7 & Burr Oak (see Quercus) & 69 & Cratagus) & \\
\hline Arbor Vita (see Thuia), & 14 & Butcher's Broom (see & & Cocculus & \\
\hline Arbutus & 35 & Ruscus) 3 & 39 & Colletia & 3 \\
\hline Arctostaphylos & 35 & Butomus & 90 & Colutea & 5 \\
\hline Aristolochia & 81 & Button Wood (see & & Comptonia & 5 \\
\hline Aristotelia & 35 & Cephalanthus) 5 & 59 & Conifers & \\
\hline Aronia & 57 & Buxus & 35 & Corchorus (see Kerria) & 6 \\
\hline Arrhenatherum & 40 & Crsalpinia & 57 & Cork Oak (see Quercus) & 3 \\
\hline ArrowHead (seeSagittaria) & 90 & Californian Redwood (see & & Cornish Elm (see Ulmus) & 7 \\
\hline Artemisia & 57 & $\begin{array}{r}\text { Callornian Kedwood (see } \\
\text { Sequoia) } 1\end{array}$ & & $\begin{array}{l}\text { Cornus (see also } \\
\text { Benthamia } 35 \text { ) }\end{array}$ & \\
\hline Arundinaria (see also & & Calla & 90 & Benthamia, 35) & $\begin{array}{l}5 \\
2\end{array}$ \\
\hline Thamnocalamus, 43) & $\begin{array}{l}43 \\
40\end{array}$ & Calluna (see Erica) & 29 & $\begin{array}{l}\text { Corokea } \\
\text { Coronilla }\end{array}$ & \\
\hline Arundo & $\begin{array}{l}40 \\
64\end{array}$ & Calophaca & 57 & $\begin{array}{l}\text { Coronilla } \\
\text { Corsican Pine (see Pinus) }\end{array}$ & \\
\hline Ash (see Fraxinus) & $\begin{array}{l}64 \\
68\end{array}$ & Caltha & 90 & Cortaderia (see Gvnerium) & \\
\hline Aspen (see Populus) & $\begin{array}{r}68 \\
7\end{array}$ & Calycanthus & 57 & $\begin{array}{l}\text { Corylopsis } \\
\text { Cory Gynerium) }\end{array}$ & \\
\hline Athrotaxis & 81 & Camellia & 35 & $\begin{array}{l}\text { Corylopsis } \\
\text { Corylus }\end{array}$ & \\
\hline $\begin{array}{l}\text { Atragene (see Clematis) } \\
\text { Atriplex }\end{array}$ & $\begin{array}{l}81 \\
57\end{array}$ & Candleberry Myrtle (see & & $\begin{array}{l}\text { Corylus } \\
\text { Cotoneaster }\end{array}$ & \\
\hline $\begin{array}{l}\text { Atriplex } \\
\text { Aucuba }\end{array}$ & 35 & Myrica) 6 & 66 & Cranberry (see Oxycoccus) & \\
\hline Austrian Pine (see Pinus) & 9 & Caprifolium (see Lonicera) & & Cratægus 36 , & \\
\hline Autumn Tinted & & Caragana & $\begin{array}{l}84 \\
58\end{array}$ & $\begin{array}{l}\text { Crinodendron (see } \\
\text { Tricuspidaria) }\end{array}$ & \\
\hline Plants & 93 & Carex & & Cryptomeria & \\
\hline Azaleas & $\begin{array}{l}94 \\
24\end{array}$ & Carpentaria & 35 & Cunninghamia & \\
\hline Azara & 35 & $\begin{array}{l}\text { Carpinus } \\
\text { Carva }\end{array}$ & 58 & Cupressus (see also & \\
\hline Baccharis & 35 & Caryopteris & 58 & (5) & \\
\hline
\end{tabular}




Cyperus
Cypress (see Cupressus)
Cyrilla
Cytisus
Daboëcia (see Menziesia)
Dacrydium
Dactylis
Daphne
Daphniphyllum

Pyrus) 68
Date Plum (see Diospyros) 63

Davidia

Decaisnea

Deciduous Cypress (see Taxodium) ]3

\section{Deciduous Trees}

\section{and Shrubs}

Desfontainea

Desmodium

Deutzia

Diervilla

Digraphis (see Phalaris)

Dimorphanthus (see

Aralia) 57

Diospyros

Diplopappus

Dirca

Dogwood (see Cornus)

Double-flowering Peach (see Amygdalus) 56

Drimys 36

Dyer's Oak (see Quercus) 41

Edgeworthia

63

Edwardsia

Elæeagnus 37,64

Elder (see Sambucus) 71

Elm (see Ulmus) 73

Elymus 40

Embothrium $\quad 37$

Empetrum 29

English Elm (see Ulmus) 73

English Ivy (see Hedera) 83

English Oak (see Quercus) 69

English Yew (see Taxus) 13

Enkianthus 29

Epigea

Epilobium

Ercilla

Erianthus

Erica

Eriobotrya

Eriophorum

Escallonia

Eucalyptus

Eucommia

Eucryphia

Eugenia

Eulalia

Euonymus

Euptelea

Eurya

Eurybia (see Olearia)

Evergreen Trees and Shrubs

Evergreens for

Bedding 92

Evergreen Oak (see

Quercus) 39
Evergreen Plum (see Cerasus) 36

Evergreen Thorn (see Cratægus) 36

Exeter Oak (see Quercus) 41 Exochorda

Fabiana

Fagus

False Indigo (see

Amorpha) 56

Fetid Yew (see Torreya) 14

Fir

95

Flowering Ash (see

Fraxinus) 64

Flowering Rush (see

$$
\text { Butomus) } 90
$$

\section{Forest Trees}

95

Forget-me-not (see

Forsythia

Myosotis 90

Fothergilla

Fraxinus

Fremontia

Fringe Tree (see

Chionanthus) 59

Frog-bit (see Hydrocharis) 90

Fuchsia

64

Funereal Cypress (see

Cupressus) 8

Funkia

Furze (see Ulex)

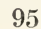

Garrya

Gaultheria

Gean (see Cerasus)

Genista

Giant Water Dock (see

Ginkgo

Rumex) 90

Glastonbury Thorn (see Cratægus) 62

Gleditschia

Glyceria 40,90

Glycine (see Wistaria) 85

Golden Arbor Vitæ (see

Thuia) 14

Golden Club(see Orontium) 90 Golden Larch (see Larix) 9 Golden Willow (see Salix) 70

Grape Vine (see Vitis) 85

Grevillea

Griselinia

Guelder Rose (see Viburnum)

Gunnera

Gymnocladus

Gymnothrix

Gynerium

Halesia

Halimodendron

Hamamelis

\section{Hardy Aquatic}

Plants 90

Hardy Heaths (see Erica) 29 Hawk's Foot Maple (see Acer) 56

Hawthorn (see Cratægus) 62 Hazel Nut (see Corylus) 61 Hedera

37,83

\section{Hedge Plants}

PAGE

Hedysarum

Helianthemum

emlock Spruce (see

Abies) 5

Hibiscus 65

Hickory (see Carya) 58

Himalayan Pine (see

Pinus) 11

Hippophaë

Holbœllia (see Stauntonia) 85

Holly (see Ilex)

Hollyleaved Oak (see

Quercus) 39

Honeysuckle (see

Lonicera) 85

Hop Tree (see Ptelea) 68

Hornbeam (see

Carpinus) 58

Horse Chestnut (see

$$
\text { Asculus) } 56
$$

Hottonia 90

Hungarian Oak (see Quercus) 41

Huntingdon Elm (see

Hydrangea (see also

$$
\text { Ulmus) } 73
$$

Schizophragma 89) 65

Hydrocharis

Hymenanthera

65
90

Hypericum

37,65

Idesia

Ilex (see also Othera)

Illicium

37

Incense Cedar (see

Libocedrus) 9

Indigofera $\quad 65$

Iris $\quad 90$

Irish Heath (see

Menziesia) 29

Irish Ivy (see Hedera) 83

Irish Juniper (see

Juniperus) 9

Irish Yew (see Taxus) 14

Itea $\quad 29$

Ivy (see Hedera) 83

Jamesia

65

Japanese Cedar (see

Cryptomeria) 7

Japanese Cherry (see Cerasus) 59

Japanese Quince (see Cydonia) 62

Jasminum 84

Judas Tree (see Cercis) $\quad 59$

Juglans 65

Juncus

Juniperus

Kalmia

Kerria

65

Kilmarnock Willow (see

Koelreuteria Salix) 70

Laburnum

Larch (see Larix)

Lardizabala

Laricopsis (see Larix)

Larix

Laurel (see Cerasus)

65

Laurus (see also Oreodaphne, 38) 38,65 
PAGE Larustinus (see Viburnum) 40 Lavandula 38

Lavender (see Lavandula) 38 Lead Plant (see Amorpha) 56 Leatherwood (see Dirca) 63

Ledum (see also

Leiophyllum Leiophyllum 29), 29

Lespedeza

Leucothoe

Leycesteria

Libocedrus

Ligustrum

Lilac (see Syringa)

Lime (see Tilia)

Limnocharis

Lindera

Liquidambar

Liriodendron 65

Locust Tree (see Robinia) 70

London Plane (see

\section{Platanus) 68}

Lonicera $36,66,84$

Loquat Tree (see

Loropetalum Eriobotrya) 87

Lucombe Oak (see Quercus) 41

Lycium

Lysimachia

Lythrum

Maclura

Magnolia

Mahonia (see Berberis) 38, 66

Maiden Hair Tree (see

Maple (see Acer) Ginkgo) 5

Marsh Marigold (see

Caltha) 90

Marsh Oak (see Quercus) 69

Menispermum 85

Menyanthes 90

Menziesia 29

Minorca Box (see Buxus) 35

Miscanthus (see Eulalia) 40

Mock Orange (see

Philadelphus) 67

Morus 66

Mountain Ash (see Pryus) 68 Mountain Pine (see Pinus) 11

Muehlenbeckia

Mulberry (see Morus) $\quad 66$

Myosotis

Myricaria (see Tamarix) 73

Myrica (see ulso Comptonia, 59)

66

Myrtus (see also

Nandina Eugenia, 37) 89

Neillia (see Spiræa) 72

Neviusia

Norway Maple (sce Acer) 56 Norway Spruce

Notospartium (see Abies)

Nut Pine (see Pinus) 11

Nuttallia

Oak (see Quercus) $\quad 39,69$

Olea

Olearia

Ononis
Oreodaphne

PAGE

38

Oriental Plane (see

Platanus) 68

Ornamental Fruiting Plants 93

Ornamental

Grasses 40

Ornus

66

Orontium

90

Osage Orange (see Maclura)66

Osmanthus

Osmunda

Osteomeles

Othera

Oxycoccus

Oxydendron

Ozothamnus

Pronia

Pailiurus

Parrotia

Passifiora (Passion Flower) 85

Paulownia 66

Pavia (see Asculus) 56

Peach (see Amygdalus) $\quad 56$

Pear (see Pryus) 68

Peltandra

Periploca

Periwinkle (see Vinca)

Pernettya

Perowskia

Persimmon (see Diospyros) 63

Phalaris

Philadelphus

Philesia

Phillyræa

Phlomis

Photinia

Phyllostachys

Picea (see Abies)

Pieris (see Andromeda)

Pine

Pin Oak (see Quercus)

Pinus

Piptanthus (see

Thermopsis) 89

Pitch Pine (see Pinus) 11

Pittosporum 39

Planera (see Zelkova) 74

Plane Tree(sce Platanus) $\quad 68$

Plants for Covering Buildings 87

Plants for margins of streams 95

Plants for shade or cover for Game 94

Platanus

Poa

68

Podocarpus

40,90

Poison Ivy (see Rhus) 70

Poison Oak (see Rhus) 70

Polygala

29

Polygonum

85,90

Pomegranate (see Punica) 89

Pontederia 90

Poplar (see Populus) 68

Populus

Portugal Laurel (see Cerasus)
Potentilla

PAGE

Prince Albert's Yew (see Saxe-Gothæa) 13

Prinos 39

Privet (see Ligustrum) 38

Prumnopitys 11

Prunus (see also Cerasus) 36,68

Pseudolarix (see Larix) 9

Pseudotsuga (see Abies) 5

Ptelea

Pterocarya

68

Pterostys

Punica (see Halesia) 64

Purple Beech (see Fagus) 64

Purple Willow (see Salix) 71

Pyracantha

94

Pyrus (see also Cydonia, 62) 68

Quercus $\quad 39,69$

Quick

Quince (see Cydonia) $\quad 62$

Ranunculus

Raphiolepis

Retinispora

Rhamnus

Rheum

Reed Mace (see Typha)

Rhodora

Rhodotypos

Rhus $\quad 70$

Ribes 70

Ringlet Willow (see Salix) 70

Robinia

Rock Cistus (see Helianthemum) 37

Rodgersia

96

Roman Cypress (see

Cupressus) $\mathrm{S}$

Rosa rugosa 94

Rose Acacia (see Robinia) 70

Rosemary (see Rosmarinus) 39

Rosmarinus $\quad 39$

Rubus

Rumex

Ruscus

Sagittaria

Salisburia (see Ginkgo) 8

Salt Tree (see

Halimodendron) 6

Salix 70

Sarcococea 39

Sambucus 71

Savin (see Juniperus) $\quad 9$

Saxe-Gothæa 13

Scarlet-berried Elder (see Sambucus) 93

Scarlet Maple (see Acer) 56

Scarlet Oak (see Quercus) 69

Scarlet Trumpet Honey suckle (see Lonicera) 85

Schizophragma $\quad 89$

Scarlet Willow (see Salix) 70

Sciadopitys $\quad 13$

Scirpus 90

Scotch Elm (see Ulmus) 73

Scotch Laburnum (see Laburnum) 65

Scotch Pine (see Pinus) 11 
Scrub Pine (see Pinus) PAGE Sea Buckthorn (see

\section{Hippophaë}

\section{Select Oaks}

Senecio

Sequoia

Service Tree (see Pyrus) 68

Shepherdia 7

Shrubby Honeysuckle (see Lonicera) 66

Siberian Crab (see Pyrus) 68

Silver Willow (see Salix) 71

Skimmia

Sloe (see Prunus)

Smilax

Snowball Tree (see

Snowberry (see

Viburnum) 74

Symphoricarpus) $\quad 72$

Snowdrop Tree(see Halesia) 64

Snowy Mespilus (see Amelanchier 56

Solanum

Solidago

Sophora

Southernwood (see

$$
\text { Artemisia) } 57
$$

Spanish Broom (see Spartium) 71

Spanish Chestnut (see Castanea) 58

Spanish Oak (see Quercus) 69 Sparganium

Spartium

Spindle Tree (see Euony-

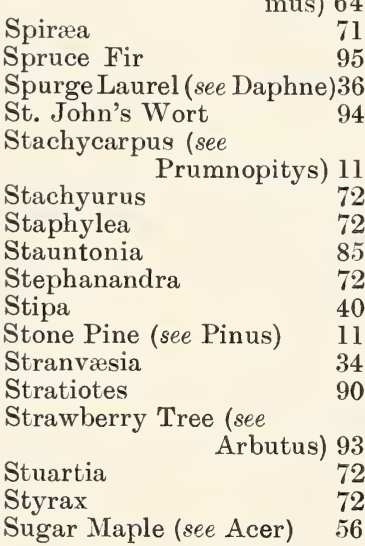

Sumach (see Rhus)

PAGE

Sweet Almond (see

Amygdalus) 56

Sweet Bay (see Laurus) $\quad 38$

Sweet Briar 94

Sweet Flag (see Acorus) 90

Sweet Gale (see Myrica) $\quad 66$

Sycamore (see Acer) $\quad 56$

Sycopsis $\quad 39$

Symphoricarpus $\quad 72$

Symplocos 72

Syringa

72

Tachamahac (see Populus) 68

Tamarix 73

Tamarisk (see Tamarix) 73

Tape Grass (seeVallisneria) 90

Tasmanian Cypress (see

Athrotaxis) 7

13

Taxus 13

Tecoma (see Bignonia) 81

Teucrium 89

Thamnocalamus $\quad 43$

Thermopsis 89

Thorns (see Cratægus) $36-62$

Thuia 14

Thuiopsis (see Cupressus, 8,

Tilia

Thuia) 14

Torreya

73

Traveller's Joy (see Clematis) 81

Tree Ivy (see Hedera)

37

Tree of Heaven (see

Ailanthus) 56

Tree Pæony (see Pæonia) 66

Trees and Shrubs for Seaside Planting 96

Trees and Shrubs for Towns 94

Tricuspidaria

Trochodendron $\quad 39$

39

Trollius $\quad 96$

True Service (see Pyrus) 68

Tsuga (see Aloies)

Tulip Tree (see

Liriodendron) 65

Turkey Oak (see Quercus) 69

Typha

90

Ulex

39

Ulmus

73

Umbrella Magnolia

(see Magnolia) 66
Umbrella Pine (see

PACE

Sciadopitys) 13

Vaccinium $\quad 29$

Vallisneria $\quad 90$

Venetian Sumach (see Rhus)

Veronica

40

Viburnum $\quad 40,74$

Villarsia $\quad 90$

Vinca $\quad 40$

Virgilia (see Cladrastis) $\quad 59$

Virginian Creeper (see

$$
\text { Ampelopsis) } 81
$$

Virgin's Bower (see

Vitex

Clematis) 81

Vitis (see also

Ampelopsis, 81)

Walnut (see Juglans) $\quad 65$

Water Arum (see Calla) $\quad 90$

Water Flag (see Iris) 90

Water Shield (see Brasenia) 90

Water Soldier (see

Water Violet (see

$$
\text { Stratiotes) } 90
$$

Hottonia) 90

Weeping Willow (see Salix)70

Weigela

Wellingtonia

Weymouth Pine(see Pinus) 11

White Beam Tree (see

Pyrus) 68

White Oak (see Quercus) 41

White Portugal Broom

White Thorn (see Cytisus) 62

Willow (see Salix) 70

Willow Oak (see Quercus) 70

Winter's Bark (see Drimys) 36

Wistaria

Witch Hazel (see

Wych Elm (see Ulmus) 65

Xanthoceras 74

Xanthorrhiza $\quad \mathbf{7 4}$

Yellow Broom (see Cytisus)62

Yellow Trumpet Honeysuckle (see Lonicera) 84

Yew (see Taxus)

13

Yucca

Yulan (see Magnolia)

Zelkova

Zenobia (see Adromeda) 28 
In addition to this Catalogue we publish the following Lists and Catalogues which we shall be happy to forward post free on application :-

VEGETABLE and FLOWER SEEDS, GARDEN TOOLS, SUNDRIES, \&C. INDOOR PLANTS, ORCHIDS, PALMS, FERNS, \&C.

hARDY herbaceous PERENNIALS and ALPINe PLANTS.

FRUIT TREES, GRAPE VINES, FIGS, \&C.

BULBS and FORCING PLANTS.

HARDY WATER LILIES.

NEW HARDY PLANTS FROM CHINA.

BEDding PLANTS.

ROSES.

CARNATIONS.

CHRYSANTHEMUMS.

DAHLIAS.

STRAWBERRIES.

JAMES VEITCH \& SONS, Ltd.,

Royal Exotic Nursery, CHElsea, LONDON. 
